\title{
Rational embeddings of hyperbolic groups
}

\author{
James Belk, Collin Bleak* and Francesco Matucci**
}

\begin{abstract}
We prove that all Gromov hyperbolic groups embed into the asynchronous rational group defined by Grigorchuk, Nekrashevych and Sushchanskil. The proof involves assigning a system of binary addresses to points in the Gromov boundary of a hyperbolic group $G$, and proving that elements of $G$ act on these addresses by asynchronous transducers. These addresses derive from a certain self-similar tree of subsets of $G$, whose boundary is naturally homeomorphic to the horofunction boundary of $G$.
\end{abstract}

Mathematics Subject Classification (2020). 20F65; 20F67, 20F10, 68Q70.

Keywords. Hyperbolic groups, rational group, Gromov boundary, horofunction boundary, transducers.

\section{Contents}

1 Background . . . . . . . . . . . . . . . . . 128

1.1 The rational group $R \ldots \ldots$. . . . . . . . . . . . . . . . . . . . . . . . . . 128

1.2 Hyperbolic groups . . . . . . . . . . . . . . . . 131

1.3 The horofunction boundary ................ 133

2 Rational groups for self-similar trees . . . . . . . . . . . . . . . . . . . . . . . . . 137

2.1 Self-similar trees . . . . . . . . . . . . . . . . 138

2.2 Rational homeomorphisms ................. 141

2.3 Rigid structures ... . . . . . . . . . . . . . . . . . 147

2.4 Rigid trees and $R_{2} \ldots \ldots \ldots \ldots$. . . . . . . . . . . 149

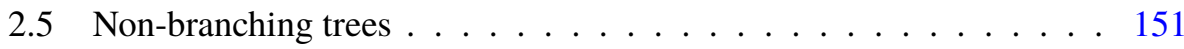

3 Rational actions of hyperbolic groups . . . . . . . . . . . . . . . 156

* The first and second authors have been partially supported by EPSRC grant EP/R032866/1 during the creation of this paper.

** The third author is a member of the Gruppo Nazionale per le Strutture Algebriche, Geometriche e le loro Applicazioni (GNSAGA) of the Istituto Nazionale di Alta Matematica (INdAM) and gratefully acknowledges the support of the Fundação de Amparo à Pesquisa do Estado de São Paulo (FAPESP Jovens Pesquisadores em Centros Emergentes grant 2016/12196-5), of the Conselho Nacional de Desenvolvimento Científico e Tecnológico (CNPq Bolsa de Produtividade em Pesquisa PQ-2 grant 306614/2016-2) and of the Fundação para a Ciência e a Tecnologia (CEMAT-Ciências FCT projects UIDB/04621/2020 and UIDP/04621/2020). 
3.1 The tree of atoms . . . . . . . . . . . . . . . 157

3.2 Infinite atoms and the horofunction boundary $\ldots \ldots \ldots \ldots 161$

3.3 Nearest neighbors and visibility . . . . . . . . . . . . . . . . 162

3.4 A membership test for atoms . . . . . . . . . . . . . . . 165

3.5 Finitely many types . . . . . . . . . . . . . . . . 168

3.6 Proof of rationality . . . . . . . . . . . . . . . . . 171

4 An example . . . . . . . . . . . . . . . . . . . . . . 174

4.1 The atoms . . . . . . . . . . . . . . . . . . . . . . . . . . . . 174

4.2 The group . . . . . . . . . . . . . . . . . . . . 178

References . . . . . . . . . . . . . . . . . . . . . . 180

\section{Introduction}

Let $\{0,1\}^{\omega}$ denote the Cantor set of all infinite binary sequences. A homeomorphism of $\{0,1\}^{\omega}$ is said to be rational if there exists an asynchronous transducer (i.e. an asynchronous Mealy machine) that implements the homeomorphism on infinite binary strings. In [24], Grigorchuk, Nekrashevych and Sushchanskil observe that the set of all rational homeomorphisms of $\{0,1\}^{\omega}$ forms a group $\mathbb{R}$ under composition, which they refer to as the rational group. They also observe that the group of rational homeomorphisms of $A^{\omega}$ is isomorphic to $\mathbb{R}$ for any finite alphabet $A$ with at least two elements.

Here the word asynchronous refers to transducers that can output a finite binary sequence of any length each time they take a digit as input. This is a generalization of synchronous transducers, which are required to output a single binary digit each time they take a digit of input. The asynchronous rational group $R$ contains the group of synchronous rational homeomorphisms corresponding to any finite alphabet.

Our main focus is on embedding questions for the rational group $R$. We prove:

Theorem 1. Every hyperbolic group embeds into $\mathbb{R}$.

Here a hyperbolic group is a finitely generated group $G$ whose Cayley graph satisfies Gromov's thin triangles condition (see [10]). This is a vast class of finitely presented groups: in a precise sense, "generic" finitely presented groups are hyperbolic [13,42].

There are compelling practical features of groups realised as groups of homeomorphisms of Cantor spaces realisable as finite-state transducers. For example, one can directly understand how such group elements act on their respective Cantor spaces and one can study the specific combinatorics of the transducers representing these group elements. An example of the impact that can be created by realising group elements this way is provided by Grigorchuk and Zuk. By realising the lamplighter group as a group of synchronous automata, they are able to compute the spectrum of the resulting group [25]. This yields a counterexample to a strong form of Atiyah's 
conjecture about the range of values of the spectrum of $L^{2}$-Betti numbers for closed manifolds [23].

If we consider the case of the groups $\operatorname{Aut}\left(\{0,1, \ldots, n-1\}^{\mathbb{Z}}, \sigma\right)$ of automorphisms of (full) shift spaces, then Grigorchuk, Nekrashevych, and Suschanskiǐ in [24] answer a request by Kitchens for a new combinatorial realisation of elements of those automorphism groups. Kitchens in [32] states that a major obstacle in the progression of understanding groups of automorphisms of shift spaces has been a lack of a practical combinatorial description for elements of these groups. Grigorchuk, Nekrashevych, and Suschanskiı̌ give an embedding of $\operatorname{Aut}\left(\{0,1, \ldots, n-1\}^{\mathbb{Z}}, \sigma\right)$ in $R$. There is now a second realisation arising from the recent description of the group $\operatorname{Aut}\left(G_{n, r}\right)$ of automorphisms of the Higman-Thompson group $G_{n, r}$ (for $1 \leq r<n$ natural numbers) as a group of transducers acting on a specific Cantor space $\mathfrak{C}_{n, r}$ [8], which also exposed an unexpected connection between subgroups of the outer automorphism group of the Higman-Thompson group $G_{n, r}$ and $\operatorname{Aut}\left(\{0,1, \ldots, n-1\}^{\mathbb{Z}}, \sigma\right)$. This connection arose through the study in $[8,9]$ of the special combinatorial properties of the transducers representing the group elements of $\operatorname{Aut}\left(G_{n, r}\right)$ for such $n$ and $r$, and leads to an explicit combinatorial realisation of elements of $\operatorname{Aut}\left(\{0,1, \ldots, n-1\}^{\mathbb{Z}}, \sigma\right)$ which exposes these groups' structures as non-split extensions over a central $\mathbb{Z}$.

Groups of synchronous transducers have received much attention in the literature, primarily as this class of groups contain numerous 'exotic' groups providing examples of unusual or unexpected behaviour (e.g. [4, 21, 22, 27, 39, 40] provides a very incomplete list of papers). While these groups do provide counterexamples to various forms of the Burnside conjecture and Milnor's conjecture, they also remain natural in many ways. Indeed, this class houses well known foundational groups which arise in other circumstances, including free groups [45], $\mathrm{GL}_{n}(\mathbb{Z})$ and its subgroups [11], the solvable Baumslag-Solitar groups $B S(1, m)$ [5], and the generalized lamplighter groups $(\mathbb{Z} / n \mathbb{Z})<\mathbb{Z}[44]$.

On the other hand, comparatively little attention has been paid to the more complex class of groups generated by asynchronous transducers, and the full asynchronous rational group $\mathbb{R}$ of Grigorchuk, Nekrashevych, and Sushchanskir. It is known that $R$ is simple and not finitely generated [7]. Also, while the word problem is solvable in finitely generated subgroups of $\mathbb{R}$ [24], the periodicity problem for elements of $\mathbb{R}$ has no solution [6]. Finally, the group $R$ houses 'exotic' groups of another type: the $\mathrm{R}$. Thompson groups $F, T$, and $V$ all embed into $R$ [24], as do the Brin-Thompson groups $n V$ (see [6] for the embedding of the group $2 V$ ) and groups such as the Röver group $V_{\Gamma}$ (a finitely presented simple group which is marriage of Grigorchuk's group $\Gamma$ with the R. Thompson group $V$, see [43]). Any group of synchronous automata embeds into $R$, so $R$ also contains the groups mentioned earlier.

The proof of Theorem 1 is dynamical as opposed to algebraic. Indeed, there is a general dynamical procedure for showing that a group embeds into $R$, which can be defined as follows. 
Definition 2. Let $G$ be a group acting by homeomorphisms on a compact metrizable space $X$. We say that the action of $G$ on $X$ is rational if there exists a quotient map $q:\{0,1\}^{\omega} \rightarrow X$ and a homomorphism $\varphi: G \rightarrow \mathbb{R}$ such that the diagram

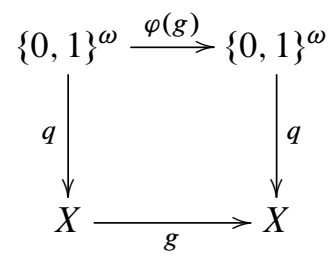

commutes for all $g \in G$.

Note that every compact metrizable space is a quotient of the Cantor set $\{0,1\}^{\omega}$, so it makes sense to ask whether any action of a countable group on such a space is rational. A group $G$ that acts faithfully and rationally on a compact metrizable space must embed into $R$. The converse holds as well, since any subgroup of $\mathbb{R}$ acts faithfully and rationally on the Cantor set.

Now, every hyperbolic group $G$ has a Gromov boundary $\partial G$, which is a compact metrizable space (see [26], and more generally the survey [30]) on which $G$ acts by homeomorphisms. Our main theorem is the following:

Theorem 3. For any hyperbolic group $G$, the action of $G$ on $\partial G$ is rational.

This theorem can be generalized as follows.

Corollary 4. Let $X$ be a geodesic, hyperbolic metric space, and let $G$ be a group acting properly and cocompactly by isometries on $X$. Then the action of $G$ on $\partial X$ is rational.

Proof. By the Švarc-Milnor lemma [10, Proposition I.8.19], we know that $X$ is quasi-isometric to $G$. It follows that $G$ is hyperbolic, and there exists a $G$-equivariant homeomorphism from $\partial X$ to $\partial G$ [10, Propositions III.1.9 and III.1.10].

These statements can be viewed as assigning a certain kind of symbolic dynamics to the action of the group $G$ on $\partial G$ (or $\partial X$ ). Specifically, the quotient map $q:\{0,1\}^{\omega} \rightarrow \partial G$ assigns one or more binary addressed to each point of $\partial G$, and elements of $G$ act on $\partial G$ by asynchronous transducers. In fact, our binary coding assigns exactly one binary address to each point in the well-known horofunction boundary (or metric boundary) $\partial_{h} \Gamma$ of $\Gamma$ (see, e.g. [2, 17, 29,33,37,46-48]). The horofunction boundary is compact and totally disconnected, and has the Gromov boundary $\partial \Gamma$ as a quotient [48]. Our construction computes the horofunction boundary of $\Gamma$ explicitly, and describes the action of $G$ on $\partial_{h} \Gamma$ by asynchronous transducers.

Symbolic dynamics for actions of hyperbolic groups on their boundaries have been studied extensively. For example, following a suggestion of Gromov, Coornaert 
and Papadopoulos prove that a certain space $\Phi_{0}$ related to the horofunction boundary (see Remark 1.31) is a subshift of finite type [14], and use this to give a symbolic coding for the associated geodesic flow. Though our assignment of binary addresses is similar in spirit to theirs, there does not seem to be a direct translation between them, and so it is unclear whether the natural action of $G$ on $\Phi_{0}$ is rational.

It follows immediately from Theorem 3 that any hyperbolic group $G$ that acts faithfully on $\partial G$ embeds into $R$. Unfortunately, it is possible for the action of $G$ on $\partial G$ to have nontrivial kernel, which is always a finite normal subgroup of $G$ as long as $G$ is non-elementary (see Proposition 1.18 below). However, as long as $G$ is nontrivial, it is easy to show that the free product $G * \mathbb{Z}$ is a non-elementary hyperbolic group with no finite normal subgroups. It follows that $G * \mathbb{Z}$ embeds into $R$, and hence $G$ does as well, which proves Theorem 1 from Theorem 3 .

Our proof of Theorem 3 begins by defining a very broad class of trees which have a notion of rational homeomorphisms on their boundaries. We refer to these as selfsimilar trees, and we prove in Section 2 that the group of rational homeomorphisms of the boundary of a self-similar tree acts on the boundary in a rational way in the sense of Definition 2. This seems to be a very general tool for proving that actions are rational, and we hope that it will be helpful in other contexts.

Next we define a tree of subsets of any hyperbolic graph $\Gamma$, which we refer to as atoms. Assuming a group $G$ acts properly and cocompactly on $\Gamma$, we prove in Section 3 that this tree is self-similar and its boundary is naturally homeomorphic to the well-known horofunction boundary (or metric boundary) $\partial_{h} \Gamma$ of $\Gamma$. The horofunction boundary is compact and totally disconnected, and has the Gromov boundary $\partial \Gamma$ as a quotient [48]. Our construction computes the horofunction boundary of $\Gamma$ explicitly, and describes the action of $G$ on $\partial_{h} \Gamma$ by asynchronous transducers. See Section 4 for an example of this construction.

Perhaps as evidence of the naturality or importance of the general construction, we learned from the authors that the article [34] gives a similar construction, with the goal of extending self-similar groups to act on the path space of a graph. While the constructions given for the self-similar trees (in their language, self-similar groupoids) are similar, the automata groups arising in [34] are quite different in nature from ours (e.g. they are synchronous).

The transducers that arise in our construction appear to have a special flavour: in all examples that we have computed, they act as prefix-exchange maps on a dense open subset of the boundary. As such, the embeddings we construct are "almost" embeddings of hyperbolic groups into Thompson's group $V$. In [35], Lehnert and Schweitzer use a push-down automaton that implements prefix exchanges on a finite set of test points to prove that all finitely-generated subgroups of Thompson's group $V$ are in the class of CoCF groups introduced by Holt, Röver, Rees, and Thomas [28]. If this method can be extended, it may be possible to use our embedding to shed some light on the question of whether hyperbolic groups are CoCF. 
Finally, note that while synchronous automata groups are always residually finite, the same does not hold true for asynchronous automata groups. In particular, our result does not yield any immediate information about the question of whether all hyperbolic groups are residually finite (see [19,31,36,41]).

Acknowledgements. We would like to thank Michael Whittaker for discussions where we learned that our papers have somewhat similar constructions of the selfsimilar tree and Volodymyr Nekrashevych for interesting discussions about our results in general.

\section{Background}

1.1. The rational group $R$. In this section we briefly recall the definitions of transducers and rational homeomorphisms from [24]. We have modified some of the definitions slightly to simplify the terminology.

Throughout this paper, if $S$ is a set, we let $S^{\omega}$ denote the set of all infinite sequences of elements of $S$, and we let $S^{*}$ denote the set of all finite sequences of elements of $S$, including the empty sequence $\varepsilon$.

Definition 1.1. A transducer consists of the following data:

(1) Two finite sets $A_{\text {in }}$ and $A_{\text {out }}$ called the input alphabet and output alphabet.

(2) A finite set $Q$ whose elements are called states.

(3) An initial state $q_{0} \in Q$.

(4) A transition function $t: Q \times A_{\text {in }} \rightarrow Q$.

(5) An output function $o: Q \times A_{\text {in }} \rightarrow A_{\text {out }}^{*}$.

A transducer is synchronous if $o(q, a)$ is a single symbol in $A_{\text {out }}$ for each $q \in Q$ and $a \in A_{\text {in }}$, and asynchronous otherwise. We allow both synchronous and asynchronous transducers.

We can draw a transducer as a finite directed graph, as shown in Figure 1. Each state of this transducer is a node of the graph, and the directed edges indicate the transitions and output. Specifically, for each $q \in Q$ and $a \in A_{\text {in }}$, there is a directed edge in from $q$ to $t(q, a)$ in the graph with label $a \mid o(q, a)$.

If $T=\left(A_{\text {in }}, A_{\text {out }}, Q, q_{0}, t, o\right)$ is a transducer, an input string for $T$ is any infinite string $a_{1} a_{2} \cdots \in A_{\text {in }}^{\omega}$. The corresponding output string is the concatenation

$$
o\left(q_{0}, a_{1}\right) o\left(q_{1}, a_{2}\right) o\left(q_{2}, a_{3}\right) \cdots,
$$

where $\left\{q_{n}\right\}$ is the sequence of states starting at the initial state $q_{0}$ defined recursively by $q_{n}=t\left(q_{n-1}, a_{n}\right)$.

Note that the output string may be finite if $o\left(q_{n-1}, a_{n}\right)=\varepsilon$ for all but finitely many $n$, but we are interested in transducers whose output strings are always infinite. 


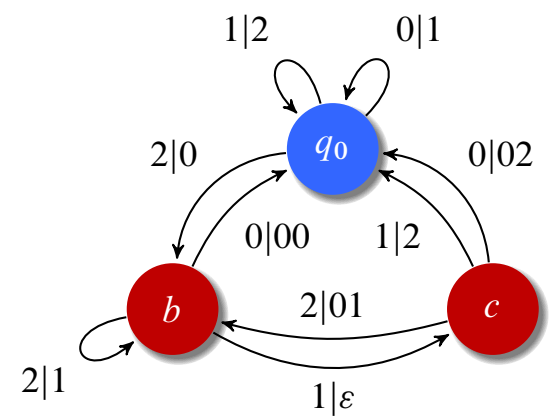

Figure 1. An asynchronous transducer on a three-letter alphabet $\{0,1,2\}$ with initial state $q_{0}$.

Such transducers are called nondegenerate. A nondegenerate transducer defines a function $A_{\mathrm{in}}^{\omega} \rightarrow A_{\mathrm{out}}^{\omega}$ from infinite input strings to infinite output strings.

Definition 1.2. Let $A_{\text {in }}$ and $A_{\text {out }}$ be finite sets. We say that a function $f: A_{\text {in }}^{\omega} \rightarrow A_{\text {out }}^{\omega}$ is rational if there exists a nondegenerate transducer with input alphabet $A_{\text {in }}$ and output alphabet $A_{\text {out }}$ whose output string is $f(\psi)$ for each input string $\psi \in A_{\text {in }}^{\omega}$.

The following properties of rational functions are proven in [24]. We will prove (2) and (3) in a more general setting in Section 2.2.

\section{Proposition 1.3.}

(1) Any rational function $f: A^{\omega} \rightarrow B^{\omega}$ is continuous with respect to the product topologies on $A^{\omega}$ and $B^{\omega}$.

(2) If $f: A^{\omega} \rightarrow B^{\omega}$ and $g: B^{\omega} \rightarrow C^{\omega}$ are rational, then so is the composition $g \circ f$.

(3) If $f: A^{\omega} \rightarrow B^{\omega}$ is a rational bijection, then the inverse $f^{-1}: B^{\omega} \rightarrow A^{\omega}$ is rational.

Definition 1.4. If $A$ is a finite set with at least two elements, the rational group $R_{A}$ is the group of all rational homeomorphisms of $A^{\omega}$.

In particular, the binary rational group $\mathbb{R}_{2}$ is the group of all rational homeomorphisms of the Cantor set $\{0,1\}^{\omega}$.

Proposition 1.5. For any two finite sets $A$ and $B$ with at least two elements, there exists a rational homeomorphism $A^{\omega} \rightarrow B^{\omega}$, and therefore the rational groups $\mathbb{R}_{A}$ and $R_{B}$ are isomorphic.

Proof. See [24, Corollary 2.12].

Thus up to isomorphism there is only one rational group $\mathbb{R}$, whose simplest form is the binary rational group $R_{2}$. Other rational groups $R_{A}$ are just other manifestations of this group. We will henceforth use the notation $R$ for the rational group in cases where the alphabet is unimportant. 
Note 1.6. In fact, Proposition 1.5 tells us that the rational groups $\mathbb{R}_{A}$ and $\mathbb{R}_{B}$ corresponding to two alphabets $A$ and $B$ are actually conjugate, in the sense that the action of $R_{A}$ on $A^{\omega}$ is conjugate to the action of $R_{B}$ on $B^{\omega}$ by a homeomorphism $A^{\omega} \rightarrow B^{\omega}$. Being conjugate is the natural geometric notion of equivalence for groups of homeomorphisms, and is stronger than saying that the two groups are algebraically isomorphic.

It follows from this conjugacy that the definition of a rational action given in Definition 2 does not depend on the alphabet. That is, if $G$ is any group acting on a compact metrizable space $X$ and there exists a finite alphabet $A$, a quotient map $q: A^{\omega} \rightarrow X$, and a homomorphism $\varphi: G \rightarrow \mathbb{R}_{A}$ such that $q \circ \varphi(g)=g \circ q$ for all $g \in G$, then the action of $G$ on $X$ is rational.

When working with a rational group $\mathbb{R}_{A}$, it often helps to consider the infinite directed tree $A^{*}$ of all finite strings over $A$. The root of $A^{*}$ is the empty string $\varepsilon$, and there is an edge from a string $w_{1}$ to another string $w_{2}$ whenever $w_{2}=w_{1} a$ for some letter $a \in A$. The Gromov boundary $\partial A^{*}$ of $A^{*}$ is naturally homeomorphic to $A^{\omega}$.

If $\alpha \in A^{*}$ is a finite string, we will let $A_{\alpha}^{*}$ denote the rooted subtree of $A^{*}$ with root $\alpha$, i.e. the set of all finite strings that have $\alpha$ as a prefix. The boundary $\partial A_{\alpha}^{*}$ is naturally a subset of $A^{\omega}$, consisting of all infinite strings in $A^{\omega}$ that have $\alpha$ as a prefix.

If $S \subseteq A^{\omega}$ is nonempty, the greatest common prefix of $S$ is the longest string $\alpha$ that is a prefix of all strings in $S$. If $S$ has at least two points then $\alpha$ must be a finite string. In this case, $\alpha$ is the deepest vertex (i.e. farthest vertex from the root) in $A^{*}$ with the property that $S \subseteq \partial A_{\alpha}^{*}$.

Definition 1.7. Let $f: A^{\omega} \rightarrow B^{\omega}$ be a rational map. Let $\alpha \in A^{*}$, and suppose that $f\left(\partial A_{\alpha}^{*}\right)$ has at least two points. Then the restriction of $f$ to $\alpha$ is the function $\left.f\right|_{\alpha}: A^{\omega} \rightarrow B^{\omega}$ defined by

$$
f(\alpha \psi)=\left.\beta f\right|_{\alpha}(\psi)
$$

for all $\psi \in A^{\omega}$, where $\beta$ denotes the greatest common prefix of $f\left(\partial A_{\alpha}^{*}\right)$.

There is a useful characterization of rational functions based on their restrictions.

Theorem 1.8. Let $A$ and $B$ be finite sets and let $f: A^{\omega} \rightarrow B^{\omega}$ be a continuous function. Then $f$ is rational if and only if the following conditions are satisfied:

(1) $f$ has only finitely many different restrictions.

(2) For each $\alpha \in A^{*}$ such that $f\left(\partial A_{\alpha}^{*}\right)$ is a one-point set $\{\psi\}$, the string $\psi \in B^{\omega}$ is eventually periodic.

Proof. See [24, Theorem 2.5].

We will use this theorem in Section 2.2 to generalize the notion of rational functions to the boundary of arbitrary self-similar trees. 
1.2. Hyperbolic groups. In this section we briefly recall relevant facts about hyperbolic graphs and hyperbolic groups.

If $\Gamma$ is a connected graph, a path in $\Gamma$ is a sequence $v_{0}, v_{1}, \ldots, v_{n}$ of vertices such that $v_{i-1}$ and $v_{i}$ are connected by an edge for all $1 \leq i \leq n$. The number $n$ is called the length of the path. A path between two vertices $v$ and $w$ is called a geodesic if it has the minimum possible length, and the length of such a path is the distance between $v$ and $w$, denoted $d(v, w)$. This notion of distance defines a metric on the vertex set of $\Gamma$, sometimes called the path metric.

Throughout this paper, we will regard a graph $\Gamma$ as being the same as its vertex set endowed with the path metric. In particular, we will write $v \in \Gamma$ to mean that $v$ is a vertex of $\Gamma$. Note that two such graphs are isomorphic if and only if they are isometric.

For the following definition, a geodesic triangle in $\Gamma$ with vertices $v_{1}, v_{2}, v_{3}$ is a triple $\left(\left[v_{1}, v_{2}\right],\left[v_{1}, v_{3}\right],\left[v_{2}, v_{3}\right]\right)$, where each $\left[v_{i}, v_{j}\right]$ is a geodesic from $v_{i}$ to $v_{j}$.

Definition 1.9. Let $\delta \geq 0$. A connected graph $\Gamma$ is $\boldsymbol{\delta}$-hyperbolic if for every geodesic triangle $([a, b],[a, c],[b, c])$ in $\Gamma$ and every vertex $v \in[a, b]$, there exists a vertex $w \in[a, c] \cup[b, c]$ so that $d(v, w) \leq \delta$.

We say that a graph $\Gamma$ is hyperbolic if it is $\delta$-hyperbolic for some $\delta \geq 0$. This definition is due to Gromov [26], and can be generalized to arbitrary metric spaces. See [10] for a general introduction.

There is a natural notion of boundary for a hyperbolic graph, also introduced by Gromov. If $\Gamma$ is a connected graph, a geodesic ray in $\Gamma$ is a sequence $\left\{v_{n}\right\}_{n \geq 0}$ of vertices such that each initial subpath $v_{0}, \ldots, v_{n}$ is a geodesic. Two geodesic rays $R=\left\{v_{n}\right\}$ and $R^{\prime}=\left\{v_{n}^{\prime}\right\}$ are said to fellow travel if the sequence $\left\{d\left(v_{n}, v_{n}^{\prime}\right)\right\}$ of distances is bounded. This is clearly an equivalence relation on geodesic rays, and we denote the equivalence class of a geodesic ray $R$ by $[R]$.

Definition 1.10. The Gromov boundary of $\Gamma$ is the set

$$
\partial \Gamma=\{[R] \mid R \text { is a geodesic ray in } \Gamma\} .
$$

Assuming the graph $\Gamma$ is locally finite, there is a natural topology on $\partial \Gamma$ which gives it the structure of a compact metrizable space (see [10]). We will henceforth assume that all graphs under consideration are locally finite.

Example 1.11. Any tree $T$ is 0 -hyperbolic, for if $([a, b],[a, c],[b, c])$ is a geodesic triangle in $T$ then $[a, b] \subseteq[a, c] \cup[b, c]$.

If we fix a point $p \in T$, then every infinite path of distinct vertices starting at $p$ is a geodesic ray. No two such rays fellow travel, and the Gromov boundary $\partial T$ can be identified with the set of all such rays.

Example 1.12. Any graph that is quasi-isometric to the hyperbolic plane $\mathbb{H}^{2}$ is hyperbolic. For example, the 1-skeleton of any tiling of $\mathbb{H}^{2}$ by congruent polygons is hyperbolic. The Gromov boundary of such a graph is homeomorphic to a circle, namely the circle of boundary points for the hyperbolic plane. 


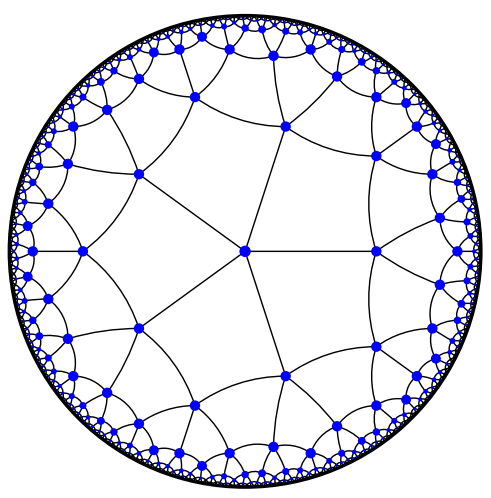

Figure 2. The 1-skeleton of the order five square tiling of the hyperbolic plane.

One such graph is shown in Figure 2. This is the 1-skeleton of the order five square tiling of the hyperbolic plane, i.e. the tiling by congruent regular quadrilaterals (hyperbolic squares) with five squares meeting at every vertex. We will be using this hyperbolic graph throughout this paper to illustrate our definitions and methods.

An isomorphism between two hyperbolic graphs induces a homeomorphism between their boundaries, and in particular any automorphism of a hyperbolic graph $\Gamma$ induces a self-homeomorphism of $\partial \Gamma$. Thus any action of a group $G$ on $\Gamma$ by isometries induces an action of $G$ on $\partial \Gamma$ by homeomorphisms.

More generally, if $\Gamma$ and $\Gamma^{\prime}$ are quasi-isometric graphs, then $\Gamma$ is hyperbolic if and only if $\Gamma^{\prime}$ is hyperbolic, in which case $\partial \Gamma$ is homeomorphic to $\partial \Gamma^{\prime}$.

Definition 1.13. A hyperbolic group is a finitely generated group whose Cayley graph is hyperbolic.

Of course, a finitely-generated group has many possible Cayley graphs, corresponding to the different finite generating sets. However, any two such Cayley graphs are quasi-isometric, and hence they are all hyperbolic if any one of them is hyperbolic. Moreover, the homeomorphism type of the boundary does not depend on the generating set, so it makes sense to talk about the Gromov boundary $\partial G$ of a hyperbolic group as a compact metrizable space.

Example 1.14. Any finite group or more generally any virtually cyclic group is hyperbolic. These are the elementary hyperbolic groups.

Example 1.15. Any finitely generated free group is hyperbolic, since the corresponding Cayley graph is a tree. More generally, any virtually free group, such as any free product of finite groups, is hyperbolic.

Example 1.16. A hyperbolic surface group is the fundamental group of a closed surface with Euler characteristic $\chi<0$. Such a group is hyperbolic, since its Cayley graph can be viewed as the 1-skeleton of a tiling of the hyperbolic plane $\mathbb{H}^{2}$ by congruent $(4-2 \chi)$-gons. 
Example 1.17. Let $\Gamma$ be a hyperbolic graph, and let $G$ be a group acting on $\Gamma$ by isometries. We say that the action of $G$ on $\Gamma$ is proper if every vertex in $\Gamma$ has finite stabilizer, and cocompact if there are finitely many orbits of vertices in $\Gamma$. If the action of $G$ is proper and cocompact, then the Cayley graph of $G$ must be quasi-isometric to $\Gamma$, and therefore $G$ is hyperbolic. For example, the isometry group of the 1-skeleton of the order five square tiling of the hyperbolic plane is hyperbolic (see Example 1.12).

We do need a few nontrivial facts from the theory of hyperbolic groups. The first is the following proposition, which we used in the introduction to prove Theorem 1 from Theorem 3.

Proposition 1.18. Let $G$ be a non-elementary hyperbolic group. Then the kernel of the action of $G$ on $\partial G$ is a finite normal subgroup of $G$.

Proof. Since $G$ is non-elementary, the Gromov boundary $\partial G$ is infinite (see [30, Theorem 2.28]). Any element of infinite order has exactly two fixed points in $\partial G$ (see [30, Proposition 4.2(1)]), so no infinite order element is contained in the kernel $K$ of the action of $G$ on $\partial G$. But every torsion subgroup of a hyperbolic group must be finite (see [20, Corollaire 36]), and therefore $K$ is finite.

Next, we will need the fact that hyperbolic groups have finitely many cone types. Recall the following definition.

Definition 1.19. Let $\Gamma$ be a graph, let $G$ be a group acting by isometries on $\Gamma$, and fix a vertex $x_{0} \in \Gamma$. For each $x \in \Gamma$, the cone on $x$ is the set

$$
C(x)=\left\{y \in \Gamma \mid d\left(x_{0}, y\right)=d\left(x_{0}, x\right)+d(x, y)\right\} .
$$

Two points $x, x^{\prime} \in \Gamma$ have the same cone type if there exists a $g \in G$ so that $g x=x^{\prime}$ and $g C(x)=C\left(x^{\prime}\right)$.

Note that a point $y$ lies in a cone $C(x)$ if and only if there exists a geodesic from $x_{0}$ to $y$ that goes through $x$. Figure 3 shows the cone types for the 1-skeleton of the order five square tiling of the hyperbolic plane, where $G$ is the full isometry group.

The following theorem is due to Cannon [12] (see [10, Theorem 2.18]).

Theorem 1.20 (Cannon). Let $\Gamma$ be a hyperbolic graph, let $x_{0} \in \Gamma$, and let $G$ be a group acting properly and cocompactly by isometries on $\Gamma$. Then $\Gamma$ has only finitely many cone types with respect to $G$ and $x_{0}$.

1.3. The horofunction boundary. Here we review the basic definition and properties of the horofunction boundary (or metric boundary) of a locally finite, connected graph. The horofunction boundary can actually be defined for any complete metric space; see [10] for a general introduction. 


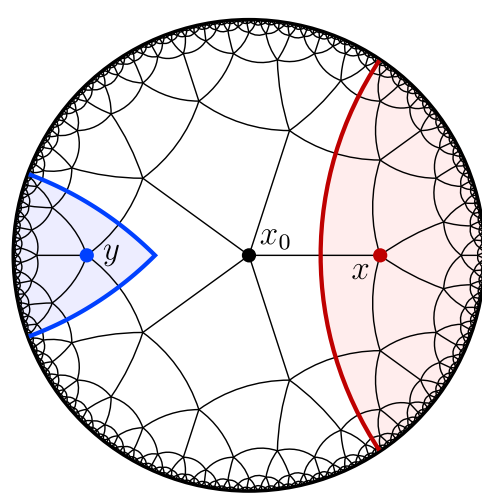

(a)

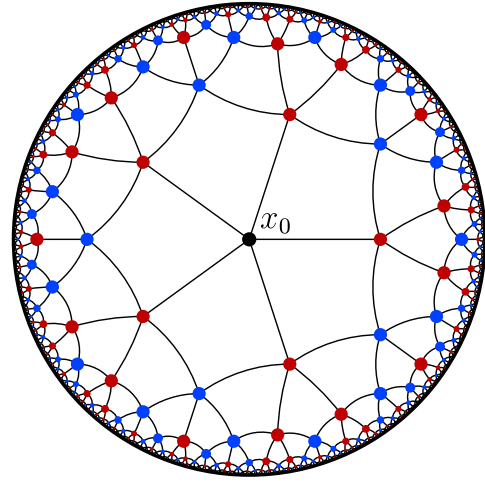

(b)

Figure 3. (a) Cones $C(x)$ and $C(y)$ for two points $x$ and $y$ in a hyperbolic graph $\Gamma$, where the cone consists of all vertices in the shaded region. (b) Cone types in $\Gamma$ if $G$ is the full isometry group. Red vertices have the same cone type as $x$ and blue vertices have the same cone type as $y$.

Let $\Gamma$ be a locally finite, connected graph. As before, we put the path metric on $\Gamma$, and we identify $\Gamma$ with is vertex set. Let $F(\Gamma, \mathbb{Z})$ be the abelian group of all integer-valued functions on $\Gamma$, and let $\bar{F}(\Gamma, \mathbb{Z})$ be the quotient of $F(\Gamma, \mathbb{Z})$ by the subgroup of constant functions. That is, two functions $f, g \in F(\Gamma, \mathbb{Z})$ are identified in $\bar{F}(\Gamma, \mathbb{Z})$ if $f-g$ is a constant function.

Notation 1.21. If $f \in F(\Gamma, \mathbb{Z})$, we will let $\bar{f}$ denote the corresponding element of $\bar{F}(\Gamma, \mathbb{Z})$.

Note that $F(\Gamma, \mathbb{Z})=\mathbb{Z}^{\Gamma}$ forms a topological space under the product topology, from which $\bar{F}(\Gamma, \mathbb{Z})$ inherits a quotient topology.

\section{Definition 1.22.}

(1) If $x \in \Gamma$, the associated distance function is the function $d_{x}: \Gamma \rightarrow \mathbb{Z}$ defined by

$$
d_{x}(y)=d(x, y)
$$

for all $y \in \Gamma$.

(2) The canonical embedding $i: \Gamma \rightarrow \bar{F}(\Gamma, \mathbb{Z})$ is the map defined by

$$
i(x)=\bar{d}_{x}
$$

for all $x \in \Gamma$.

Note that each $\bar{d}_{x}$ is an isolated point in $i(\Gamma)$, since $\bar{d}_{x}$ has a global minimum at $x$. Thus $i$ really is an embedding. 


\section{Definition 1.23.}

(1) The horofunction boundary of $\Gamma$, denoted $\partial_{h} \Gamma$, is the set of limit points of $i(\Gamma)$ in $\bar{F}(\Gamma, \mathbb{Z})$.

(2) A function $f: \Gamma \rightarrow \mathbb{Z}$ is called a horofunction if $\bar{f} \in \partial_{h} \Gamma$.

Note that if $f$ is a horofunction then so is $f+C$ for any constant $C \in \mathbb{Z}$, and these correspond to the same point in the horofunction boundary.

Note also that $\partial_{h} \Gamma$ is empty when $\Gamma$ is finite, since $i(\Gamma)$ cannot have any limit points. Thus a finite graph has no horofunctions.

Example 1.24. If $\Gamma$ is an infinite path with vertex set $\mathbb{N}$, then $\partial_{h} \Gamma$ is a single point. In particular, the only horofunctions on $\Gamma$ are

$$
f(n)=-n+C
$$

for $C \in \mathbb{Z}$ a constant. Note that $\bar{d}_{m} \rightarrow \bar{f}$ in $\bar{F}(\Gamma, \mathbb{Z})$ as $m \rightarrow \infty$. In particular,

$$
d_{m}(n)=|m-n|= \begin{cases}-n+m & \text { if } n \leq m, \\ n-m & \text { if } n>m,\end{cases}
$$

for all $m \in \mathbb{N}$, so $\bar{d}_{m}$ agrees with $\bar{f}$ on $\mathbb{N} \cap[0, m]$.

Example 1.25. If $\Gamma$ is a bi-infinite path with vertex set $\mathbb{Z}$, then $\partial_{h} \Gamma$ has two points, corresponding to the horofunctions

$$
f_{-\infty}(n)=n+C \quad \text { and } \quad f_{\infty}(n)=-n+C .
$$

Again $\bar{d}_{m} \rightarrow \bar{f}_{\infty}$ as $m \rightarrow \infty$, and $\bar{d}_{m} \rightarrow \bar{f}_{-\infty}$ as $m \rightarrow-\infty$.

Example 1.26. Let $\Gamma$ be the infinite square grid in the plane, with vertex set $\mathbb{Z}^{2}$. Then $\partial_{h} \Gamma$ is homeomorphic to the union

$$
(\mathbb{Z} \times\{ \pm \infty\}) \cup(\{ \pm \infty\} \times \mathbb{Z}) \cup(\{ \pm \infty\} \times\{ \pm \infty\})
$$

with the obvious topology [18]. For example, the horofunction

$$
f(x, y)=-x-y+C
$$

corresponds to the point $(\infty, \infty)$, and the horofunction

$$
f(x, y)=|x-5|-y+C
$$

corresponds to the point $(5, \infty)$.

Proposition 1.27. Let $f: \Gamma \rightarrow \mathbb{Z}$. Then the following are equivalent:

(1) $f$ is a horofunction on $\Gamma$.

(2) For every finite set $B \subseteq \Gamma$ there exist infinitely many $x \in \Gamma \backslash B$ for which $\bar{d}_{x}$ agrees with $\bar{f}$ on $B$. 
Proof. For any finite set $B \subseteq \Gamma$, let

$$
U_{B}=\{\bar{g} \in \bar{F}(\Gamma, \mathbb{Z}) \mid \bar{g} \text { agrees with } \bar{f} \text { on } B\} .
$$

It is easy to check that the sets $U_{B}$ form a neighborhood base for $\bar{F}(\Gamma, \mathbb{Z})$ at $\bar{f}$, so $f$ satisfies condition (2) if and only if $\bar{f}$ is a limit point of $i(\Gamma)$.

Proposition 1.28. If $\Gamma$ is a locally finite, connected graph, then $\partial_{h} \Gamma$ is compact and totally disconnected.

Proof. Fix a point $x_{0} \in \Gamma$. For each $x \in \Gamma$, let $d_{x}^{\prime}: \Gamma \rightarrow \mathbb{Z}$ be the function

$$
d_{x}^{\prime}(y)=d_{x}(y)-d_{x}\left(x_{0}\right) .
$$

Note then that $d_{x}^{\prime}\left(x_{0}\right)=0$ and $\bar{d}_{x}^{\prime}=\bar{d}_{x}$. By the triangle inequality, we know that

$$
\left|d_{x}^{\prime}(y)\right| \leq d\left(x_{0}, y\right)
$$

for all $y \in \Gamma$, so each $d_{x}^{\prime}$ lies in the infinite product

$$
S=\prod_{y \in \Gamma}\left(\mathbb{Z} \cap\left[-d\left(x_{0}, y\right), d\left(x_{0}, y\right)\right]\right) .
$$

This is a product of finite sets, which means that $S$ is totally disconnected and compact. Since $f\left(x_{0}\right)=0$ for all $f \in S$, the quotient $\operatorname{map} F(\Gamma, \mathbb{Z}) \rightarrow \bar{F}(\Gamma, \mathbb{Z})$ is one-to-one on $S$, so the image $\bar{S}$ of $S$ in $\bar{F}(\Gamma, \mathbb{Z})$ is homeomorphic to $S$. But $i(\Gamma) \subseteq \mathcal{S}$, so $\partial_{h} \Gamma \subseteq \bar{S}$ since $\bar{S}$ is closed, and the result follows.

Now suppose that $G$ is a group acting by isometries on $\Gamma$. There is natural left action of $G$ on $F(\Gamma, \mathbb{Z})$ defined by

$$
(g f)(p)=f\left(g^{-1} p\right)
$$

for all $g \in G, f \in F(\Gamma, \mathbb{Z})$, and $p \in \Gamma$, and this descends to a left action of $G$ on $\bar{F}(\Gamma, \mathbb{Z})$. It is easy to check that the canonical embedding $i: \Gamma \rightarrow \bar{F}(\Gamma, \mathbb{Z})$ is equivariant with respect to this action. In particular, $g f$ is a horofunction on $\Gamma$ for any $g \in G$ and any horofunction $f \in F(\Gamma, \mathbb{Z})$, and this gives us a left action of $G$ on $\partial_{h} \Gamma$.

Theorem 1.29. Let $\Gamma$ be a $\delta$-hyperbolic graph with Gromov boundary $\partial \Gamma$ and horofunction boundary $\partial_{h} \Gamma$, and let $G$ be a group acting by isometries on $\Gamma$. Then there exists a $G$-equivariant quotient map $q: \partial_{h} \Gamma \rightarrow \partial \Gamma$.

Proof. See [48]. Note that $\partial \Gamma$ and $\partial_{h} \Gamma$ are both compact Hausdorff spaces in this case, so the surjection $\partial_{h} \Gamma \rightarrow \partial \Gamma$ defined in [48] is indeed a quotient map.

Thus, if we wish to show that $G$ acts rationally on $\partial \Gamma$, it suffices to show that $G$ acts rationally on $\partial_{h} \Gamma$. That is, if we wish to prove Theorem 3 (and hence Theorem 1), it suffices to prove the following: 
Theorem 1.30. Let $\Gamma$ be a $\delta$-hyperbolic graph with horofunction boundary $\partial_{h} \Gamma$, and let $G$ be a group acting properly and cocompactly by isometries on $\Gamma$. Then the induced action of $G$ on $\partial_{h} \Gamma$ is rational.

Sections 2 and 3 are devoted to a proof of this theorem.

Remark 1.31. The horofunction boundary defined here is sometimes called the "metric boundary", and was introduced by Gromov in the study of nonpositively curved manifolds [3]. The definition of horofunction that we are using is one of two definitions suggested by Gromov in his 1987 essay introducing hyperbolic groups [26], the other of which involves quasiconvex functions satisfying a distancelike condition, and was later refined by Coornaert and Papadopolous. These two definitions are inequivalent, e.g. in the case of $\mathbb{Z} \times \mathbb{Z}_{3}$ [1], though as M. Kapovich observes these are equivalent up to uniformly bounded error, which is sufficient for most geometric applications.

Coornaert and Papadopoulos have used their version of Gromov's quasiconvex horofunction definition to provide a precise statement and proof of Gromov's assertion that the space $\Phi_{0}$ of integral cocycles is a subshift of finite type [15,16], as well as to provide a symbolic coding for the associated geodesic flow [14]. Our binary coding of the horofunction boundary $\partial_{h} \Gamma$ is similar in spirit to their coding of the space $\Phi_{0}$ of integral quasiconvex cocycles by a subshift of finite type, but there does not seem to be any direct translation between them, nor have we found a way to use their coding to show that the natural action of $G$ on $\Phi_{0}$ is rational.

\section{Rational groups for self-similar trees}

As we have seen, there is one version $\mathbb{R}_{A}$ of the rational group $\mathbb{R}$ for each finite set $A$. In this section, we generalize the definition of $R_{A}$ to allow for rational homeomorphisms between the boundaries of arbitrary self-similar trees. We will construct such a tree in Section 3 for an arbitrary hyperbolic group.

As defined below, self-similar trees do not necessarily have canonical isomorphisms between subtrees, and therefore infinite descending paths in self-similar trees do not correspond to infinite strings of symbols in a natural way. As a result, the theory of rational homeomorphisms defined by transducers does not directly apply to self-similar trees. In this section, we develop the theory of rational homeomorphisms of the boundaries of self-similar trees using a generalization of the notion of having finitely many restrictions, and then prove that the corresponding rational groups embed in $R$.

Readers interested primarily in hyperbolic groups may want to skip over some of the technical development in this section. For such readers, we recommend reading the definition of a self-similar tree (Definition 2.1), the definition of a rational homeomorphism (Definition 2.8), and our most general result on rational actions (Corollary 2.31) before continuing to Section 3. 
We will use the following notation and terminology for trees throughout this section:

- We will identify each tree $T$ with its vertex set. In particular, the notation $v \in T$ will mean that $v$ is a vertex of $T$.

- The depth of a vertex $v \in T$, denoted $|v|$, is the distance from $v$ to the root of $T$.

- If $v \in T$, we let $T_{v}$ denote the subtree of $T$ consisting of $v$ and all of its descendants.

- If $T$ is a locally finite rooted tree, the boundary of $T$ is the space $\partial T$ of all infinite descending paths in $T$ starting with the root.

- If $v \in T$, we will think of $\partial T_{v}$ as a subset of $\partial T$, namely the set of all infinite descending paths that go through $v$. Such subsets are clopen in $\partial T$, and form a basis for the topology on $\partial T$.

- If $S \subseteq \partial T$ has at least two points, the deepest parent of $S$ is the deepest vertex $v$ for which $S \subseteq T_{v}$.

- The standard ultrametric on $\partial T$ is the metric $d: \partial T \times \partial T \rightarrow \mathbb{R}$ defined by

$$
d(p, q)=2^{-|v|}
$$

for $p \neq q$, where $v$ is the deepest parent of $\{p, q\}$.

- If $\varphi: T_{v} \rightarrow T_{w}$ is a rooted isomorphism between subtrees of $T$, we will let $\varphi_{*}$ denote the induced homeomorphism $\partial T_{v} \rightarrow \partial T_{w}$. Note that $\varphi_{*}$ is a similarity transformation with respect to the standard ultrametric, with

$$
d\left(\varphi_{*}(p), \varphi_{*}(q)\right)=2^{|v|-|w|} d(p, q)
$$

for all $p, q \in \partial T_{v}$.

\subsection{Self-similar trees.}

Definition 2.1. Let $T$ be a locally finite rooted tree. A self-similar structure on $T$ consists of the following data:

(1) A partition of the vertices of $T$ into finitely many types.

(2) For every pair $u, v$ of vertices of $T$ of the same type, a nonempty, finite set $\operatorname{Mor}(u, v)$ of (rooted) tree isomorphisms $T_{u} \rightarrow T_{v}$, each of which maps vertices of $T_{u}$ to vertices of $T_{v}$ of the same type.

Elements of $\operatorname{Mor}(u, v)$ are called morphisms, and are required to satisfy the following conditions:

(a) If $\varphi \in \operatorname{Mor}(u, v)$, then $\varphi^{-1} \in \operatorname{Mor}(v, u)$.

(b) If $\varphi \in \operatorname{Mor}(u, v)$ and $\psi \in \operatorname{Mor}(v, w)$, then $\psi \varphi \in \operatorname{Mor}(u, w)$.

(c) If $\varphi \in \operatorname{Mor}(v, w)$ and $u \in T_{v}$, then the restriction $\left.\varphi\right|_{T_{u}}: T_{u} \rightarrow T_{\varphi(u)}$ is in $\operatorname{Mor}(u, \varphi(u))$.

A self-similar tree is a locally finite rooted tree $T$ together with a self-similar structure on $T$. 
Note 2.2. We will have some use for partial compositions of morphisms. If $\varphi \in$ $\operatorname{Mor}(v, w), \psi \in \operatorname{Mor}(x, y)$, and $T_{w} \subseteq T_{x}$, we will let $\psi \varphi$ denote the composition

$$
T_{v} \stackrel{\varphi}{\rightarrow} T_{w} \stackrel{\psi^{\prime}}{\rightarrow} T_{\psi(w)},
$$

where $\psi^{\prime}$ is the restriction of $\psi$. Similarly, if $\varphi \in \operatorname{Mor}(v, w), \psi \in \operatorname{Mor}(x, y)$, and $T_{x} \subseteq T_{w}$, we will let $\psi \varphi$ denote the composition

$$
T_{\varphi^{-1}(x)} \stackrel{\varphi^{\prime}}{\rightarrow} T_{x} \stackrel{\psi}{\rightarrow} T_{y},
$$

where $\varphi^{\prime}$ is the restriction of $\varphi$.

Using this notion of composition, conditions (a) through (c) can be summarized by saying that the collection of all morphisms forms an inverse semigroup of isomorphisms between subtrees of $T$.

Example 2.3. If $A$ is a finite alphabet, then we can give $A^{*}$ the structure of a self-similar tree as follows:

(1) There is only one type of vertex in $A^{*}$.

(2) For each $\alpha, \beta \in A^{*}$, there is only one morphism $\varphi_{\alpha \beta}: A_{\alpha}^{*} \rightarrow A_{\beta}^{*}$, namely the prefix replacement

$$
\varphi_{\alpha \beta}(\alpha \gamma)=\beta \gamma \quad\left(\gamma \in A^{\omega}\right) .
$$

It is easy to check that this satisfies the required axioms.

In general, we say that a self-similar structure on a tree $T$ is rigid if there is at most one morphism between any two vertices of $T$. A self-similar tree whose self-similar structure is rigid is said to be a rigid tree.

The previous example put a rigid self-similar structure on $A^{*}$, but other selfsimilar structures are possible.

Example 2.4. Let $A$ be a finite alphabet, and let $G$ be a group of automorphisms of $A^{*}$. Following Nekrashevych [38], we say that $G$ is self-similar if it is closed under restrictions, i.e. for each $g \in G$ and $\alpha \in A^{*}$ the automorphism $\left.g\right|_{\alpha}: A^{*} \rightarrow A^{*}$ defined by

$$
g(\alpha \gamma)=\left.g(\alpha) g\right|_{\alpha}(\gamma) \quad\left(\gamma \in A^{*}\right)
$$

is again an element of $G$.

Now suppose that $G$ is a finite self-similar subgroup of $\operatorname{Aut}\left(A^{*}\right)$. For example, if $A$ has $n$ elements, then the symmetric group $S_{n}$ acts on $A^{*}$ by permuting symbols, and the image of $S_{n}$ in $\operatorname{Aut}\left(A^{*}\right)$ is a finite self-similar group. Then $G$ induces a self-similar structure on $A^{*}$ as follows:

(1) There is only one type of vertex in $A^{*}$.

(2) For each $g \in G$ and $\alpha, \beta \in A^{*}$, there is a morphism $g_{\alpha \beta}$ : $A_{\alpha}^{*} \rightarrow A_{\beta}^{*}$ defined by

$$
g_{\alpha \beta}(\alpha \gamma)=\beta g(\gamma) \quad\left(\gamma \in A^{*}\right) .
$$

It is easy to check that this satisfies the required axioms. 

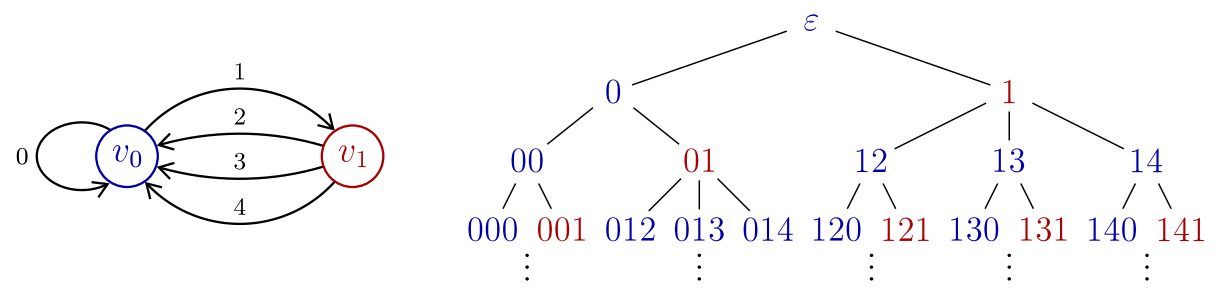

Figure 4. A directed multigraph $\Gamma$ and the corresponding path language tree $\mathcal{L}\left(\Gamma, v_{0}\right)$. Vertices of type $v_{0}$ are shown in blue, and vertices of type $v_{1}$ are shown in red.

Of course, there are also self-similar trees with more than one type of vertex.

Example 2.5. Let $\Gamma=(V, E)$ be a finite directed multigraph with edge set $E$, and fix an initial vertex $v_{0} \in V$. The corresponding path language $\mathcal{L}\left(\Gamma, v_{0}\right) \subseteq E^{*}$ is the set of all finite directed paths $e_{1} e_{2} \cdots e_{n}$ in $\Gamma$ that begin at $v_{0}$. This set has the natural structure of a locally finite tree, whose root is the empty path $\varepsilon$, and whose boundary $\partial \mathcal{L}\left(\Gamma, v_{0}\right)$ is the set of all infinite directed paths in $\Gamma$ starting at $v_{0}$. An example of such a tree is shown in Figure 4.

We can define a self-similar structure on $\mathcal{L}\left(\Gamma, v_{0}\right)$ as follows:

(1) Two paths $p, q \in \mathcal{L}\left(\Gamma, v_{0}\right)$ have the same type if and only if $p$ and $q$ end at the same vertex of $\Gamma$.

(2) For each pair $p, q$ of paths ending at the same vertex $w$, we define a single morphism $\varphi_{p q}: \mathcal{L}\left(\Gamma, v_{0}\right)_{p} \rightarrow \mathcal{L}\left(\Gamma, v_{0}\right)_{q}$ by

$$
\varphi_{p q}(p r)=q r
$$

for every finite directed path $r$ in $\Gamma$ starting at $w$. We will refer to $\varphi_{p q}$ as a prefix replacement morphism.

It is easy to check that this satisfies the required axioms, and gives $\mathcal{L}\left(\Gamma, v_{0}\right)$ the structure of a rigid tree.

It turns out that, for any self-similar tree $T$, the underlying tree is isomorphic to a path language as described above. In particular, define the type graph $\Gamma$ of a self-similar tree $T$ as follows:

(1) There is one vertex in $\Gamma$ for each vertex type in $T$.

(2) Given a pair $t_{1}, t_{2}$ of vertices in $\Gamma$, the number of directed edges in $\Gamma$ from $t_{1}$ to $t_{2}$ is equal to the number of children of type $t_{2}$ that each vertex of type $t_{1}$ has in $T$.

Then it is easy to construct an isomorphism of trees $\mathcal{L}\left(\Gamma, t_{0}\right) \rightarrow T$, where $t_{0}$ is the type of the root vertex of $T$, though the morphisms of $\mathcal{L}\left(\Gamma, t_{0}\right)$ need not be the same as the morphisms of $T$. However, we will show in Proposition 2.21 that, in the case 
of a rigid tree, it is always possible to construct an isomorphism from a path language that respects the self-similar structure.

2.2. Rational homeomorphisms. Our next goal is to define the group of rational homeomorphisms of $\partial T$.

Definition 2.6. Let $T$ and $T^{\prime}$ be self-similar trees, let $f: \partial T \rightarrow \partial T^{\prime}$ be a homeomorphism, and let $v$ and $w$ be vertices of $T$ of the same type. We say that $f$ has equivalent restrictions at $v$ and $w$ if there exist vertices $x$ and $y$ of $T^{\prime}$ of the same type and morphisms $\varphi: T_{v} \rightarrow T_{w}$ and $\psi: T_{x}^{\prime} \rightarrow T_{y}^{\prime}$ such that $f\left(\partial T_{v}\right) \subseteq \partial T_{x}^{\prime}$, $f\left(\partial T_{w}\right) \subseteq \partial T_{y}^{\prime}$, and the diagram

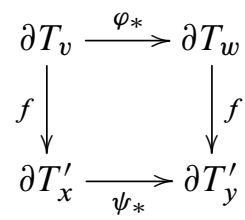

commutes, where $\varphi_{*}: \partial T_{v} \rightarrow \partial T_{w}$ and $\psi_{*}: \partial T_{x}^{\prime} \rightarrow \partial T_{y}^{\prime}$ are the induced homeomorphisms.

Proposition 2.7. Let $f: \partial T \rightarrow \partial T^{\prime}$ be a function. Then " $f$ has equivalent restrictions at $v$ and $w$ ” is an equivalence relation on vertices of $T$.

Proof. For the reflexive property, if $v$ is a vertex of $T$, then it suffices to let $\varphi=\mathrm{id}_{v}$, $x$ and $y$ be the root of $T^{\prime}$, and $\psi=\mathrm{id}_{x}=\mathrm{id}_{y}$. The symmetric property is clear, by inversion of $\varphi$ and $\psi$.

For the transitive property, let $u, v$, and $w$ be vertices of $T$ of the same type, and suppose $f$ has equivalent restrictions at $u$ and $v$ and also at $v$ and $w$. Then there exist vertices $x, y$ of $T^{\prime}$ of the same type and $y^{\prime}, z$ of $T^{\prime}$ of the same type with

$$
f\left(\partial T_{u}\right) \subseteq \partial T_{x}^{\prime}, \quad f\left(\partial T_{v}\right) \subseteq \partial T_{y}^{\prime} \cap \partial T_{y^{\prime}}^{\prime}, \quad f\left(\partial T_{w}\right) \subseteq \partial T_{z}^{\prime}
$$

and morphisms $\varphi \in \operatorname{Mor}(u, v), \varphi^{\prime} \in \operatorname{Mor}(v, w), \psi \in \operatorname{Mor}(x, y)$, and $\psi^{\prime} \in$ $\operatorname{Mor}\left(y^{\prime}, z\right)$ making the following diagrams commute:

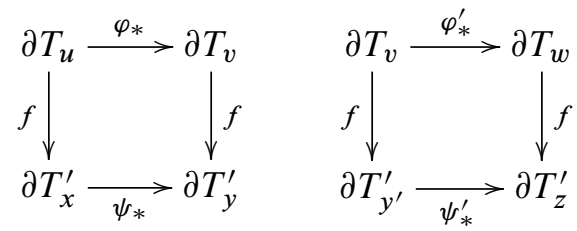

Note that either $y=y^{\prime}$, or $y$ is a descendant of $y^{\prime}$, or $y^{\prime}$ is a descendant of $y$. If $y=y^{\prime}$ then we are done, since it suffices to use $x, z$, and the compositions $\varphi^{\prime} \circ \varphi \in \operatorname{Mor}(u, w)$ and $\psi^{\prime} \circ \psi \in \operatorname{Mor}(x, z)$. If $y$ is a descendant of $y^{\prime}$, then 
we can replace $y^{\prime}$ by $y, z$ by $\psi^{\prime}(y)$, and $\psi^{\prime}$ by its restriction to arrive in the case where $y=y^{\prime}$. Similarly, if $y^{\prime}$ is a descendant of $y$, then we can replace $y$ by $y^{\prime}, x$ by $\psi^{-1}\left(y^{\prime}\right)$, and $\psi$ by its restriction to arrive in the case where $y=y^{\prime}$.

Definition 2.8. Let $T$ and $T^{\prime}$ be self-similar trees. A homeomorphism $f: \partial T \rightarrow \partial T^{\prime}$ is rational if it has only finitely many different equivalence classes of restrictions.

We will show in Proposition 2.10 that this definition of a rational homeomorphism is a generalization of the definition given in [24]. First we need the following fundamental proposition.

Proposition 2.9. Let $T$ and $T^{\prime}$ be self-similar trees, let $f: \partial T \rightarrow \partial T^{\prime}$ be a homeomorphism, and let $v$ and $w$ be vertices of $T$ of the same type. Suppose that $f\left(\partial T_{v}\right)$ and $f\left(\partial T_{w}\right)$ each have at least two points, let $x$ be the deepest parent of $f\left(\partial T_{v}\right)$ in $T^{\prime}$, and let $y$ be the deepest parent of $f\left(\partial T_{w}\right)$ in $T^{\prime}$. Then $f$ has equivalent restrictions at $v$ and $w$ if and only if $x$ and $y$ have the same type and there exist morphisms $\varphi: T_{v} \rightarrow T_{w}$ and $\psi: T_{x}^{\prime} \rightarrow T_{y}^{\prime}$ making the following diagram commute:

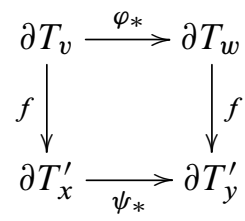

Proof. Clearly the given condition implies that $f$ has equivalent restrictions at $v$ and $w$. For the converse, suppose that $f$ has equivalent restrictions at $v$ and $w$. Then there exist vertices $r$ and $s$ of $T^{\prime}$ and morphisms $\varphi: T_{v} \rightarrow T_{w}$ and $\chi: T_{r}^{\prime} \rightarrow T_{s}^{\prime}$ such that $f\left(\partial T_{v}\right) \subseteq \partial T_{r}^{\prime}, f\left(\partial T_{w}\right) \subseteq \partial T_{s}^{\prime}$, and the diagram

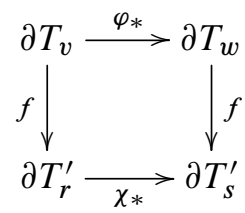

commutes. Since $f\left(\partial T_{v}\right) \subseteq \partial T_{r}^{\prime}$ and $x$ is the deepest parent of $f\left(\partial T_{v}\right)$, we know that $x$ is a descendant of $r$, and similarly $y$ is a descendant of $s$. We claim that $\chi(x)=y$.

Since $\varphi_{*}$ is a homeomorphism, we know that $\varphi_{*}\left(\partial T_{v}\right)=\partial T_{w}$, so

$$
f\left(\partial T_{w}\right)=f\left(\varphi_{*}\left(\partial T_{v}\right)\right)=\chi_{*}\left(f\left(\partial T_{v}\right)\right) \subseteq \chi_{*}\left(\partial T_{x}^{\prime}\right)=\partial T_{\chi(x)}^{\prime} .
$$

Since $y$ is the deepest parent of $f\left(\partial T_{w}\right)$, it follows that $y$ is a descendant of $\chi(x)$. But similarly,

$$
f\left(\partial T_{v}\right)=f\left(\varphi_{*}^{-1}\left(\partial T_{w}\right)\right)=\chi_{*}^{-1}\left(f\left(\partial T_{w}\right)\right) \subseteq \chi_{*}^{-1}\left(\partial T_{y}\right)=\partial T_{\chi^{-1}(y)}
$$


so $x$ must be a descendant of $\chi^{-1}(y)$, and therefore $\chi(x)=y$. Since $\chi$ is a morphism, it follows that $x$ and $y$ have the same type, and the restriction $\psi: T_{x}^{\prime} \rightarrow T_{y}^{\prime}$ of $\chi$ is a morphism with the desired properties.

For the following proposition, recall from Example 2.3 that if $A$ is a finite alphabet then $A^{*}$ has the natural structure of a self-similar tree. The boundary $\partial A^{*}$ of this tree is homeomorphic to $A^{\omega}$.

Proposition 2.10. Let $A_{1}$ and $A_{2}$ be finite alphabets with at least two symbols. Then a homeomorphism $f: A_{1}^{\omega} \rightarrow A_{2}^{\omega}$ is rational in the sense of Definition 2.8 if and only if it is rational in the sense of Definition 1.2.

Proof. Let $\alpha, \beta \in A_{1}^{*}$. Let $\gamma, \delta \in A_{2}^{*}$ be the greatest common prefixes (i.e. deepest parents) of $f\left(\partial\left(A_{1}^{*}\right)_{\alpha}\right)$ and $f\left(\partial\left(A_{1}^{*}\right)_{\beta}\right)$, respectively. Then the restrictions $\left.f\right|_{\alpha}$ and $\left.f\right|_{\beta}$ defined in Definition 1.7 are equal if and only if the diagram

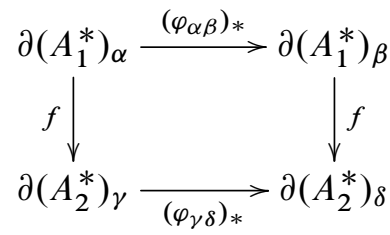

commutes, where $\varphi_{\alpha \beta}:\left(A_{1}^{*}\right)_{\alpha} \rightarrow\left(A_{1}^{*}\right)_{\beta}$ and $\varphi_{\gamma \delta}:\left(A_{2}^{*}\right)_{\gamma} \rightarrow\left(A_{2}^{*}\right)_{\delta}$ are the prefix replacement morphisms. By Proposition 2.9, the diagram above commutes if and only if $f$ has equivalent restrictions at $\alpha$ and $\beta$ in the sense of Definition 2.6, and therefore $\left.f\right|_{\alpha}=\left.f\right|_{\beta}$ if and only if $f$ has equivalent restrictions at $\alpha$ and $\beta$ in the sense of Definition 2.6. The result now follows immediately from Definition 2.8 and Theorem 1.8 .

Remark 2.11. In an effort to simplify the exposition, we are only considering rational homeomorphisms between the boundaries of self-similar trees. It would be possible to develop a more general theory of rational functions by including a requirement similar to condition (2) in Theorem 1.8. In particular, if $T^{\prime}$ is a self-similar tree, we say that a point $p \in \partial T^{\prime}$ is a rational point if there exist distinct $x, y \in T^{\prime}$ with $p \in \partial T_{y}^{\prime} \subseteq \partial T_{x}^{\prime}$ and a morphism $\varphi: T_{x}^{\prime} \rightarrow T_{y}^{\prime}$ such that $\varphi_{*}(p)=p$. These are the analogs in $\partial T^{\prime}$ of eventually periodic points in $A^{*}$ for a finite alphabet $A$. We could then define a continuous function $f: \partial T \rightarrow \partial T^{\prime}$ to be rational if it satisfies the following conditions:

(1) $f$ has finitely many equivalence classes of restrictions.

(2) For each $v \in T$, if $f\left(\partial T_{v}\right)$ is a single point, then this must be a rational point in $\partial T^{\prime}$.

This would agree with the existing definition of rational in the case of a continuous function $A_{1}^{\omega} \rightarrow A_{2}^{\omega}$, where $A_{1}$ and $A_{2}$ are finite alphabets. 
We now wish to prove that the rational homeomorphisms of $\partial T$ form a group. To simplify the initial development of our theory, we will restrict our class of self-similar trees.

Definition 2.12. A self-similar tree $T$ is branching if every vertex in $T$ has at least two children.

If $T$ is branching, then $\partial T$ has no isolated points, and is therefore homeomorphic to the Cantor set. Each basic clopen set $\partial T_{v}$ is also homeomorphic to a Cantor set, and has $v$ as its deepest parent. Moreover, if $T$ and $T^{\prime}$ are branching and $f: \partial T \rightarrow \partial T^{\prime}$ is a rational homeomorphism, then $\partial T_{v}$ has at least two points for every $v \in V$, so $f\left(\partial T_{v}\right)$ always has a deepest parent.

For now, we will develop our theory only for branching self-similar trees, though we will extend to a larger class of self-similar trees in Section 2.5.

Proposition 2.13. Let $T, T^{\prime}, T^{\prime \prime}$ be branching self-similar trees, and let $f: \partial T \rightarrow \partial T^{\prime}$ and $g: \partial T^{\prime} \rightarrow \partial T^{\prime \prime}$ be rational homeomorphisms. Then the composition

$$
g \circ f: \partial T \rightarrow \partial T^{\prime \prime}
$$

is rational.

Proof. Let $E$ be an equivalence class of vertices of $T$ under the equivalence relation " $f$ has equivalent restrictions at $v$ and $w$ ". Note that there are only finitely many such $E$, since $f$ is rational. Therefore, it suffices to prove that $g \circ f$ has finitely many equivalence classes of restrictions on $E$.

For each $v \in E$, let $r(v)$ denote the deepest parent of $f\left(\partial T_{v}\right)$ in $T^{\prime}$, which exists since $T$ and $T^{\prime}$ are branching. By Proposition 2.9, for every pair of vertices $v, w \in E$, there exist a pair of morphisms $\varphi_{v w}: T_{v} \rightarrow T_{w}$ and $\psi_{v w}: T_{r(v)}^{\prime} \rightarrow T_{r(w)}^{\prime}$ making the following diagram commute:

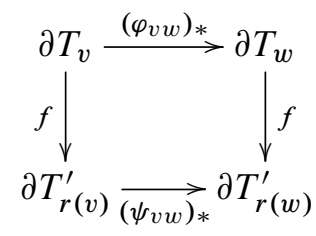

Put an equivalence on $E$ by $v \sim w$ if $g$ has equivalent restrictions at $r(v)$ and $r(w)$. Since $g$ is rational, there are only finitely many equivalence classes. Let $E^{\prime}$ be such an equivalence class. It suffices to show that $g \circ f$ has finitely many equivalence classes of restrictions on $E^{\prime}$.

For each $v \in E^{\prime}$, let $s(v)$ denote the deepest parent of $g\left(\partial T_{r(v)}^{\prime}\right)$ in $T^{\prime \prime}$, which exists since $T^{\prime}$ and $T^{\prime \prime}$ are branching. By Proposition 2.9, for each $v, w \in E^{\prime}$, there 
exist morphisms $\mu_{v w}: T_{r(v)}^{\prime} \rightarrow T_{r(w)}^{\prime}$ and $v_{v w}: T_{s(v)}^{\prime \prime} \rightarrow T_{s(w)}^{\prime \prime}$ making the following diagram commute:

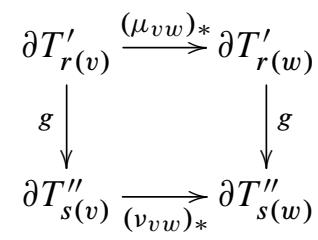

Fix a vertex $u \in E^{\prime}$. For each $v \in E^{\prime}$ let $\pi_{v}: T_{r(u)} \rightarrow T_{r(u)}$ be the morphism $\pi_{v}=\psi_{u v}^{-1} \mu_{u v}$. We claim that, for $v, w \in E^{\prime}$, the composition $g \circ f$ has equivalent restrictions at $v$ and $w$ whenever $\pi_{v}=\pi_{w}$. Since $\operatorname{Mor}(r(u), r(u))$ is finite, it will follow immediately from this that $g \circ f$ has only finitely many different restrictions on $E^{\prime}$.

Let $v, w \in E^{\prime}$ and suppose that $\pi_{v}=\pi_{w}$. Then $\psi_{u v}^{-1} \mu_{u v}=\psi_{u w}^{-1} \mu_{u w}$, so the following diagram commutes, including the central square if we allow inverse arrows:

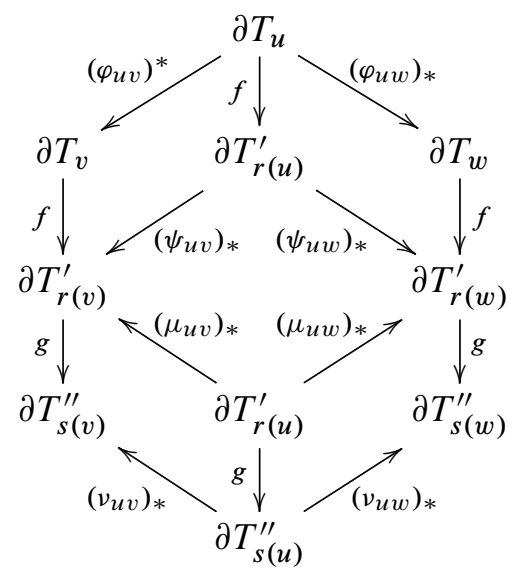

In particular, the outer octagon gives us the commutative square

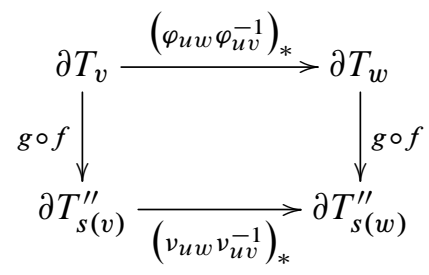

and therefore $g \circ f$ has equivalent restrictions at $v$ and $w$.

Proposition 2.14. Let $T$ and $T^{\prime}$ be branching self-similar trees, and let $f: \partial T \rightarrow \partial T^{\prime}$ be a rational homeomorphism. Then the inverse $f^{-1}: \partial T^{\prime} \rightarrow \partial T$ is rational. 
Proof. For each $v$ in $T^{\prime}$, let $r(v)$ be the deepest parent of $f^{-1}\left(T_{v}^{\prime}\right)$ in $T$, which exists since $T$ and $T^{\prime}$ are branching. Put an equivalence relation $\sim$ on the vertices of $T^{\prime}$ by $v \sim w$ if $f$ has equivalent restrictions at $\partial T_{r(v)}$ and $\partial T_{r(w)}$. Since $f$ is rational, there are only finitely many such equivalence classes. Let $E$ be such an equivalence class. It suffices to prove that $f^{-1}$ has only finitely many different restrictions on $E$.

Fix a vertex $u \in E$, and for each $v \in E$ let $s(v)$ be the deepest parent of $f\left(\partial T_{r(v)}\right)$ in $T^{\prime}$, which exists since $T$ and $T^{\prime}$ are branching. By Proposition 2.9, for each $v \in E$ there exist morphisms $\varphi_{v}: T_{r(v)} \rightarrow T_{r(u)}$ and $\psi_{v}: T_{s(v)}^{\prime} \rightarrow T_{s(u)}^{\prime}$ making the following diagram commute:

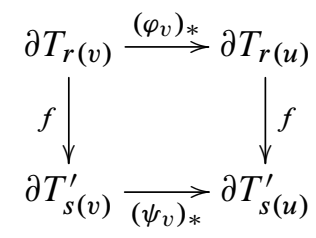

Now since $f^{-1}\left(\partial T_{v}^{\prime}\right) \subseteq \partial T_{r(v)}$, we know that

$$
\partial T_{v}^{\prime} \subseteq f\left(\partial T_{r(v)}\right) \subseteq \partial T_{s(v)}^{\prime}
$$

for each $v$. Since $T^{\prime}$ is branching, it follows that $v$ is a descendant of $s(v)$. Then, $\psi_{v}(v)$ is some descendant of $s(u)$. We claim that the set

$$
\left\{\psi_{v}(v) \mid v \in E\right\}
$$

is finite and that $f^{-1}$ has equivalent restrictions at $v, w \in E$ whenever $\psi_{v}(v)=\psi_{w}(w)$. This will prove that $f^{-1}$ has only finitely many different restrictions on $E$.

To prove that there are only finitely many possibilities for $\psi_{v}(v)$, recall that $r(v)$ is the deepest parent of $f^{-1}\left(\partial T_{v}^{\prime}\right)$ in $T$. But,

$$
\varphi_{v}(r(v))=r(u)
$$

and

$$
\left(\varphi_{v}\right)_{*}\left(f^{-1}\left(\partial T_{v}^{\prime}\right)\right)=f^{-1}\left(\left(\psi_{v}\right)_{*}\left(\partial T_{v}^{\prime}\right)\right)=f^{-1}\left(\partial T_{\psi_{v}(v)}^{\prime}\right),
$$

so $r(u)$ must be the deepest parent of $f^{-1}\left(\partial T_{\psi_{v}(v)}^{\prime}\right)$ in $T$, i.e. $r\left(\psi_{v}(v)\right)=r(u)$. But since $f^{-1}$ is continuous, it is uniformly continuous with respect to the standard ultrametrics on $\partial T^{\prime}$ and $\partial T$. In particular, there exists a $k>0$ such that

$$
d(v, w) \leq \frac{1}{2^{k}} \quad \Rightarrow \quad d\left(f^{-1}(v), f^{-1}(w)\right)<\frac{1}{2^{|r(u)|}}
$$

for all $v, w$ in $T^{\prime}$. It follows that $|r(v)|>|r(u)|$ whenever $|v| \geq k$, so there are only finitely many vertices $v$ for which $r(v)=r(u)$. Thus there are only finitely many possibilities for $\psi_{v}(v)$. 
Now suppose that $v, w \in E$ and $\psi_{v}(v)=\psi_{w}(w)$. Then $\psi_{w}^{-1} \psi_{v}$ maps $v$ to $w$, so let $\chi: T_{v} \rightarrow T_{w}$ be the restriction of this morphism. Then $\chi_{*}$ agrees with $\left(\psi_{w}\right)_{*}^{-1}\left(\psi_{v}\right)_{*}$ on $\partial T_{v}^{\prime}$, and in particular the following diagram commutes:

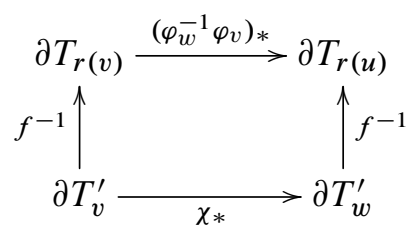

We conclude that $f^{-1}$ has equivalent restrictions at $v$ and $w$.

Corollary 2.15. If $T$ is a branching self-similar tree, then the set of all rational homeomorphisms of $\partial T$ forms a group under composition.

This is the rational group associated with $T$, denoted $R_{T}$. The next two subsections are devoted to proving the following theorem.

Theorem 2.16. Let $T$ be a branching self-similar tree. Then the associated rational group $\mathbb{R}_{T}$ is isomorphic to the binary rational group $\mathbb{R}_{2}$. Indeed, the action of $\mathbb{R}_{T}$ on $\partial T$ is conjugate to the action of $\mathbb{R}_{2}$ on $\{0,1\}^{\omega}$.

\subsection{Rigid structures.}

Definition 2.17. Let $T$ be a self-similar tree. A rigid structure for $T$ is a family $\left\{\varphi_{v w}\right\}$ of morphisms, with one morphism $\varphi_{v w}: T_{v} \rightarrow T_{w}$ for each pair $(v, w)$ of vertices of $T$ of the same type, satisfying the following conditions:

(1) $\varphi_{v w} \varphi_{u v}=\varphi_{u w}$ for all triples $(u, v, w)$ of the same type.

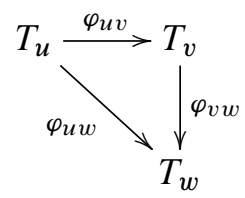

(2) If $v^{\prime}$ is a descendant of $v$ and $w^{\prime}=\varphi_{v w}\left(v^{\prime}\right)$, then $\varphi_{v^{\prime} w^{\prime}}$ is the restriction of $\varphi_{v w}$ to $T_{v^{\prime}}$.

Note that if $\left\{\varphi_{v w}\right\}$ is a rigid structure for $T$ then $\varphi_{v v} \varphi_{v v}=\varphi_{v v}$ for any vertex $v$ of $T$, and hence $\varphi_{v v}$ is the identity isomorphism of $T_{v}$. It follows that $\varphi_{w v}=\varphi_{v w}^{-1}$ for all pairs $(v, w)$.

Proposition 2.18. Every self-similar tree has a rigid structure.

Proof. Let $T$ be a self-similar tree. Choose a set $Q$ of vertices of $T$ that contains the root vertex and has exactly one vertex of each type. If $v$ is a vertex of $T$, a marking of $v$ will be an element of $\operatorname{Mor}(v, a)$, where $a$ is the vertex in $Q$ having the same type as $v$. 
Let $B$ be the set of all vertices that are children of vertices in $Q$. Choose a marking $\tau_{b}$ for each vertex $b \in \mathbb{B}$. We now define a marking $\psi_{v}$ for each vertex $v \in T$ inductively as follows:

(1) If $r$ is the root of $T$, then $\psi_{r}$ is the identity isomorphism of $T$.

(2) If $v$ is a vertex of $T$ with marking $\psi_{v}$ and $w$ is a child of $v$, let $b=\psi_{v}(w)$ denote the corresponding child of $\psi_{v}(v)$, and let

$$
\psi_{w}=\tau_{b} \psi_{v},
$$

where the composition on the right is partial, as described in Note 2.2

For each pair of vertices $(v, w)$ of $T$ of the same type, let $\varphi_{v w}=\psi_{w}^{-1} \psi_{v}$. We claim that $\left\{\varphi_{v w}\right\}$ is a rigid structure for $T$.

Clearly $\varphi_{v w} \varphi_{u v}=\varphi_{u w}$ for every triple $(u, v, w)$ of vertices of the same type. For restrictions, suppose that $v$ and $w$ have the same type and $v^{\prime}$ is a child of $v$. Let $w^{\prime}=\varphi_{v w}\left(v^{\prime}\right)$. Let $a=\psi_{v}(v)=\psi_{w}(w)$, and let $b=\psi_{v}\left(v^{\prime}\right)=\psi_{w}\left(w^{\prime}\right)$. Then

$$
\varphi_{v^{\prime} w^{\prime}}=\psi_{w^{\prime}}^{-1} \psi_{v^{\prime}}=\psi_{w}^{-1} \tau_{b}^{-1} \tau_{b} \psi_{v}=\psi_{w}^{-1} i_{b} \psi_{v}=\psi_{w}^{-1} \psi_{v} i_{v^{\prime}}=\varphi_{v w} i_{v^{\prime}},
$$

where each $i_{x}$ denotes the identity map on $T_{x}$, and we are again using partial compositions. We conclude that $\varphi_{v^{\prime} w^{\prime}}$ is the restriction of $\varphi_{v w}$ to $T_{v^{\prime}}$, as desired.

Note that a rigid structure is itself a self-similar structure on $T$. If $T$ is a selfsimilar tree and $\left\{\varphi_{v w}\right\}$ is a rigid structure on $T$, the corresponding rigid tree is the self-similar tree having the same underlying graph as $T$ but with $\left\{\varphi_{v w}\right\}$ as its self-similar structure.

Proposition 2.19. Let $T$ be a branching self-similar tree, let $\left\{\varphi_{v w}\right\}$ be a rigid structure on $T$, and let $T^{\prime}$ be the corresponding rigid tree. Then the rational homeomorphisms of $\partial T$ are the same as the rational homeomorphisms of $\partial T^{\prime}$.

Proof. Note that the identity map $i: \partial T^{\prime} \rightarrow \partial T$ is rational. In particular, for any vertices $v, w \in T^{\prime}$ of the same type, we have a commutative diagram

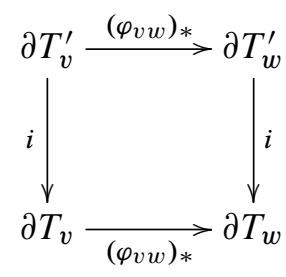

and therefore $i$ has equivalent restrictions at $v$ and $w$. Conjugating by $i$, we deduce that any rational homeomorphism of $\partial T$ is also a rational homeomorphism of $\partial T^{\prime}$, and vice versa. 
2.4. Rigid trees and $R_{2}$. In this subsection we complete the proof of Theorem 2.16 . Definition 2.20. Let $T$ and $T^{\prime}$ be self-similar trees and let $\Phi: T \rightarrow T^{\prime}$ be an isomorphism of rooted trees. We say that $\Phi$ is an isomorphism of self-similar trees if the following conditions are satisfied:

(1) Two vertices $v, w \in T$ have the same type if and only $\Phi(v)$ and $\Phi(w)$ have the same type in $T^{\prime}$.

(2) For every pair $v, w$ of vertices of $T$ of the same type, $\Phi$ conjugates $\operatorname{Mor}(v, w)$ to $\operatorname{Mor}(\Phi(v), \Phi(w))$.

For the following theorem, we say that a directed multigraph $\Gamma$ is branching if each vertex in $\Gamma$ has at least two outgoing edges.

Proposition 2.21. Let $T$ be a branching, rigid self-similar tree. Then the type graph $\Gamma$ for $T$ is branching, and there exists an isomorphism of self-similar trees $\Phi: T \rightarrow \mathcal{L}\left(\Gamma, t_{0}\right)$, where $t_{0}$ is the type of the root vertex in $T$

Proof. Since $T$ is branching, $\Gamma$ must be branching as well. Let $v_{0}$ be the root of $T$, and let $V$ be a set of vertices in $T$ that contains the root and has exactly one vertex of each type. Let $\tau: T \rightarrow V$ be the function that assigns to each vertex $x \in T$ the vertex $\tau(x) \in V$ having the same type as $x$. Then we can think of the elements of $V$ as the vertices of $\Gamma$, with one directed edge $e_{w}$ in $\Gamma$ from $v$ to $\tau(w)$ for each $v \in V$ and each child $w$ of $v$.

For any two vertices $x, y \in T$ of the same type, let $\psi_{x, y}$ denote the morphism from $x$ to $y$. Define a tree isomorphism $\Phi: \mathcal{L}\left(\Gamma, v_{0}\right) \rightarrow T$ inductively by $\Phi(\varepsilon)=v_{0}$ and

$$
\Phi\left(p e_{w}\right)=\psi_{v, \Phi(p)}(w)
$$

for each path $p$ in $\Gamma$ from $v_{0}$ to $v$ and each edge $e_{w}$ starting at $v$. Note that $\tau(\Phi(p))$ is always the endpoint of $p$, and therefore two paths $p, q$ in $\mathcal{L}\left(\Gamma, v_{0}\right)$ have the same type if and only if $\Phi(p)$ and $\Phi(q)$ have the same type in $T$.

Now let $p$ and $p^{\prime}$ be paths in $\Gamma$ from $v_{0}$ to some vertex $v \in V$, and let $\varphi_{p, p^{\prime}} \in$ $\operatorname{Mor}\left(p, p^{\prime}\right)$ be the prefix replacement, i.e.

$$
\varphi_{p, p^{\prime}}(p q)=p^{\prime} q
$$

We claim that

$$
\psi_{\Phi(p), \Phi\left(p^{\prime}\right)}(\Phi(p q))=\Phi\left(\varphi_{p, p^{\prime}}(p q)\right)
$$

for every path $q$ in $\Gamma$ starting at $v$. We proceed by induction on $q$. Note that the statement is trivially true for $q=\varepsilon$. Now suppose it is true for some path $q$ from $v$ to $v^{\prime}$, and let $e_{w}$ be an edge in $\Gamma$ starting at $v^{\prime}$. Then,

$$
\psi_{\Phi(p), \Phi\left(p^{\prime}\right)}\left(\Phi\left(p q e_{w}\right)\right)=\psi_{\Phi(p), \Phi\left(p^{\prime}\right)}\left(\psi_{v^{\prime}, \Phi(p q)}(w)\right) .
$$

But $\psi_{\Phi(p), \Phi\left(p^{\prime}\right)}(\Phi(p q))=\Phi\left(\varphi_{p, p^{\prime}}(p q)\right)=\Phi\left(p^{\prime} q\right)$, so since $T$ is rigid

$$
\psi_{\Phi(p), \Phi\left(p^{\prime}\right)} \circ \psi_{v^{\prime}, \Phi(p q)}=\psi_{v^{\prime}, \Phi\left(p^{\prime} q\right)} .
$$


Now,

$$
\psi_{\Phi(p), \Phi\left(p^{\prime}\right)}\left(\Phi\left(p q e_{w}\right)\right)=\psi_{v^{\prime}, \Phi\left(p^{\prime} q\right)}(w)=\Phi\left(p^{\prime} q e_{w}\right)=\Phi\left(\varphi_{p, p^{\prime}}\left(p q e_{w}\right)\right) .
$$

We conclude that $\Phi$ is an isomorphism of self-similar trees.

Proposition 2.22. Let $\Gamma=(V, E)$ be a finite, directed, branching multigraph, and let $v_{0} \in V$. Then there exists a rational homeomorphism from $\mathcal{L}\left(\Gamma, v_{0}\right)$ to the Cantor set $\{0,1\}^{\omega}$.

Proof. For each vertex $v \in V$, choose a complete binary prefix code for the edges of $E$ with initial vertex $v$. Together, these codes define an encoding function $c: E \rightarrow\{0,1\}^{*}$, which we can extend to a function $c: E^{*} \rightarrow\{0,1\}^{*}$ by

$$
c\left(e_{1} e_{2} \cdots e_{n}\right)=c\left(e_{1}\right) \cdot c\left(e_{2}\right) \cdots c\left(e_{n}\right) .
$$

Let $f: \partial \mathcal{L}\left(\Gamma, v_{0}\right) \rightarrow\{0,1\}^{\omega}$ be the function

$$
f\left(e_{1} e_{2} \cdots\right)=c\left(e_{1}\right) \cdot c\left(e_{2}\right) \cdots .
$$

By construction $f$ is bijective. We claim that $f$ is rational.

Let $p$ and $q$ be any two directed paths in $\Gamma$ from $v_{0}$ to the same vertex $w$, and let $\varphi_{p q}: \mathcal{L}\left(\Gamma, v_{0}\right)_{p} \rightarrow \mathcal{L}\left(\Gamma, v_{0}\right)_{q}$ be the prefix replacement morphism. Let $\varphi_{c(\alpha), c(\beta)}:\{0,1\}_{c(p)}^{*} \rightarrow\{0,1\}_{c(q)}^{*}$ be the prefix replacement morphism between the corresponding subtrees of $\{0,1\}^{*}$. Then it is easy to check that the diagram

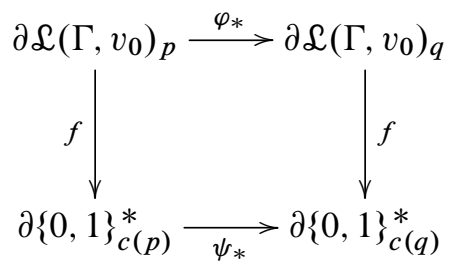

commutes. Since $\mathcal{L}\left(\Gamma, v_{0}\right)$ has only finitely many different types, this proves that $f$ is rational.

Proof of Theorem 2.16. Let $T$ be a branching self-similar tree. By Proposition 2.18, there exists a rigid structure on $T$, giving us a rigid tree $T^{\prime}$. By Proposition 2.19, we know that $\mathbb{R}_{T}=\mathbb{R}_{T}^{\prime}$. By Proposition 2.21, there exists a finite, directed, branching multigraph $\Gamma=(V, E)$ and a vertex $v_{0} \in V$ so that $T^{\prime}$ is isomorphic to $\mathcal{L}\left(\Gamma, v_{0}\right)$. Let $\Phi: T^{\prime} \rightarrow \mathcal{L}\left(\Gamma, v_{0}\right)$ an isomorphism of self-similar trees, and let $\Phi_{*}: \partial T^{\prime} \rightarrow \partial \mathcal{L}\left(\Gamma, v_{0}\right)$ be the associated rational homeomorphism. By Proposition 2.22, there exists a rational homeomorphism $h: \mathcal{L}\left(\Gamma, v_{0}\right) \rightarrow\{0,1\}^{\omega}$. Thus, the mapping

$$
f \mapsto h \circ \Phi \circ f \circ \Phi^{-1} \circ h^{-1}
$$

is an isomorphism from $\mathbb{R}_{T}$ to $\mathbb{R}_{2}$. Indeed, $h \circ \Phi$ conjugates the action of $\mathbb{R}_{T}$ on $\partial T$ to the action of $\mathbb{R}_{2}$ on $\{0,1\}^{\omega}$. 
2.5. Non-branching trees. In this section we deal with the case of non-branching self-similar trees. Note that such trees may have isolated points in their boundaries.

Definition 2.23. A self-similar tree $T$ is without dead ends if every vertex of $T$ has at least one child.

We wish to extend our theory of rational homeomorphisms to self-similar trees without dead ends. Specifically, we wish to prove that the set $R_{T}$ of rational homeomorphisms for such a tree forms a group, and that this group embeds into the binary rational group $R_{2}$.

Definition 2.24. Let $T$ be a tree without dead ends. A subtree $T_{v} \subseteq V$ is called an isolated branch if:

(1) $\partial T_{v}$ is a single point, and

(2) there does not exist an ancestor $w$ of $v$ such that $\partial T_{v}=\partial T_{w}$.

As long as $v$ is not the root, condition (2) is equivalent to saying that the parent of $v$ has at least two children. Note that isolated branches of $T$ are in one-to-one correspondence with isolated points in $\partial T$.

Definition 2.25. Let $T$ be a tree without dead ends. The expansion of $T$ is the tree $E[T]$ obtained by replacing each isolated branch $T_{v}$ of $T$ by a copy of the infinite binary tree $\{0,1\}^{*}$.

More formally, each vertex of $E[T]$ is either:

(1) a vertex $v \in T$ that does not lie in an isolated branch, or

(2) a pair $(v, \alpha)$, where $T_{v}$ is an isolated branch of $T$ and $\alpha \in\{0,1\}^{*}$.

Vertices of the first type are called old vertices, and vertices of the second type are new vertices. Descendants in $E[T]$ are defined as follows:

(1) If $v$ is an old vertex, then the descendants of $v$ in $E[T]$ consist of all old vertices that are descendants of $v$ in $T$, together with all new vertices $(w, \alpha)$ for which $w$ is a descendant of $v$ in $T$.

(2) The descendants of a new vertex $(v, \alpha)$ are all pairs $(v, \beta)$ for which $\alpha$ is a prefix of $\beta$.

Note then that each isolated branch $T_{v}$ in $T$ has a corresponding infinite binary tree $E[T]_{(v, \varepsilon)}$ of new vertices in $E[T]$.

We place a self-similar structure on $E[T]$ as follows:

(1) If $v$ and $w$ are old vertices in $E[T]$, then $v$ and $w$ have the same type in $E[T]$ if and only if they have the same type in $T$. For each morphism $\varphi: T_{v} \rightarrow T_{w}$, there is a corresponding morphism $E[\varphi]: E[T]_{v} \rightarrow E[T]_{w}$, which maps each old vertex $v^{\prime} \in E[T]_{v}$ to $\varphi\left(v^{\prime}\right)$, and maps each new vertex $\left(v^{\prime}, \alpha\right) \in E[T]_{v}$ to $\left(\varphi\left(v^{\prime}\right), \alpha\right)$. 
(2) Any two new vertices $(v, \alpha)$ and $(w \beta)$ in $E[T]$ have the same type, with a unique morphism $\varphi_{(v, \alpha),(w, \beta)}: E[T]_{(v, \alpha)} \rightarrow E[T]_{(w, \beta)}$ defined by

$$
\varphi_{(v, \alpha),(w, \beta)}(v, \alpha \psi)=(w, \beta \psi)
$$

for all $\psi \in\{0,1\}^{*}$.

It is easy to check that this satisfies the axioms for a self-similar structure.

Now, observe that each point in $\partial E[T]$ consists of either:

(1) a non-isolated point in $\partial T$, or

(2) a pair $(p, \psi)$, where $p$ is an isolated point in $\partial T$ and $\psi \in\{0,1\}^{\omega}$.

If $f: \partial T \rightarrow \partial T^{\prime}$ is a homeomorphism, then $f$ must map isolated points of $\partial T$ to isolated points of $\partial T^{\prime}$, and therefore $f$ induces a homeomorphism

$$
E[f]: \partial E[T] \rightarrow \partial E\left[T^{\prime}\right]
$$

defined by $E[f](p)=f(p)$ if $p$ is a non-isolated point in $\partial T$, and $E[f](p, \psi)=$ $(f(p), \psi)$ if $p$ is an isolated point in $\partial T$ and $\psi \in\{0,1\}^{\omega}$.

Note that this operation satisfies

$$
E[f \circ g]=E[f] \circ E[g]
$$

for any homeomorphisms $g: \partial T \rightarrow \partial T^{\prime}$ and $f: \partial T^{\prime} \rightarrow \partial T^{\prime \prime}$. Similarly, $E\left[f^{-1}\right]=$ $E[f]^{-1}$ for any homeomorphism $f: \partial T \rightarrow \partial T^{\prime}$.

Lemma 2.26. Let $T$ and $T^{\prime}$ be self-similar trees without dead ends, and let $f: \partial T \rightarrow \partial T^{\prime}$ be a homeomorphism. Then $f$ is rational if and only if the induced homeomorphism $E[f]: E[T] \rightarrow E\left[T^{\prime}\right]$ is rational.

Proof. Let $v$ and $w$ be old vertices of $E[T]$ of the same type. Since $\partial T_{v}$ and $\partial T_{w}$ each have at least two points, the images $f\left(\partial T_{v}\right)$ and $f\left(\partial T_{w}\right)$ each have at least two points, so they have deepest parents $x$ and $y$, respectively. These must be old vertices of $E\left[T^{\prime}\right]$, and indeed are the deepest parents of $E[f]\left(\partial E[T]_{v}\right)$ and $E[f]\left(\partial E[T]_{w}\right)$, respectively. Then for any two morphisms $\varphi: T_{v} \rightarrow T_{w}$ and $\psi: T_{x}^{\prime} \rightarrow T_{y}^{\prime}$, the diagram

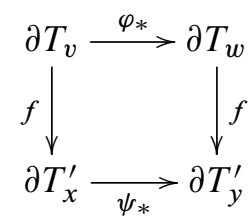

commutes if and only if the diagram

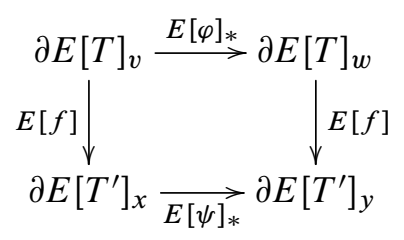

commutes, so $f$ has equivalent restrictions at $v$ and $w$ if and only if $E[f]$ has equivalent restrictions at $v$ and $w$. 
Finally, if $(v, \alpha)$ and $(w, \beta)$ are new vertices of $E[T]$, then the diagram

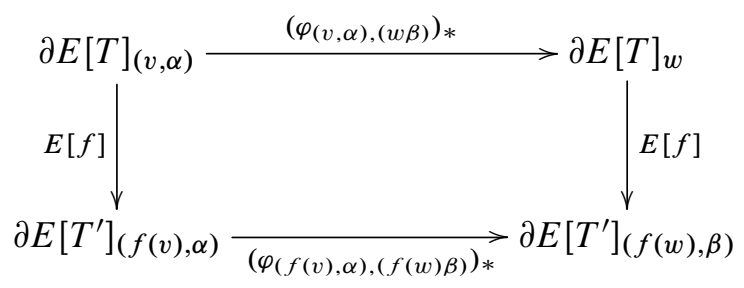

commutes, so $E[f]$ has equivalent restrictions at $(v, \alpha)$ and $(w, \beta)$. It follows that $f$ is rational if and only if $E[f]$ is rational.

Lemma 2.26 allows us to eliminate isolated branches from any tree without dead ends. That is, we can now assume that our tree $T$ without dead ends also has no isolated branches. For such a tree, any vertex with only one child must eventually have a descendant with two or more children.

Definition 2.27. Let $T$ be a self-similar tree without dead ends or isolated branches.

(1) A vertex $v \in T$ is essential if $v$ has at least two children.

(2) The simplification of $T$ is the tree $S[T]$ consisting of all essential vertices of $T$.

That is, $S[T]$ is the tree of all essential vertices of $T$, where $v$ is a descendant of $w$ in $S[T]$ if and only if $v$ is a descendant of $w$ in $T$. Note then that an essential vertex $w$ is a child in of an essential vertex $v$ in $S[T]$ if and only if $w$ is a descendant of $v$ in $T$ and each intermediate vertex on the path from $v$ to $w$ in $T$ has only one child. Since each vertex in $S[T]$ has the same number of children as in $T$, the tree $S[T]$ is branching.

We can put a self similar structure on $S[T]$ by simply restricting morphisms of $T$. That is, if $v$ and $w$ are essential vertices in $T$, then $v$ and $w$ have the same type in $S[T]$ if and only if they have the same type in $T$. Given any morphism $\varphi: T_{v} \rightarrow T_{w}$, we define a morphism $S[\varphi]: S[T]_{v} \rightarrow S[T]_{w}$ obtained from $\varphi$ by restricting to the essential vertices.

It is not hard to see that $\partial S[T]$ is naturally homeomorphic to $\partial T$, since an infinite descending path in $T$ is completely determined by which essential vertices it passes through. If $f: \partial T \rightarrow \partial T^{\prime}$ is a homeomorphism, we let $S[f]: \partial S[T] \rightarrow \partial S\left[T^{\prime}\right]$ be the induced homeomorphism.

Lemma 2.28. Let $T$ and $T^{\prime}$ be self-similar trees without isolated branches, and let $f: \partial T \rightarrow \partial T^{\prime}$. Then $f$ is rational if and only if the induced homeomorphism $S[f]: \partial S[T] \rightarrow \partial S\left[T^{\prime}\right]$ is rational.

Proof. We claim first that, if $v$ and $w$ are essential vertices of $T$ of the same type, then $f$ has equivalent restrictions at $v$ and $w$ if and only if $S[f]$ has equivalent restrictions at $v$ and $w$. To see this, let $x$ and $y$ be the deepest parents of $f\left(\partial T_{v}\right)$ and $f\left(\partial T_{w}\right)$, respectively, which exist since $T$ and $T^{\prime}$ have no isolated branches. Note that $x$ and $y$ must be essential, since otherwise they would not be deepest parents. 
Moreover, $x$ and $y$ are the deepest parents of $S[f]\left(\partial S[T]_{v}\right)$ and $S[f]\left(\partial S[T]_{w}\right)$, respectively. Then for any two morphisms $\varphi: T_{v} \rightarrow T_{w}$ and $\psi: T_{x}^{\prime} \rightarrow T_{y}^{\prime}$, the diagram

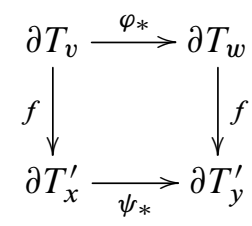

commutes if and only if the diagram

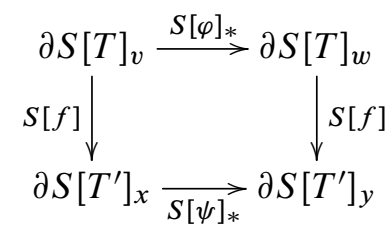

commutes, which proves the claim.

It follows immediately that $S[f]$ is rational whenever $f$ is rational. For the converse, suppose that $S[f]$ is rational. For each $v \in T$, let $r(v)$ denote the deepest parent of $\partial T_{v}$, i.e. the first descendant of $v$ that is essential. Note then that $\partial T_{r(v)}=\partial T_{v}$ for each $v$, since every infinite descending path in $T$ that passes through $v$ must pass through $r(v)$ as well. Put an equivalence relation $\sim$ on the vertices of $T$ by $v \sim w$ if $v$ and $w$ have the same type and $f$ has equivalent restrictions at $r(v)$ and $r(w)$. Since $S[f]$ is rational, we know from the previous paragraph that $f$ has only finitely many equivalence classes of restrictions at essential vertices, which means that $\sim$ has only finitely many equivalence classes. Let $E$ be such an equivalence class. It suffices to prove that $f$ has only finitely many equivalence classes of restrictions on $E$.

Fix a vertex $u \in E$. For each $v \in E$, let $s(v)$ denote the deepest parent of $f\left(\partial T_{v}\right)$, and let $\varphi_{v}: T_{r(u)} \rightarrow T_{r(v)}$ and $\psi_{v}: T_{s(u)} \rightarrow T_{s(v)}$ be morphisms that make the following diagram commute:

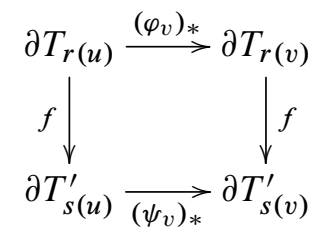

Choose also for each $v \in E$ a morphism $\chi_{v}: T_{v} \rightarrow T_{u}$. Since $\chi_{v}$ is a tree isomorphism, it must map $r(v)$ to $r(u)$ and hence $T_{r(v)}$ to $T_{r(u)}$. Then the composition $\chi_{v} \varphi_{v}$ is a morphism $T_{r(u)} \rightarrow T_{r(u)}$. We claim that $f$ has equivalent restrictions at two vertices $v, w \in E$ whenever $\chi_{v} \varphi_{v}=\chi_{w} \varphi_{w}$. Since there are only 
finitely many morphisms $T_{r(u)} \rightarrow T_{r(u)}$ in $T$, it will follow from this that $f$ has only finitely many equivalence classes of restrictions on $E$, and therefore $f$ is rational.

Let $v, w \in E$ and suppose that $\chi_{v} \varphi_{v}=\chi_{w} \varphi_{w}$. Then we have a commutative diagram

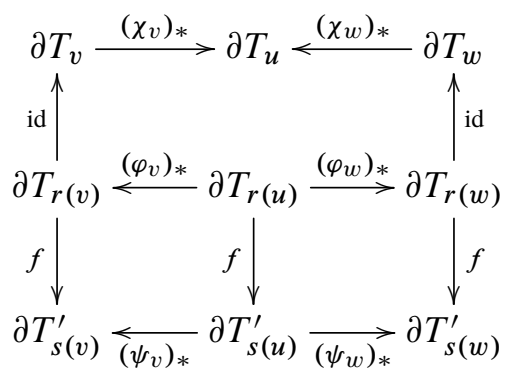

In particular, the outer square

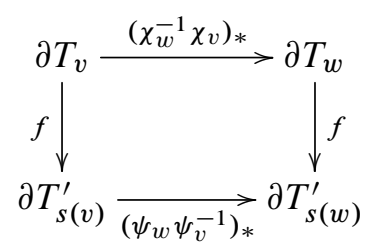

commutes, so $f$ has equivalent restrictions at $v$ and $w$.

This immediately gives us the following result in the case that there are no isolated branches.

Corollary 2.29. Let $T$ be a self-similar tree without dead ends or isolated branches. Then the set $\mathbb{R}_{T}$ of rational homeomorphisms of $T$ forms a group under composition, and this group isomorphic to $\mathbb{R}_{2}$. Indeed, the action of $\mathbb{R}_{T}$ on $\partial T$ is conjugate to the action of $\mathbb{R}_{2}$ on $\{0,1\}^{\omega}$.

Proof. By Lemma 2.28, the rational homeomorphisms of $T$ are the same as the rational homeomorphisms of $S[T]$. But $S[T]$ is branching, so the conclusion follows from Theorem 2.16.

For a tree that does have isolated branches, we get the following result.

Theorem 2.30. Let $T$ be a self-similar tree without dead ends. Then the set $\mathbb{R}_{T}$ of rational homeomorphisms of $T$ forms a group under composition. Moreover, the action of $\mathbb{R}_{T}$ on $\partial T$ is rational in the sense of Definition 2, and in particular $\mathbb{R}_{T}$ embeds into $R_{2}$.

Proof. From Lemma 2.26, we know that a homeomorphism $f: \partial T \rightarrow \partial T$ is rational if and only if the induced homeomorphism $E[f]: \partial E[T] \rightarrow \partial E[T]$ is rational. Moreover, note that $E[f g]=E[f] E[g]$ for all $f, g \in \mathbb{R}_{T}$ and $E\left[f^{-1}\right]=E[f]^{-1}$ 
for all $f \in \mathbb{R}_{T}$. Since the rational homeomorphisms of $E[T]$ form a group by Corollary 2.29, it follows that the rational homeomorphisms of $T$ form a group as well.

Now clearly the homomorphism $f \mapsto E[f]$ is an embedding of $\mathbb{R}_{E[T]}$ into $\mathbb{R}_{T}$, and since $R_{E[T]}$ is isomorphic to $R_{2}$ by Corollary 2.29 it follows that $R_{T}$ embeds into $\mathbb{R}_{2}$. Moreover, Corollary 2.29 tells us that the action of $\mathbb{R}_{E[T]}$ on $\partial E[T]$ is conjugate to the action of $R_{2}$ on $\{0,1\}^{\omega}$, so we can use $R_{E[T]}$ instead of $R_{2}$ to prove that the action of $R_{T}$ on $\partial T$ is rational. Let $q: \partial E[T] \rightarrow \partial T$ be the quotient map which is the identity on the non-isolated points of $\partial T$ and maps $\{p\} \times\{0,1\}^{\omega}$ to $p$ for each isolated point $p$ of $T$. Then clearly the diagram

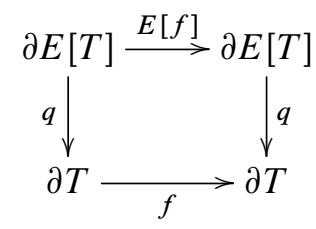

commutes for each $f \in \mathbb{R}_{T}$, and this proves that the action of $\mathbb{R}_{T}$ on $\partial T$ is rational in the sense of Definition 2.

Note that $R_{T}$ need not be isomorphic to $R_{2}$ if $T$ has isolated branches. For example, if $T$ is a self-similar tree whose boundary $\partial T$ consists of finitely many isolated points, then $R_{T}$ is a finite symmetric group.

Theorem 2.30 gives us the following test for whether an action is rational, which we use in Section 3 to prove Theorem 1.30, and hence Theorems 1 and 3.

Corollary 2.31. Let $G$ be a group acting on a compact metrizable space $X$, and let $T$ be a self-similar tree without dead ends. Suppose there exists a quotient map $q: \partial T \rightarrow X$ and a homomorphism $\varphi: G \rightarrow \mathbb{R}_{T}$ such that the diagram

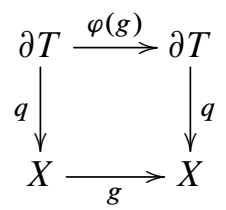

commutes for all $g \in G$. Then the action of $G$ on $X$ is rational.

\section{Rational actions of hyperbolic groups}

Let $\Gamma$ be a locally finite, connected hyperbolic graph, and let $G$ be a group acting properly and cocompactly by isometries on $\Gamma$. For example, $G$ could be any hyperbolic group and $\Gamma$ could be its Cayley graph. In this section, we construct a selfsimilar tree $T$ whose boundary $\partial T$ is naturally homeomorphic to the horofunction 
boundary $\partial_{h} \Gamma$, and we show that the action of $G$ on $\partial_{h} \Gamma$ induces a rational action of $G$ on $\partial T$.

As in Section 1, we will identify $\Gamma$ with its set of vertices under the path metric $d$. We fix a base vertex $x_{0} \in \Gamma$, and define the length $\ell(x)$ of any vertex $x \in \Gamma$ to be its distance from the base vertex. Let

$$
B_{n}=\{x \in \Gamma \mid \ell(x) \leq n\} \quad \text { and } \quad S_{n}=\{x \in \Gamma \mid \ell(x)=n\}
$$

denote the $n$-ball and $n$-sphere, respectively, centered at $x_{0}$.

If $x \in S_{n}$, a successor to $x$ is any element of $S_{n+1} \cap C(x)$, where $C(x)$ denotes the cone on $x$, and a predecessor for $x$ is any element of $S_{n-1}$ for which $x$ is a successor.

\subsection{The tree of atoms.}

Definition 3.1. Let $B$ be a finite set of vertices in $\Gamma$.

(1) Given any point $x \in \Gamma$, the corresponding atom is the set

$$
A=\left\{y \in \Gamma \mid \bar{d}_{y} \text { agrees with } \bar{d}_{x} \text { on } B\right\} .
$$

Let $Q(B)$ be the collection of all such atoms.

(2) The shape algebra for $B$, denoted $\delta(B)$, is the algebra of sets generated by $Q(B)$.

\section{Notes 3.2.}

(1) The atoms in $Q(B)$ form a partition of $\Gamma$, and therefore each element of $\delta(B)$ is a disjoint union of atoms. Thus the elements of $Q(B)$ are precisely the atoms in the Boolean algebra $S(B)$.

(2) If $B \subseteq B^{\prime}$ are finite sets, then each atom for $B^{\prime}$ is a subset of some atom for $B$. It follows that $\delta(B) \subseteq \delta\left(B^{\prime}\right)$.

Each atom $A$ comes with a function $\bar{d}_{A} \in \bar{F}(B, \mathbb{Z})$, which agrees with $\bar{d}_{x}$ on $B$ for each $x \in A$. We refer to this as the distance function for $\boldsymbol{A}$.

For example, Figure 5(a) shows the four atoms derived from a certain three-point subset of a hyperbolic graph. The corresponding shape algebra has 16 different sets, namely all possible disjoint unions of these four atoms. Figure 5(b) shows the distance function for each of the four atoms.

Proposition 3.3. Each finite set $B \subseteq \Gamma$ has only finitely many different atoms.

Proof. Fix a point $p \in B$. By the triangle inequality, we know that

$$
\left|\bar{d}_{x}(q)-\bar{d}_{x}(p)\right| \leq d(p, q)
$$

for all $x \in \Gamma$ and $q \in B$. But there are only finitely many different functions $f: B \rightarrow \mathbb{Z}$ satisfying $f(p)=0$ and

$$
|f(q)| \leq d(p, q)
$$

for all $q \in B$, and therefore there can be only finitely many atoms 


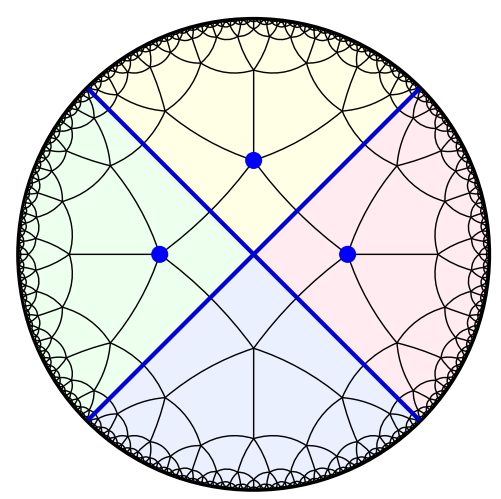

(a)

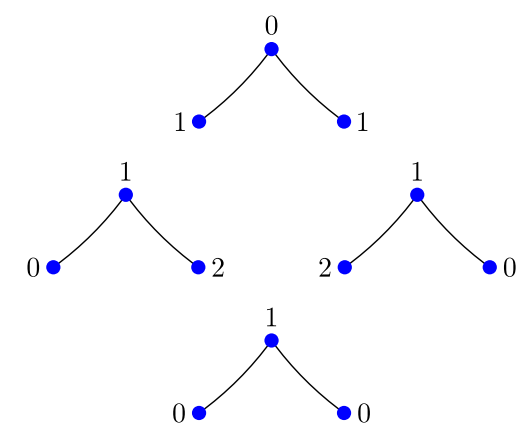

(b)

Figure 5. (a) Four atoms derived from a three-point subset $B$ of a hyperbolic graph $\Gamma$. Each atom consists of the vertices of $\Gamma$ that lie in the shown region. (b) The distance functions $\bar{d}_{A}$ for $A \in Q(B)$, with additive constants chosen so that the minimum value is 0 for each.

Now if we fix a base vertex $x_{0} \in \Gamma$, we get a sequence of balls in $\Gamma$ :

$$
\left\{x_{0}\right\}=B_{0} \subseteq B_{1} \subseteq B_{2} \subseteq \cdots
$$

Taking the corresponding atoms gives us a sequence of partitions of $\Gamma$ :

$$
Q\left(B_{0}\right), \quad Q\left(B_{1}\right), \quad Q\left(B_{2}\right), \quad \ldots
$$

Each of these partitions is a refinement of the previous one, with $Q\left(B_{0}\right)$ having only one atom, namely the whole graph $\Gamma$. For example, Figure 6 shows the atoms of $Q\left(B_{1}\right)$ and $Q\left(B_{2}\right)$ for the 1-skeleton of the order five square tiling of the hyperbolic plane.

Because each $Q\left(B_{n}\right)$ is a refinement of the previous, the disjoint union

$$
\coprod_{n=0}^{\infty} Q\left(B_{n}\right)
$$

has the structure of a rooted tree. It turns out that the boundary of this tree is naturally homeomorphic to $\Gamma \cup \partial_{h} \Gamma$, where $\partial_{h} \Gamma$ is the horofunction boundary of $\Gamma$. That is, the boundary is homeomorphic to the closure of $i(\Gamma)$ in $\bar{F}(\Gamma, \mathbb{Z})$ (see Definitions 1.22 and 1.23).

Because we are interested in $\partial_{h} \Gamma$ specifically, we would like to restrict to a subtree whose boundary is precisely $\partial_{h} \Gamma$. As we will see, it suffices to consider only the atoms in each $Q\left(B_{n}\right)$ that have infinite cardinality. This motivates the following definition. 

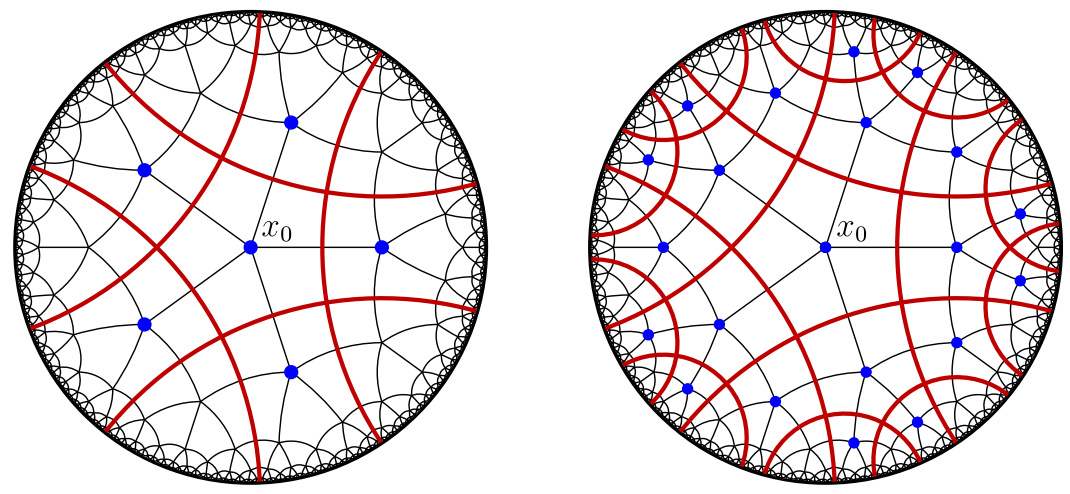

Figure 6. The atoms of $Q\left(B_{1}\right)$ and $Q\left(B_{2}\right)$ for the order five square tiling of the hyperbolic plane. There are 11 atoms in $Q\left(B_{1}\right)$ and 36 atoms in $Q\left(B_{2}\right)$.

Definition 3.4. For each $n \geq 0$, let $Q_{n}(\Gamma)$ be the set of infinite atoms in $Q\left(B_{n}\right)$. The tree of atoms for $\Gamma$ is the disjoint union.

$$
Q(\Gamma)=\coprod_{n=0}^{\infty} Q_{n}(\Gamma) .
$$

Note that, since $Q(\Gamma)$ is defined as a disjoint union, an element of $Q(\Gamma)$ is technically an ordered pair $(n, A)$, where $n \geq 0$ and $A \in Q_{n}(\Gamma)$. This distinction is sometimes relevant, for it is possible for the same set $A$ to be an atom in $Q_{n}(\Gamma)$ for two different values of $n$. However, we will often abuse notation and treat elements of $Q(\Gamma)$ as subsets of $\Gamma$, with the understanding that each atom $A \in Q(\Gamma)$ knows which $Q_{n}(\Gamma)$ it comes from.

Note also that the tree $Q(\Gamma)$ has no dead ends. In particular, since the union of the atoms in $Q_{n}(\Gamma)$ is the complement of a finite set in $\Gamma$ and every atom in $Q_{n}(\Gamma)$ is infinite, each atom in $Q_{n}(\Gamma)$ must contain at least one atom from $Q_{n+1}(\Gamma)$.

The following proposition tells us that the atoms of $Q_{n}(\Gamma)$ move away from any finite set as $n \rightarrow \infty$.

Proposition 3.5. If $n \geq 1$, then each one-point subset of $B_{n-1}$ is an atom in $Q\left(B_{n}\right)$. Thus every atom of $Q_{n}(\Gamma)$ is contained in the complement of $B_{n-1}$.

Proof. We say that a function $f \in F(\Gamma, \mathbb{Z})$ has a local minimum at a point $p \in \Gamma$ if $f(p)<f(q)$ for every vertex $q$ that is adjacent to $p$. Note that adding an arbitrary constant to $f$ does not change the positions of the local minima, so it makes sense to talk about local minima for an element $\bar{f} \in \bar{F}(\Gamma, \mathbb{Z})$.

Now, for any $x \in \Gamma$ it is not hard to see that the distance function $\bar{d}_{x}$ has a local minimum of $x$, and this is the only local minimum for $\bar{d}_{x}$ on $\Gamma$. If $x, y \in \Gamma$ 
and $x \neq y$, it follows that $\bar{d}_{x}$ and $\bar{d}_{y}$ cannot agree on any subset of $\Gamma$ that contains $x$ and all of its neighbors. In particular, if $x \in B_{n-1}$, then $B_{n}$ contains $x$ and all of its neighbors, so $\bar{d}_{x}$ does not agree with $\bar{d}_{y}$ on $B_{n}$ for any $y \neq x$, which proves that $\{x\}$ is an atom in $Q\left(B_{n}\right)$.

We will prove the following theorem in the next section.

Theorem 3.6. The boundary of the tree of atoms $Q(\Gamma)$ is naturally homeomorphic to the horofunction boundary $\partial_{h} \Gamma$ of $\Gamma$.

After this is done, the next task is to endow the tree $Q(\Gamma)$ with a self-similar structure. First, if $A \in Q_{n}(\Gamma)$ and $m \geq n$, let

$$
Q_{m}(A)=\left\{A^{\prime} \in Q_{m}(\Gamma) \mid A^{\prime} \subseteq A\right\} .
$$

The union

$$
Q(\Gamma)_{A}=\coprod_{m \geq n} Q_{m}(A)
$$

is the subtree of atoms rooted at $A$.

Definition 3.7. Let $A_{1} \in Q_{m}(\Gamma)$ and $A_{2} \in Q_{n}(\Gamma)$ be atoms. We say that an element $g \in G$ induces a morphism from $A_{1}$ to $A_{2}$ if the following conditions are satisfied:

(1) $g A_{1}=A_{2}$.

(2) $g\left(A_{1} \cap B_{m+k}\right)=A_{2} \cap B_{n+k}$ for all $k \geq 0$.

(3) For each $k>0$ and each $A_{1}^{\prime} \in Q_{m+k}\left(A_{1}\right)$, there exists an $A_{2}^{\prime} \in Q_{n+k}\left(A_{2}\right)$ such that $g A_{1}^{\prime}=A_{2}^{\prime}$.

The corresponding morphism is the isomorphism $\varphi: Q(\Gamma)_{A_{1}} \rightarrow Q(\Gamma)_{A_{2}}$ of subtrees defined by condition (3). We say that $A_{1}$ and $A_{2}$ have the same type if there exists a morphism from $Q(\Gamma)_{A_{1}}$ to $Q(\Gamma)_{A_{2}}$.

Notes 3.8.

(1) Condition (2) is equivalent to saying that

$$
\ell(g p)-n=\ell(p)-m
$$

for all $p \in A_{1}$.

(2) Clearly the composition of two morphisms is a morphism, and the inverse of a morphism is a morphism.

Proposition 3.9. For all $A_{1}, A_{2} \in Q(\Gamma)$, there are only finitely many morphisms $Q(\Gamma)_{A_{1}} \rightarrow \mathbb{Q}(\Gamma)_{A_{2}}$.

Proof. It suffices to prove that there are only finitely many morphisms from an atom $A \in Q(\Gamma)$ to itself. Let $S$ be the set of elements of $A$ of minimum length (i.e. minimum distance to the base vertex). If $\varphi: Q(\Gamma)_{A} \rightarrow Q(\Gamma)_{A}$ is a morphism corresponding to an element $g \in G$, then it follows from condition (2) that $g S=S$. Since $S$ is finite and $G$ acts properly on $\Gamma$, there are only finitely many such $g$, and therefore only finitely many morphisms from $A$ to $A$. 
We will say that two atoms $A_{1}, A_{2} \in Q(\Gamma)$ have the same type if there exists a morphism $Q(\Gamma)_{A_{1}} \rightarrow Q(\Gamma)_{A_{2}}$. Unfortunately, it is not easy to prove that there are only finitely many types of atoms in $Q(\Gamma)$, and indeed this is the first part of our theory that requires $\Gamma$ to be hyperbolic. After developing some geometric machinery in Sections 3.3 and 3.4, we prove in Section 3.5 that $Q(\Gamma)$ has only finitely many types, thereby endowing $Q(\Gamma)$ with a self-similar structure.

Finally, we prove the following theorem in Section 3.6.

Theorem 3.10. The group $G$ acts on the boundary of $Q(\Gamma)$ by rational homeomorphisms.

By Theorem 3.6 and Corollary 2.31, it follows that $G$ acts rationally on the horofunction boundary $\partial_{h} \Gamma$ (Theorem 1.30) and hence the Gromov boundary $\partial \Gamma$ (Theorem 3), and if this action is faithful then $G$ embeds in $R$ (Theorem 1). Therefore, we will have all of our chief results upon completing the proof of Theorem 3.10.

3.2. Infinite atoms and the horofunction boundary. We begin by associating to each atom a certain subset of the horofunction boundary.

Definition 3.11. Let $B \subseteq \Gamma$ be finite. Given any atom $A \in Q(B)$, the shadow of $A$ is the set

$$
\partial A=\left\{\bar{f} \in \partial_{h} \Gamma \mid \bar{f} \text { agrees with } \bar{d}_{A} \text { on } B\right\} .
$$

The following proposition explains our interest in infinite atoms.

Proposition 3.12. Let $B \subseteq \Gamma$ be a finite set, and let $A \in Q(B)$. Then the shadow $\partial A$ is nonempty if and only if $A$ is infinite.

Proof. Suppose first that $\partial A$ is nonempty, and let $\bar{f} \in \partial A$. Then by Proposition 1.27, there exist infinitely many points $x \in \Gamma$ such that $\bar{d}_{x}$ agrees with $\bar{f}$ on $B$, and it follows that $A$ is infinite.

Conversely, suppose that $A$ is infinite. Let

$$
B=B_{0} \subseteq B_{1} \subseteq B_{2} \subseteq \cdots
$$

be an ascending chain of finite sets whose union is $\Gamma$. Then we can find a descending chain

$$
A=A_{0} \supseteq A_{1} \supseteq A_{2} \supseteq \cdots,
$$

where each $A_{n}$ is an infinite atom in $Q\left(B_{n}\right)$. Then each $\bar{d}_{A_{n}}$ is a restriction of the next, and their union is a function $\bar{f} \in \bar{F}(\Gamma, \mathbb{Z})$. Clearly $\bar{f}$ agrees with $\bar{d}_{A}$ on $B$. We claim that $f$ is a horofunction.

Let $B^{\prime} \subseteq \Gamma$ be any finite set. Since $\bigcup_{n} B_{n}=\Gamma$, there exists an $n \in \mathbb{N}$ so that $B^{\prime} \subseteq B_{n}$. Since $A_{n}$ is infinite, there exist infinitely many points $x$ for which $\bar{d}_{x}$ agrees with $\bar{f}$ on $B_{n}$, and it follows from Proposition 1.27 that $f$ is a horofunction. 
Proposition 3.13. Let $B \subseteq \Gamma$ be finite. Then the sets

$$
\{\partial A \mid A \in Q(B) \text { and } A \text { is infinite }\}
$$

are a partition of $\partial_{h} \Gamma$ into clopen sets.

Proof. Since $\bar{d}_{A}$ and $\bar{d}_{A^{\prime}}$ disagree on $B$ for any two $A, A^{\prime} \in Q(B)$, the corresponding shadows are disjoint, and we know from Proposition 3.12 that they are all nonempty. To prove that the union of the shadows is all of $\partial_{h} \Gamma$, let $f: \Gamma \rightarrow \mathbb{Z}$ be a horofunction. By Proposition 1.27, there exists an $x \in \Gamma$ such that $\bar{d}_{x}$ agrees with $\bar{f}$ on $B$. Let $A \in Q(B)$ be the atom containing $x$. Then $\bar{d}_{x}$ agrees with $\bar{d}_{A}$ on $B$, so $\bar{f}$ agrees with $\bar{d}_{A}$ on $B$, and therefore $\bar{f} \in \partial A$. Finally, observe that each $\partial A$ is open in $\partial_{h} \Gamma$, since the preimage in $F(\Gamma, \mathbb{Z})$ is open in the product topology. Since there are only finitely many $\partial A$, it follows that each $\partial A$ is also closed.

Proof of Theorem 3.6. To show that $\partial Q(\Gamma)$ is homeomorphic to $\partial_{h} \Gamma$, we begin by defining a function $h: \partial_{h} \Gamma \rightarrow \partial Q(\Gamma)$ as follows. Given a point $\bar{f} \in \partial_{h} \Gamma$, we know from Proposition 3.13 that there exists for each $n \in \mathbb{N}$ an atom $A_{n} \in Q_{n}(\Gamma)$ whose shadow contains $\bar{f}$. Then for each $n$, the distance functions $\bar{d}_{A_{n}}$ and $\bar{d}_{A_{n+1}}$ must both agree with $\bar{f}$ on $B_{n}$, and therefore $\bar{d}_{A_{n}}$ is the restriction to $B_{n}$ of $\bar{d}_{A_{n+1}}$. It follows that the sequence $\left\{A_{n}\right\}$ is nested, with $A_{0} \supseteq A_{1} \supseteq \cdots$, so it corresponds to an infinite descending path in the tree $Q(\Gamma)$. Let $h(\bar{f})$ be this path. Note that $h$ is continuous since the sets $\left\{\partial Q(\Gamma)_{A} \mid A \in Q(\Gamma)\right\}$ form a basis for the topology on $\partial Q(\Gamma)$, and $h^{-1}\left(\partial Q(\Gamma)_{A}\right)=\partial A$ is open in $\partial_{h} \Gamma$ for each $A$.

To prove that $h$ is bijective, let

$$
A_{0} \supseteq A_{1} \supseteq A_{2} \supseteq \cdots .
$$

be any infinite descending path in $Q(\Gamma)$, where $A_{n} \in Q_{n}(\Gamma)$ for each $n$. Then $\bar{d}_{A_{n+1}}$ agrees with $\bar{d}_{A_{n}}$ on $B_{n}$ for each $n$, and therefore

$$
\partial A_{0} \supseteq \partial A_{1} \supseteq \partial A_{2} \supseteq \cdots .
$$

Since each $\partial A_{n}$ is closed and $\partial_{h} \Gamma$ is compact, the intersection $\bigcap_{n=0}^{\infty} \partial A_{n}$ contains at least one point. This maps to $\left\{A_{n}\right\}$ under $h$, and therefore $h$ is surjective. Moreover, if $f$ and $f^{\prime}$ are horofunctions such that $\bar{f}, \bar{f}^{\prime} \in \bigcap_{n=0}^{\infty} A_{n}$, then for each $n$ both $\bar{f}$ and $\bar{f}^{\prime}$ agree with $\bar{d}_{A_{n}}$ on $B_{n}$, and therefore $\bar{f}$ and $\bar{f}^{\prime}$ agree with each other on $B_{n}$. Since this holds for every $n$, it follows that $\bar{f}=\bar{f}^{\prime}$, so the intersection $\bigcap_{n=0}^{\infty} A_{n}$ is a single point. This proves that $h$ is injective and hence bijective. Since $\partial_{h} \Gamma$ and $\partial Q(\Gamma)$ are compact Hausdorff spaces, it follows that $h$ is a homeomorphism.

3.3. Nearest neighbors and visibility. In this section we develop some geometric tools that will be essential in our proofs. 


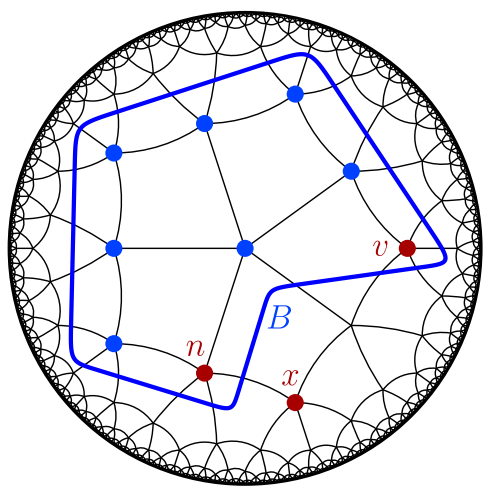

Figure 7. A point $x$ and a finite subset $B$ of a hyperbolic graph $\Gamma$. Here $N(x, B)=\{n\}$ and $V(x, B)=\{n, v\}$. In particular, $n$ is a nearest neighbor to $x$, while $v$ is visible from $x$ but is not a nearest neighbor.

Definition 3.14. Let $B \subseteq \Gamma$ be a finite set, and let $x \in \Gamma$.

(1) A point $p \in B$ is called a nearest neighbor for $x$ if

$$
d(p, x) \leq d(q, x)
$$

for all $q \in B$. We let $N(x, B)$ denote the set of nearest neighbors to $x$ in $B$.

(2) A point $p \in B$ is visible from $x$ if

$$
d(p, x)<d(p, q)+d(q, x)
$$

for all $q \in B \backslash\{p\}$. We let $V(x, B)$ denote the set of points in $B$ that are visible from $x$.

Equivalently, a point $p \in B$ is visible from $x$ if

$$
[p, x] \cap B=\{p\}
$$

for every geodesic $[p, x]$ from $p$ to $x$.

Clearly $N(x, B) \subseteq V(x, B)$, i.e. every nearest neighbor to $x$ in $B$ is visible from $x$. However, there are sometimes points in $B$ that are visible from $x$ but are not nearest neighbors, as shown in Figure 7.

Proposition 3.15. Let $B \subseteq \Gamma$ be a finite set, and let $x, y \in \Gamma$ be points that lie in the same atom of $Q(B)$. Then,

$$
N(x, B)=N(y, B) \quad \text { and } \quad V(x, B)=V(y, B) .
$$

Proof. For the first statement, observe that a point $p \in B$ lies in $N(x, B)$ if and only if

$$
d_{x}(q)-d_{x}(p) \geq 0
$$


for all $q \in B$. But $\bar{d}_{x}$ and $\bar{d}_{y}$ agree on $B$, so

$$
d_{x}(q)-d_{x}(p)=d_{y}(q)-d_{y}(p)
$$

for all $x, y \in B$, which implies that $N(x, B)=N(y, B)$.

A similar argument holds for $V(x, B)$. Specifically, a point $p \in B$ lies in $V(x, B)$ if and only if

$$
d_{x}(p)-d_{x}(q)<d(p, q)
$$

for all $q \in B$. Again, the quantity on the left depends only on $\bar{d}_{x}$, so it follows that $V(x, B)=V(y, B)$.

If $B \subseteq \Gamma$ is a finite set and $A \in Q(B)$ we will let

$$
N(A, B) \text { and } V(A, B)
$$

denote the set of points in $B$ that are nearest neighbors to points in $A$ and visible from points in $A$, respectively. That is, $N(A, B)=N(x, B)$ and $V(A, B)=V(x, B)$ for all $x \in A$. This is well-defined by Proposition 3.15.

Though nearest neighbors are fairly natural, visibility may see like a strange concept. The following proposition explains our interest in visibility.

Proposition 3.16. Let $B \subseteq \Gamma$ be a finite set, let $p \in B$, and let $x \in \Gamma$. Then there exists a geodesic from $p$ to $x$ that contains a point of $V(x, B)$.

Proof. Let $\left(q_{1}, \ldots, q_{n}\right)$ be any geodesic from $q_{1}=p$ to $q_{n}=x$. Let $q_{i}$ be the last point in this geodesic that lies in $B$. If $q_{i}$ is visible from $x$ we are done. Otherwise, we can replace $\left(q_{i}, q_{i+1}, \ldots, q_{n}\right)$ with another geodesic from $q_{i}$ to $x$ that intersects $B$ at a later point. Continuing in this fashion, we eventually obtain a geodesic that contains a point of $V(x, B)$.

The proposition above is actually the defining property of $V(x, B)$. Specifically, if $B \subseteq \Gamma$ is a finite set, $x \in \Gamma$, and $P \subseteq B$, the following are equivalent:

(1) $V(x, B) \subseteq P$.

(2) For every point $p \in B$, every geodesic from $p$ to $x$ contains a point of $P$.

Condition (1) implies condition (2) by Proposition 3.16, while condition (2) implies condition (1) since every visible point $p \in B$ has a geodesic $[p, x]$ that intersects $B$ only at $p$.

We can use Proposition 3.16 to obtain a useful test for whether a given point lies in a given atom.

Lemma 3.17. Let $B \subseteq \Gamma$ be finite, let $x \in \Gamma$, and let $P \subseteq B$ be any set that contains $V(x, B)$. Then, for all $b \in B$,

$$
d(b, x)=\min _{p \in P}[d(b, p)+d(p, x)] .
$$


Proof. Let $b \in B$, and let $m$ be the given minimum. By the triangle inequality,

$$
d(b, x) \leq d(b, p)+d(p, x)
$$

for all $p \in P$, and therefore $d(b, x) \leq m$. For the reverse inequality, Proposition 3.16 tells us that there exists a geodesic $[b, x]$ from $b$ to $x$ that intersects $V(x, B)$. Let $p \in[b, x] \cap V(x, B)$. Then $p \in P$ and

$$
d(b, p)+d(p, x)=d(b, x),
$$

which proves that $m \leq d(b, x)$.

Proposition 3.18. Let $B \subseteq \Gamma$ be a finite set. Let $x \in \Gamma$ and $A \in Q(B)$, and let $P \subseteq B$ be any set containing $V(x, B) \cup V(A, B)$. Then $x \in A$ if and only if $\bar{d}_{x}$ agrees with $\bar{d}_{A}$ on $P$.

Proof. If $x \in A$, then $\bar{d}_{x}$ and $\bar{d}_{A}$ must agree on all of $B$, and therefore $\bar{d}_{x}$ agrees with $\bar{d}_{A}$ on $P$. For the converse, suppose that $\bar{d}_{x}$ agrees with $\bar{d}_{A}$ on $P$. Let $y \in A$, and note that $V(y, B)=V(A, B) \subseteq P$ and $\bar{d}_{x}$ and $\bar{d}_{y}$ agree on $P$. By Lemma 3.17, we know that

$$
d_{x}(b)=\min _{p \in P}\left[d(b, p)+d_{x}(p)\right] \quad \text { and } \quad d_{y}(b)=\min _{p \in P}\left[d(b, p)+d_{y}(p)\right]
$$

for all $p \in B$. Since $\bar{d}_{x}$ and $\bar{d}_{y}$ agree on $P$, it follows that $\bar{d}_{x}$ and $\bar{d}_{y}$ agree on all of $B$. Then $\bar{d}_{x}$ agrees with $\bar{d}_{A}$ on $B$, so $x \in A$.

3.4. A membership test for atoms. In this section we use the notions of nearest neighbors and visibility to obtain a useful test for whether a given point $x \in \Gamma$ lies in a given atom of $Q(\Gamma)$.

Recall that $B_{n}$ denotes the $n$-ball in $\Gamma$ centered at the base vertex $x_{0}$. Note that if $x$ is any point in $\Gamma$ with $\ell(x) \geq n$, then $N\left(x, B_{n}\right)$ and $V\left(x, B_{n}\right)$ are both subsets of the $n$-sphere $S_{n}$. If particular, $N\left(x, B_{n}\right)$ is precisely the set of points in $S_{n}$ that lie on geodesics from $x_{0}$ to $x$.

The following fundamental proposition helps us pin down the locations of the points in $V\left(x, B_{n}\right)$. Though we assumed at the beginning of Section 3 that $\Gamma$ is $\delta$-hyperbolic, the following proposition is the first time we make use of this fact.

Proposition 3.19. Let $x \in \Gamma$, let $n \leq \ell(x)$, and let $p \in V\left(x, B_{n}\right)$. Let $x_{0}, x_{1}, \ldots, x_{\ell(x)}$ be a geodesic from $x_{0}$ to $x$ and let $p_{0}, p_{1}, \ldots, p_{n}$ be a geodesic from $x_{0}$ to $p$. Then,

$$
d\left(x_{i}, p_{i}\right) \leq 4 \delta+2
$$

for all $0 \leq i \leq n$. 
Proof. Let $0 \leq i \leq n$. If $i \leq 2 \delta+1$ we are done, so suppose that $i>2 \delta+1$. Let $[p, x]$ be a geodesic from $p$ to $x$. Since $p$ is visible from $x$, this intersects $B_{n}$ only at $p$. Let $j=\min (n-1-\delta, i)$ and note that $j<n-\delta$, which means that the point $p_{j}$ does not lie within $\delta$ of any point on $[p, x]$. Since $\Gamma$ is $\delta$-hyperbolic, it follows that $d\left(p_{j}, x_{k}\right) \leq \delta$ for some $k$. Note then that $|j-k| \leq \delta$, so

$$
|i-k| \leq|i-j|+|j-k| \leq(\delta+1)+\delta=2 \delta+1 .
$$

Then,

$$
\begin{aligned}
d\left(x_{i}, p_{i}\right) & \leq d\left(x_{i}, x_{k}\right)+d\left(x_{k}, p_{j}\right)+d\left(p_{j}, p_{i}\right) \\
& \leq|i-k|+\delta+|i-j| \leq(2 \delta+1)+\delta+(\delta+1)=4 \delta+2 .
\end{aligned}
$$

This proposition motivates the following definition.

Definition 3.20. Let $x \in \Gamma$, let $n \leq \ell(x)$, and let $p \in S_{n}$. We say that $p$ is proximal to $x$ if there exists a geodesic $p_{0}, p_{1}, \ldots, p_{n}$ from $x_{0}$ to $p$ such that

$$
d\left(x_{i}, p_{i}\right) \leq 4 \delta+2
$$

for all $0 \leq i \leq n$ and every geodesic $x_{0}, x_{1}, \ldots, x_{m}$ from $x_{0}$ to $x$. We let $P\left(x, S_{n}\right)$ be the set of points in $S_{n}$ that are proximal to $x$.

By Proposition 3.19, every point in $B_{n}$ that is visible to $x$ must be proximal to $x$, i.e. $V\left(x, B_{n}\right) \subseteq P\left(x, S_{n}\right)$.

The advantage of $P\left(x, S_{n}\right)$ over $V\left(x, B_{n}\right)$ is that there is a useful inductive test to check whether a point $p \in S_{n}$ is proximal to $x$, as shown in the following proposition.

Proposition 3.21. Let $n \geq 1$, let $x \in \Gamma$ with $\ell(x) \geq n$, and let $p \in S_{n}$. Then $p$ is proximal to $x$ if and only if:

(1) $p$ has a predecessor that is proximal to $x$, and

(2) $d(p, q) \leq 4 \delta+2$ for all $q \in N\left(x, B_{n}\right)$.

Proof. Suppose that $p$ is proximal to $x$, and let $p_{0}, p_{1}, \ldots, p_{n}$ be a geodesic from $x_{0}$ to $p$ satisfying the definition of proximality. Then $p_{n-1}$ is a predecessor for $p_{n}$ and is clearly proximal, with $p_{0}, p_{1}, \ldots, p_{n-1}$ being the required geodesic. Furthermore, if $q$ is a nearest neighbor to $x$ in $B_{n}$, then there must exist a geodesic $x_{0}, \ldots, x_{\ell}(x)$ from $x_{0}$ to $x$ such that $x_{n}=q$, and it follows that

$$
d(q, p)=d\left(x_{n}, p_{n}\right) \leq 4 \delta+2 .
$$

For the converse, suppose that $p$ satisfies conditions (1) and (2). Let $p_{n-1}$ be a predecessor to $p$ that is proximal to $x$, and let $p_{0}, \ldots, p_{n-1}$ be a geodesic from $x_{0}$ to $p_{n-1}$ satisfying the definition of proximality. Note then that $p_{0}, \ldots, p_{n-1}, p$ is a geodesic from $x_{0}$ to $p$. Let $x_{0}, \ldots, x_{\ell(x)}$ be a geodesic from $x_{0}$ to $x$. We know that

$$
d\left(x_{i}, p_{i}\right) \leq 4 \delta+2
$$


for all $0 \leq i \leq n-1$. Moreover, the point $x_{n}$ must lie in $N\left(x, B_{n}\right)$, and therefore

$$
d\left(p, x_{n}\right) \leq 4 \delta+2,
$$

which proves that $p$ is proximal to $x$.

Corollary 3.22. If $x \in \Gamma$ and $n \leq \ell(x)$, then $P\left(x, S_{n}\right)$ has diameter at most $8 \delta+4$.

The proposition above showed that $P\left(x, S_{n}\right)$ is in some sense determined by nearest neighbors. The following proposition carries this idea further.

Proposition 3.23. Let $n \geq 0$, let $x, y \in \Gamma$ with $\ell(x) \geq n$ and $\ell(y) \geq n$, and suppose that $N\left(x, B_{n}\right) \subseteq N\left(y, B_{n}\right)$. Then $P\left(y, S_{n}\right) \subseteq P\left(x, S_{n}\right)$.

Proof. We use induction on $n$. The statement is obvious for $n=0$, since

$$
P\left(x, S_{0}\right)=P\left(y, S_{0}\right)=\left\{x_{0}\right\} .
$$

For $n>0$, suppose that

$$
N\left(x, B_{n}\right) \subseteq N\left(y, B_{n}\right),
$$

and let $p \in P\left(y, S_{n}\right)$. We know from Proposition 3.21 that $p$ has a predecessor $p^{\prime} \in P\left(y, S_{n-1}\right)$. But $N\left(x, B_{n-1}\right) \subseteq N\left(y, B_{n-1}\right)$, since $N\left(x, B_{n-1}\right)$ consists of all predecessors of elements of $N\left(x, B_{n}\right)$, and similarly for $y$. From our induction hypothesis, it follows that

$$
P\left(y, S_{n-1}\right) \subseteq P\left(x, S_{n-1}\right),
$$

so $p^{\prime} \in P\left(x, S_{n-1}\right)$. But,

$$
d(p, q) \leq 4 \delta+2
$$

for all $q \in N\left(y, B_{n}\right)$, and hence $d(p, q) \leq 4 \delta+2$ for all $q \in N\left(x, B_{n}\right)$, which proves that $p \in P\left(x, S_{n}\right)$ by Proposition 3.21.

Corollary 3.24. Let $n \geq 0$, and let $x, y \in \Gamma$ with $\ell(x) \geq n$ and $\ell(y) \geq n$. If $x$ and $y$ lie in the same atom of $Q\left(B_{n}\right)$, then

$$
P\left(x, S_{n}\right)=P\left(y, S_{n}\right) .
$$

Proof. By Proposition 3.15 we know that $N\left(x, B_{n}\right)=N\left(y, B_{n}\right)=N\left(A, B_{n}\right)$, so the result follows from Proposition 3.23.

If $A \in Q_{n}(\Gamma)$, we will let $P\left(A, S_{n}\right)$ denote the set of points in $S_{n}$ that are proximal to points in $A$, i.e. $P\left(A, S_{n}\right)=P\left(x, S_{n}\right)$ for any $x \in A$. This is well-defined by Corollary 3.24.

We now show how to use proximal points to determine whether a given point lies in a given atom. 
Proposition 3.25. Let $x \in \Gamma$ and $A \in Q_{n}(\Gamma)$. Then $x \in A$ if and only if:

(1) $x \in C(p)$ for all $p \in N\left(A, B_{n}\right)$, and

(2) $\bar{d}_{x}$ agrees with $\bar{d}_{A}$ on $P\left(A, S_{n}\right)$.

Proof. Clearly $x$ satisfies the given conditions if it lies in $A$. For the converse, suppose that $x$ satisfies the given conditions. Since

$$
N\left(x, B_{n}\right)=\left\{p \in S_{n} \mid x \in C(p)\right\},
$$

it follows from condition (1) that $N\left(A, B_{n}\right) \subseteq N\left(x, B_{n}\right)$, so by Proposition 3.23 we have $P\left(x, S_{n}\right) \subseteq P\left(A, S_{n}\right)$. Then,

$$
N\left(x, B_{n}\right) \subseteq V\left(x, B_{n}\right) \subseteq P\left(x, S_{n}\right) \subseteq P\left(A, S_{n}\right),
$$

so $N\left(x, B_{n}\right)=N\left(x, P\left(A, S_{n}\right)\right)$. (In general $N(x, B)=N\left(x, B^{\prime}\right)$ whenever $B^{\prime} \subseteq$ $B \subseteq \Gamma$ and $N(x, B) \subseteq B^{\prime}$.) But similarly,

$$
N\left(A, B_{n}\right) \subseteq V\left(A, B_{n}\right) \subseteq P\left(A, S_{n}\right),
$$

so $N\left(A, B_{n}\right)=N\left(A, P\left(A, S_{n}\right)\right)$. But $\bar{d}_{x}$ agrees with $\bar{d}_{A}$ on $P\left(A, S_{n}\right)$ by condition (2), which implies that

$$
N\left(x, P\left(A, S_{n}\right)\right)=N\left(A, P\left(A, S_{n}\right)\right),
$$

and hence $N\left(x, B_{n}\right)=N\left(A, B_{n}\right)$. Then $P\left(x, S_{n}\right)=P\left(A, S_{n}\right)$ by Proposition 3.23. By Proposition 3.19, this set contains $V\left(x, B_{n}\right) \cup V\left(A, B_{n}\right)$, so it follows from Proposition 3.18 that $x \in A$.

3.5. Finitely many types. In this section we exploit our membership test for atoms (Proposition 3.25) to prove that the tree $Q(\Gamma)$ has only finitely many different types of atoms. The difficult part here is to construct a sufficient number of morphisms between atoms, so the main technical result for this section is a test for whether a given element of $G$ induces a morphism between two given atoms.

First we introduce a little notation. Given a nonempty subset $P \subseteq \Gamma$, a function $f \in F(P, \mathbb{Z})$, and a group element $g \in G$, let $g f \in F(g P, \mathbb{Z})$ be the function defined by

$$
(g f)(p)=f\left(g^{-1} p\right)
$$

for all $p \in g P$. Note that if $f$ and $f^{\prime}$ differ by a constant, then $g f$ and $g f^{\prime}$ differ by a constant, so for any $\bar{f} \in \bar{F}(P, \mathbb{Z})$ we get a well-defined $g \bar{f} \in \bar{F}(g P, \mathbb{Z})$.

Definition 3.26. Let $A \in Q_{m}(\Gamma)$ and $A^{\prime} \in Q_{n}(\Gamma)$. We say that an element $g \in G$ induces a geometric equivalence from $A$ to $A^{\prime}$ if:

(1) $g P\left(A, S_{m}\right)=P\left(A^{\prime}, S_{n}\right)$,

(2) $g \bar{d}_{A}$ agrees with $\bar{d}_{A^{\prime}}$ on $P\left(A^{\prime}, S_{n}\right)$, and

(3) $g C(p)=C(g p)$ for all $p \in P\left(A, P_{m}\right)$. 
Here is the main technical result for this section:

Proposition 3.27. Let $A \in \mathfrak{Q}_{m}(\Gamma)$ and $A^{\prime} \in Q_{n}(\Gamma)$, and let $g \in G$. If $g$ induces a geometric equivalence from $A$ to $A^{\prime}$, then $g$ induces a morphism from $A$ to $A^{\prime}$.

As we will see in Section 4 (Note 4.1), the converse of this proposition is not true, so this test does not allow us to detect all morphisms of $Q(\Gamma)$. However, it does provide us with a sufficient number of morphisms to prove the following result.

Corollary 3.28. The tree $Q(\Gamma)$ has finitely many types of atoms.

Proof. Since $P\left(A, S_{m}\right)$ has diameter at most $8 \delta+4$ (Corollary 3.28), there are only finitely many possibilities for $P\left(A, S_{m}\right)$ modulo the action of $G$. Moreover, since the action of $G$ is proper and $\Gamma$ has finitely many cone types, there are only finitely many choices for $C(p)$ for each $p \in P\left(A, S_{m}\right)$, and there are only finitely many choices for the restriction of $\bar{d}_{A}$ to $P\left(A, S_{m}\right)$. By Proposition 3.27, two atoms with corresponding choices have the same type, and therefore there are only finitely many types of atoms.

The rest of this section is devoted to a proof of Proposition 3.27.

Lemma 3.29. Let $A \in Q_{m}(\Gamma)$ and $A^{\prime} \in Q_{n}(\Gamma)$, and let $g \in G$. If $g$ induces $a$ geometric equivalence from $A$ to $A^{\prime}$, then:

(1) $g N\left(A, B_{m}\right)=N\left(A^{\prime}, B_{n}\right)$,

(2) $g A=A^{\prime}$, and

(3) $g\left(A \cap B_{m+k}\right)=A^{\prime} \cap B_{n+k}$ for all $k \geq 0$.

Proof. For statement (1), observe that the points of $N\left(A, B_{m}\right)$ are precisely the points at which $\bar{d}_{A}$ achieves its minimum value on $B_{m}$. Indeed, since $N\left(A, B_{m}\right) \subseteq$ $P\left(A, S_{m}\right)$, the points of $N\left(A, B_{m}\right)$ are precisely the points of $P\left(A, S_{m}\right)$ on which the restriction of $\bar{d}_{A}$ achieves its minimum value. Since

$$
g P\left(A, S_{m}\right)=P\left(A^{\prime}, S_{n}\right)
$$

and $g \bar{d}_{A}$ agrees with $\bar{d}_{A^{\prime}}$ on $P\left(A^{\prime}, S_{n}\right)$, it follows that $g N\left(A, B_{m}\right)$ is precisely the set of points of $P\left(A^{\prime}, S_{n}\right)$ on which the restriction of $\bar{d}_{A^{\prime}}$ achieves its minimum value, and therefore

$$
g N\left(A, B_{m}\right)=N\left(A^{\prime}, B_{n}\right) .
$$

Statement (2) uses our membership test for atoms (Proposition 3.25). Let $x \in A$, and observe that $x \in C(p)$ for all $p \in N\left(A, B_{m}\right)$. Since $g$ is a geometric equivalence and $N\left(A, B_{m}\right) \subseteq P\left(A, S_{m}\right)$, we know that

$$
g C(p)=C(g p)
$$

for all $p \in N\left(A, B_{m}\right)$, so $g x \in C(g p)$ for all $p \in N\left(A, B_{m}\right)$. By statement (1), we know that $g N\left(A, B_{m}\right)=N\left(A^{\prime}, B_{n}\right)$, and therefore $g x \in C(q)$ for all $q \in N\left(A^{\prime}, B_{n}\right)$. 
Moreover, since $\bar{d}_{x}$ agrees with $\bar{d}_{A}$ on $P\left(A, S_{m}\right)$, we see that $\bar{d}_{g x}=g \bar{d}_{x}$ agrees with $g \bar{d}_{A}=\bar{d}_{A^{\prime}}$ on $P\left(A^{\prime}, S_{n}\right)$, and therefore $g x \in A^{\prime}$ by Proposition 3.25. This proves that $g A \subseteq A^{\prime}$, and the reverse inclusion follows from the fact that $g^{-1}$ is a geometric equivalence from $A^{\prime}$ to $A$.

For statement (3), let $k>0$ and fix any point $p \in N\left(A, B_{m}\right)$. Then $p \in N\left(x, B_{m}\right)$ for all $x \in A$, which means that for each $x \in A$ there exists a geodesic from $x_{0}$ to $x$ that goes through $p$. Since $\ell(p)=m$, it follows that

$$
A \cap B_{m+k}=\{x \in A \mid d(x, p)=k\} .
$$

Moreover, $g p \in N\left(A^{\prime}, B_{n}\right)$ by statement (1), so similarly

$$
A^{\prime} \cap B_{n+k}=\left\{y \in A^{\prime} \mid d(y, g p)=k\right\} .
$$

Since $g A=A^{\prime}$ by statement (2), it follows that $g\left(A \cap B_{m+k}\right)=A^{\prime} \cap B_{n+k}$.

Lemma 3.30. Let $p \in \Gamma$, let $p^{\prime}$ be a successor to $p$, and let $g \in G$. If $g C(p)=C(g p)$, then $g p^{\prime}$ is a successor to $g p$, and $g C\left(p^{\prime}\right)=C\left(g p^{\prime}\right)$.

Proof. Note first that, for any point $p \in \Gamma$, the set of successors to $p$ is precisely the set of points in $C(p)$ that are adjacent to $p$. Moreover, if $p^{\prime}$ is a successor to $p$, then

$$
C\left(p^{\prime}\right)=\left\{x \in C(p) \mid d(x, p)=d\left(x, p^{\prime}\right)+1\right\} .
$$

Since $G$ acts by isometries, the lemma follows immediately.

If $x \in \Gamma$ and $n \in \mathbb{N}$, let $A_{n}(x)$ denote the atom of $Q\left(B_{n}\right)$ that contains $x$. Note that

$$
A_{0}(x) \supseteq A_{1}(x) \supseteq A_{2}(x) \supseteq \cdots
$$

and that $A_{n}(x) \in Q_{n}(\Gamma)$ if and only if $A_{n}(x)$ is infinite.

Lemma 3.31. Let $x \in \Gamma$, let $m, n \geq 1$, and let $g \in G$. Suppose that $A_{m}(x)$ and $A_{n}(g x)$ are both infinite, and suppose that $g$ induces a geometric equivalence from $A_{m-1}(x)$ to $A_{n-1}(g x)$. Then $g$ induces a geometric equivalence from $A_{m}(x)$ to $A_{n}(g x)$

Proof. Let $y=g x$. Since $A_{m}(x)$ and $A_{n}(y)$ are both infinite, we know from Proposition 3.5 that $\ell(x) \geq m$ and $\ell(y) \geq n$. Let $X$ be the set of all successors of elements of $P\left(x, S_{m-1}\right)$, and let $Y$ be the set of all successors of elements of $P\left(y, S_{n-1}\right)$.

We have $g P\left(x, S_{m-1}\right)=P\left(y, S_{n-1}\right)$ as $g$ is a geometric equivalence between $A_{m-1}(x)$ and $A_{n-1}(y)$. Also, $g C(p)=C(g p)$ for every point $p \in P\left(x, S_{m-1}\right)$. By Lemma 3.30, it follows that $g X=Y$ and $g C(p)=C(g p)$ for all $p \in X$. In particular, since $P\left(x, S_{m}\right) \subseteq X$ by Proposition 3.21, we know that $g C(p)=C(g p)$ for all $p \in P\left(x, S_{m}\right)$. 
Next observe that

$$
N\left(x, B_{m}\right) \subseteq V\left(x, B_{m}\right) \subseteq P\left(x, S_{m}\right) \subseteq X,
$$

and similarly for $y$. It follows that $N\left(x, B_{m}\right)=N(x, X)$ and $N\left(y, B_{n}\right)=N(y, Y)$. Since $g x=y$ and $g X=Y$, it we conclude that $g N\left(x, B_{m}\right)=N\left(y, B_{n}\right)$. But

$$
P\left(x, S_{m}\right)=\left\{p \in X \mid d(p, q) \leq 4 \delta+2 \text { for all } q \in N\left(x, B_{m}\right)\right\}
$$

by Proposition 3.21, and similarly for $y$, so $g P\left(x, S_{m}\right)=P\left(y, S_{n}\right)$.

Finally, since $g \bar{d}_{x}=\bar{d}_{y}$ and $\bar{d}_{x}$ agrees with $\bar{d}_{A_{m}(x)}$ on $P\left(x, S_{m}\right)$ and $\bar{d}_{y}$ agrees with $\bar{d}_{A_{n}(y)}$ on $P\left(y, S_{n}\right)$, it follows that $g \bar{d}_{A_{m}(x)}$ agrees with $\bar{d}_{A_{n}(y)}$ on $P\left(y, S_{n}\right)$, and therefore $g$ is a geometric equivalence from $A_{m}(x)$ to $A_{n}(y)$.

Proof of Proposition 3.27. Let $A \in Q_{m}(\Gamma)$, let $A^{\prime} \in Q_{n}(\Gamma)$, and let $g \in G$ be a geometric equivalence from $A$ to $A^{\prime}$. We must show that $g$ induces a morphism from $Q(\Gamma)_{A}$ to $Q(\Gamma)_{A}^{\prime}$.

Note first that

$$
g A=A^{\prime} \quad \text { and } \quad g\left(A \cap B_{m+k}\right)=A^{\prime} \cap B_{n+k}
$$

for each $k \geq 0$ by Lemma 3.29. We claim that for $k \geq 0$ and each $A_{k} \in Q_{m+k}(A)$, there exists an $A_{k}^{\prime} \in Q_{n+k}\left(A^{\prime}\right)$ such that $g$ induces a geometric equivalence from $A_{k}$ to $A_{k}^{\prime}$. By Lemma 3.29, it will follow that $g A_{k}=A_{k}^{\prime}$, which will prove that $g$ induces a morphism.

We proceed by induction on $k$. We know the statement holds for $k=0$, since $A_{k}=A$ and $A_{k}^{\prime}=A^{\prime}$ in this case. For $k>0$, since $A_{k}$ is infinite and the union of the atoms in $Q_{n+k}\left(A^{\prime}\right)$ is the complement of a finite set in $A^{\prime}$, we can choose a point $x \in A_{k}$ such that $g x$ lies in some infinite atom $A_{k}^{\prime} \in Q_{n+k}\left(A^{\prime}\right)$. Let $y=g x$, and let

$$
A_{k-1}=A_{m+k-1}(x) \text {. }
$$

By our induction hypothesis, there exists an atom $A_{k-1}^{\prime} \in Q_{n+k-1}\left(A^{\prime}\right)$ such that $g$ induces a geometric equivalence from $A_{k-1}$ to $A_{k-1}^{\prime}$. By Lemma 3.29, we know that $g A_{k-1}=A_{k-1}^{\prime}$, so

$$
A_{k-1}^{\prime}=A_{n+k-1}(y) \text {. }
$$

By Lemma 3.31, we conclude that $g$ induces a geometric equivalence from

$$
A_{m+k}(x)=A_{k} \quad \text { to } \quad A_{n+k}(y)=A_{k}^{\prime},
$$

which completes the induction.

3.6. Proof of rationality. Our goal in this section is to prove Theorem 3.10, i.e. that the action of $G$ on $\partial Q(\Gamma)$ is rational.

If $g \in G$, we define the magnitude of $g$, denoted $|g|$, to be the distance from the base vertex $x_{0}$ to $g x_{0}$. For example, if $\Gamma$ is the Cayley graph of $G$, then $|g|$ is the word length of $g$. Note that $|g|=\left|g^{-1}\right|$ for all $g \in G$. 
Proposition 3.32. Let $g \in G$ with $|g|=k$, and let $A \in Q_{n}(\Gamma)$ for some $n \geq k$. Then there exists a unique $A^{\prime} \in Q_{n-k}(\Gamma)$ so that $g A \subseteq A^{\prime}$.

Proof. Observe that

$$
|\ell(g x)-\ell(x)| \leq|g|=k
$$

for all $x \in \Gamma$. It follows easily that $B_{n-k} \subseteq g B_{n}$. Since $A \in Q\left(B_{n}\right)$, we know that $g A \in Q\left(g B_{n}\right)$, and therefore $g A \subseteq A^{\prime}$ for some $A^{\prime} \in Q\left(B_{n-k}\right)$. This $A^{\prime}$ is unique since the atoms of $Q\left(B_{n-k}\right)$ are disjoint.

Note 3.33. It follows immediately from this proposition that the homeomorphism of $\partial Q(\Gamma)$ induced by an element $g \in G$ is Lipschitz with respect to the standard ultrametric, with Lipschitz constant $2^{|g|}$. Indeed, since $g^{-1}$ is Lipschitz as well, it follows that the homeomorphism induced by $g$ is biLipschitz.

Proposition 3.32 prompts the following definition.

Definition 3.34. A mapping triple is an ordered triple $\left(g, A, A^{\prime}\right)$, where

(1) $g \in G$,

(2) $A \in Q_{n}(\Gamma)$ for some $n \geq|g|$, and

(3) $A^{\prime}$ is the atom from $Q_{n-|g|}(\Gamma)$ that contains $g A$.

By Proposition 3.32, for every $g \in G$ and every $A \in Q_{n}(\Gamma)$ where $n \geq|g|$, there exists a unique $A^{\prime} \in \mathbb{Q}_{n-|g|}(\Gamma)$ such that $\left(g, A, A^{\prime}\right)$ is a mapping triple.

Proposition 3.35. Let $\left(g, A, A^{\prime}\right)$ be a mapping triple, where $|g|=k$ and $A \in Q_{n}(\Gamma)$. Then there exist points $p \in P\left(A, S_{n}\right)$ and $q \in P\left(A^{\prime}, S_{n-k}\right)$ so that $d(g p, q) \leq 2 k$.

Proof. Fix a point $x \in A$, and note that $g x \in A^{\prime}$. Since $n \geq k$, we know that $g^{-1} x_{0} \in B_{n}$. By Proposition 3.16, there exists a geodesic $\left[g^{-1} x_{0}, x\right]$ from $g^{-1} x_{0}$ to $x$ that goes through a point $p \in V\left(A, B_{n}\right)$. Let $\left[x_{0}, g x\right]$ be the image of this geodesic under $g$, and let $q \in N\left(A^{\prime}, B_{n-k}\right)$ be the point at which this geodesic crosses $S_{n}$. Note that $p \in P\left(A, S_{n}\right)$ and $q \in P\left(A^{\prime}, S_{n-k}\right)$. Since $\ell(p)=n$, we know that

$$
n-k \leq \ell(g p) \leq n+k .
$$

Since $\ell(q)=n-k$ and $g p, q \in\left[x_{0}, g x\right]$, it follows that $d(g p, q) \leq 2 k$.

If $g \in G$, let $L_{g}: \partial Q(\Gamma) \rightarrow \partial Q(\Gamma)$ be the homeomorphism induced by $g$.

Proposition 3.36. Let $\left(g, A_{1}, A_{1}^{\prime}\right)$ and $\left(g, A_{2}, A_{2}^{\prime}\right)$ be mapping triples, and suppose there exists an $h \in G$ so that:

(1) $h$ induces a morphism from $A_{1}^{\prime}$ to $A_{2}^{\prime}$, and

(2) $g^{-1} h g$ induces a morphism from $A_{1}$ to $A_{2}$.

Then $L_{g}$ has equivalent restrictions at $A_{1}$ and $A_{2}$. 
Proof. Let $\psi \in \operatorname{Mor}\left(A_{1}^{\prime}, A_{2}^{\prime}\right)$ and $\varphi \in \operatorname{Mor}\left(A_{1}, A_{2}\right)$ be the induced morphisms, and note that $\psi_{*}: \partial A_{1}^{\prime} \rightarrow \partial A_{2}^{\prime}$ is a restriction of $L_{h}$ and $\varphi_{*}: A_{1} \rightarrow A_{2}$ is a restriction of $L_{g^{-1} h g}$. Then the diagram

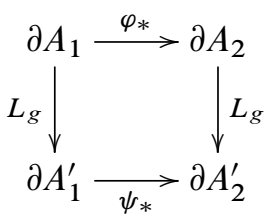

commutes since $L_{g} L_{g^{-1} h g}=L_{h} L_{g}$, so $L_{g}$ has equivalent restrictions at $A_{1}$ and $A_{2}$.

Proof of Theorem 3.10. Fix an element $g \in G$ with $|g|=k$. Given a mapping triple ( $\left.g, A, A^{\prime}\right)$ with $A \in Q_{n}(\Gamma)$, define the signature of $\left(g, A, A^{\prime}\right)$ to be the following information:

(1) The sets $g P\left(A, S_{n}\right)$ and $P\left(A^{\prime}, S_{n-k}\right)$.

(2) The functions $g \bar{d}_{A}$ on $g P\left(A, S_{n}\right)$ and $\bar{d}_{A^{\prime}}$ on $P\left(A^{\prime}, S_{n-k}\right)$.

(3) The set $g C\left(g^{-1} p\right)$ for each $p \in g P\left(A, S_{n}\right)$, and the cone $C(q)$ for each $q \in P\left(A^{\prime}, S_{n-k}\right)$.

We say that two mapping triples $\left(g, A_{1}, A_{1}^{\prime}\right)$ and $\left(g, A_{2}, A_{2}^{\prime}\right)$ with $A_{1} \in Q_{m}(\Gamma)$ and $A_{2} \in Q_{n}(\Gamma)$ have equivalent signatures if there exists an $h \in G$ so that:

(1) $h g P\left(A_{1}, S_{m}\right)=g P\left(A_{2}, S_{n}\right)$ and $h P\left(A_{1}^{\prime}, S_{m-k}\right)=P\left(A_{2}^{\prime}, S_{n-k}\right)$,

(2) $h g \bar{d}_{A_{1}}$ agrees with $g \bar{d}_{A_{2}}$ on $g P\left(A_{2}, S_{n}\right)$, and $h \bar{d}_{A_{1}^{\prime}}$ agrees with $\bar{d}_{A_{2}^{\prime}}$ on $P\left(A_{2}^{\prime}, S_{n-k}\right)$, and

(3) $h g C\left(g^{-1} p\right)=g C\left(g^{-1} h p\right)$ for all $p \in g P\left(A_{1}, S_{m}\right)$ and $h C(q)=C(h q)$ for all $q \in P\left(A_{1}^{\prime}, S_{m-k}\right)$.

By Propositions 3.27 and 3.36, if $\left(g, A_{1}, A_{1}^{\prime}\right)$ and $\left(g, A_{2}, A_{2}^{\prime}\right)$ have equivalent signatures, then $L_{g}$ has equivalent restrictions at $A_{1}$ and $A_{2}$. In particular, $h$ clearly induces a morphism from $A_{1}^{\prime}$ to $A_{2}^{\prime}$ by Proposition 3.27, and it is easy to show using Proposition 3.27 that $g^{-1} h g$ induces a morphism from $A_{1}$ to $A_{2}$.

Finally, it is not hard to see that there are only finitely many equivalence classes of signatures for a given $g \in G$. In particular, each of the sets $g P\left(A, S_{n}\right)$ and $P\left(A^{\prime}, S_{n-k}\right)$ has diameter at most $8 \delta+4$, so by Proposition 3.35 the union

$$
g P\left(A, S_{n}\right) \cup P\left(A^{\prime}, S_{n-k}\right)
$$

has diameter at most $16 \delta+8+2 k$. Thus there are only finitely many possible pairs

$$
\left(g P\left(A, S_{n}\right), P\left(A^{\prime}, S_{n-k}\right)\right)
$$

up to the action of $G$. Once such a pair is chosen, there are only finitely many possible choices for parts (2) and (3) of the signature. We conclude that $L_{g}$ has only finitely many restrictions, so $L_{g}$ is rational. 


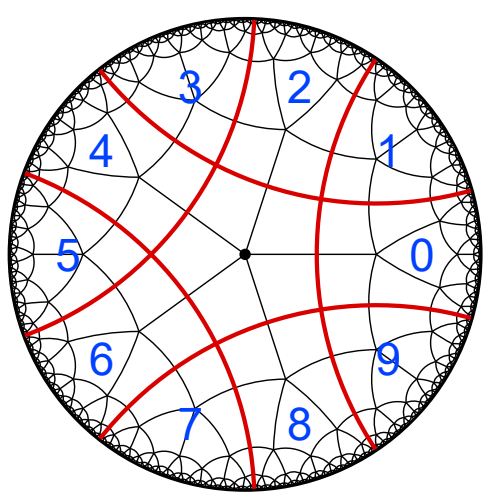

Figure 8. The atoms of $Q\left(B_{1}\right)$.

\section{An example}

In this section we work out a specific example of a tree of atoms and the corresponding rational homeomorphisms. Let $\Gamma$ be the 1-skeleton of order five square tiling of the hyperbolic plane (see Example 1.12), and fix a vertex $x_{0}$ of $\Gamma$. Let $G$ be the group of orientation-preserving isometries of $\Gamma$. We will demonstrate a rational action of $G$.

4.1. The atoms. We begin by classifying the atoms in $\Gamma$. As in any graph, the first atom is the whole graph $\Gamma$, which is the only atom of $Q_{0}(\Gamma)$, and is the root of the tree of atoms. We will refer to this atom as having type $A$.

Figure 8 shows the atoms of $Q\left(B_{1}\right)$. There is one finite atom, namely the singleton set $\left\{x_{0}\right\}$, as well as ten infinite atoms, which we have labeled with the digits $0, \ldots, 9$. As suggested by the shapes, there are two types of infinite atoms here:

- Atoms 0, 2, 4, 6, and 8 are bounded by two geodesic rays and a geodesic segment. These atoms all have the same type, which we will refer to as type $B$.

- Atoms 1, 3, 5, 7, and 9 are bounded by two geodesic rays. These atoms all have the same type, which we will refer to as type $C$.

Thus the root of the tree of atoms has type A, with ten children in $Q_{1}(\Gamma)$ of types $B$ and $\mathrm{C}$ :

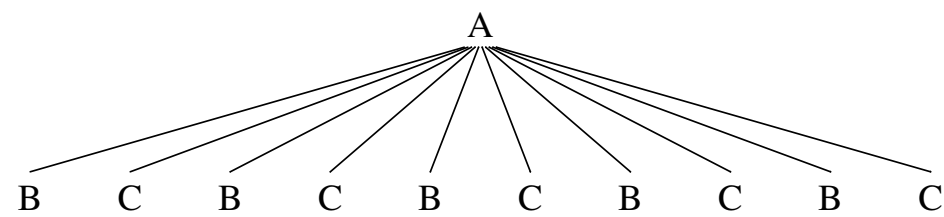

Figure 9 shows the distance functions $\bar{d}_{A}$ associated with the singleton atom $\left\{x_{0}\right\}$ and the atoms 0 and 5 . In each case, the additive constant has been chosen so that $\bar{d}_{A}$ has a minimum value of 0 on $B_{1}$. 


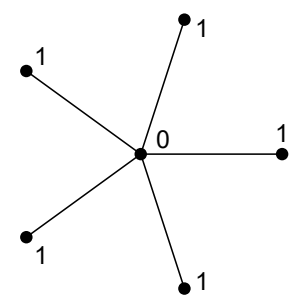

$\left\{x_{0}\right\}$

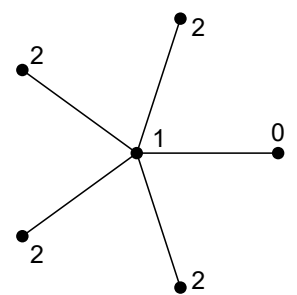

Atom 0

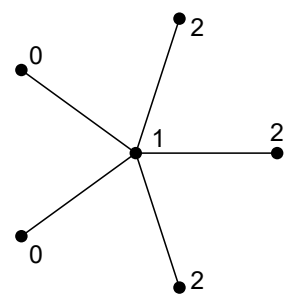

Atom 5

Figure 9. Distance functions $\bar{d}_{A}$ on $B_{1}$ for three different atoms from $Q\left(B_{1}\right)$.

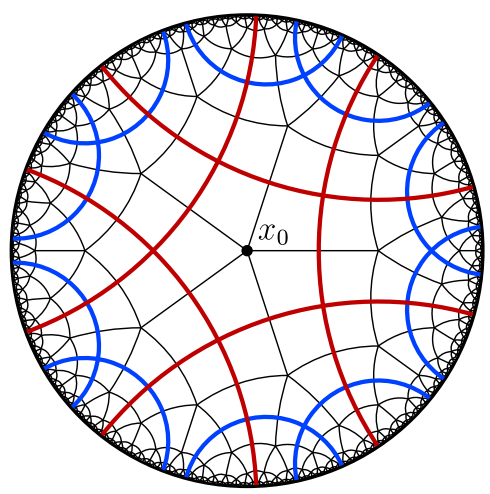

Figure 10. The atoms of $Q\left(B_{2}\right)$.

Of course, the atoms of $Q\left(B_{1}\right)$ are subdivided further in $Q\left(B_{2}\right)$. Figure 10 shows the atoms of $Q\left(B_{2}\right)$, with the new subdivisions indicated in blue. As you can see, each atom of type $B$ from $Q\left(B_{1}\right)$ has been subdivided into four atoms in $Q\left(B_{2}\right)$, and each atom of type $C$ from $Q\left(B_{1}\right)$ has been subdivided into three atoms in $Q\left(B_{2}\right)$. Thus, $Q\left(B_{2}\right)$ has a total of 36 atoms. Of these, only 30 are infinite, and therefore $Q_{2}(\Gamma)$ has 30 elements.

Figure 11 shows a close-up of atom 0 from $Q\left(B_{1}\right)$, which has type $B$, as well as the subdivision of this atom in $Q\left(B_{2}\right)$. As the figure suggests, a type $B$ atom is subdivided into one singleton atom, two atoms of type $B$, and one atom of type $C$. Thus every type B node in the tree of atoms has three children of types B, C, and B:

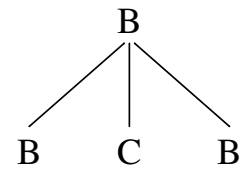



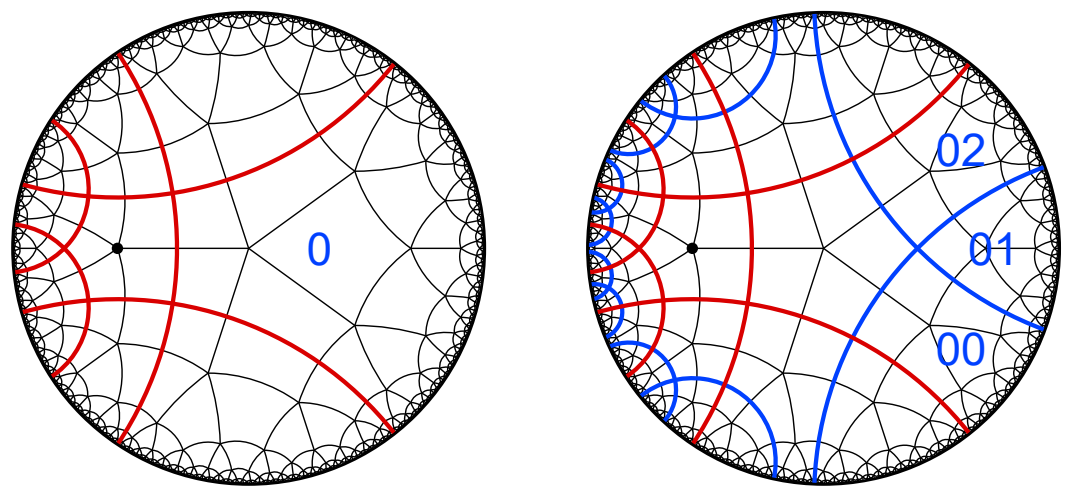

Figure 11. Subdividing an atom of type B.
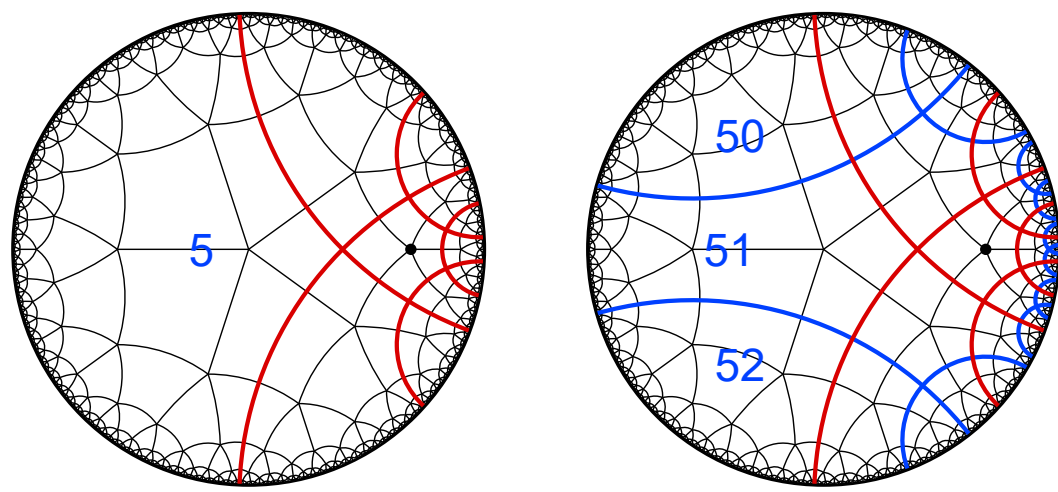

Figure 12. Subdividing an atom of type C.

Figure 12 shows a close-up of the type $C$ atom in $Q\left(B_{1}\right)$ immediately to the left of the base vertex, as well as its subdivision in $Q\left(B_{2}\right)$. As the figure suggests, a type $C$ atom is subdivided into two atoms of type $\mathrm{C}$ and one atom of a new type, which we refer to as type D. Type D atoms have a "pentagon" shape, and are bounded by two geodesic rays and two geodesic segments in the hyperbolic plane. Thus every type $\mathrm{C}$ node in the tree of atoms has three children of types C, D, and C:

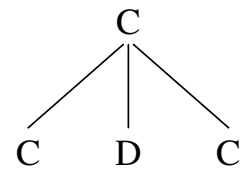




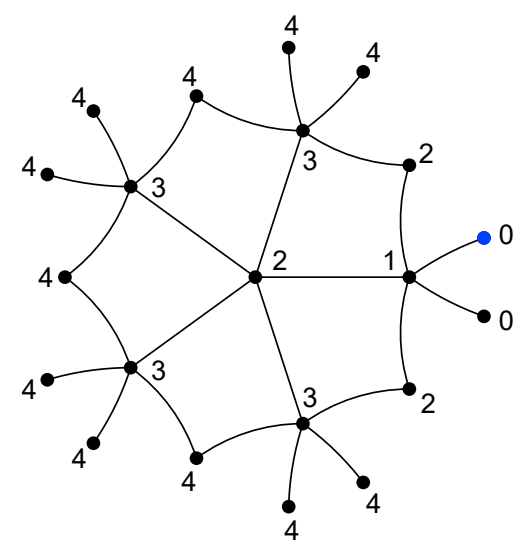

(a)

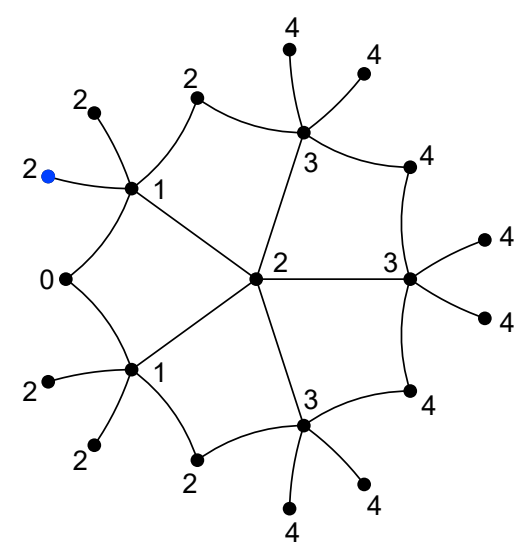

(b)

Figure 13. (a) The distance function $\bar{d}_{A}$ for atom 01 . The one for atom 00 is the same except that the value for the blue vertex changes to 2 . (b) The distance function $\bar{d}_{A}$ for atom 51 . The one for atom 50 is the same except that the value for the blue vertex changes to 0 .

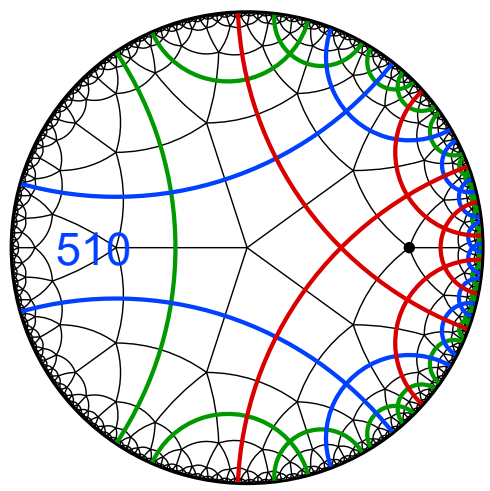

Figure 14. Subdividing an atom of type D.

Figure 13 shows the distance functions $\bar{d}_{A}$ for the atoms 01 and 51 .

Finally, Figure 14 shows the subdivision in $Q\left(B_{3}\right)$ of this same atom of type $C$. As the picture suggests, the type $D$ child atom is subdivided in the next level into a singleton atom and an atom of type B. Thus every type D node in the tree of atoms has exactly one child of type B: 


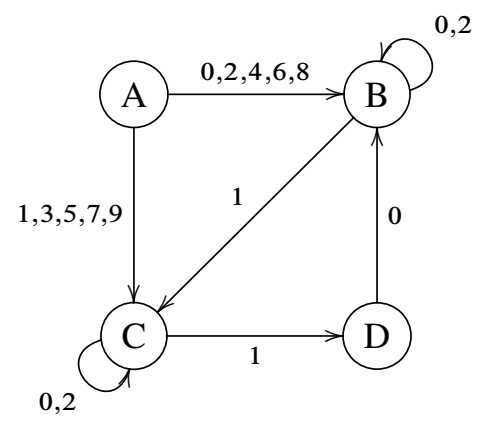

Figure 15. The type graph for the tree of atoms. Directed edges with multiple labels represent multiple edges.

The type graph for the full tree of atoms is shown in Figure 15. By Proposition 2.21, the tree $Q(\Gamma)$ is isomorphic to the set of all finite directed paths in this graph starting at $\mathrm{A}$, and $\partial_{h} \Gamma$ is naturally homeomorphic to the space of all infinite directed paths in this graph starting at $\mathrm{A}$.

Note 4.1. Not every pair of atoms of the same type in $Q(\Gamma)$ are geometrically equivalent in the sense of Definition 3.26. In particular, since $\Gamma$ is not a tree the hyperbolic constant $\delta$ is at least 1 , so $2 \delta+1 \geq 3$. Then it follows easily from the definition of proximal points (Definition 3.20) that $P\left(x, S_{n}\right)=S_{n}$ whenever $n \leq 3$, and in particular $P\left(A, S_{1}\right)=S_{1}$ for all $A \in Q_{1}(\Gamma)$ and $P\left(A, S_{2}\right)=S_{2}$ for all $A \in Q_{2}(\Gamma)$. Since $S_{1}$ has five vertices and $S_{2}$ has fifteen vertices, it follows that no atom in $Q_{1}(\Gamma)$ is geometrically equivalent to an atom in $Q_{2}(\Gamma)$, even though both $Q_{1}(\Gamma)$ and $Q_{2}(\Gamma)$ contain atoms of types $B$ and $C$.

4.2. The group. Let $G$ be the group of orientation-preserving isometries of $\Gamma$. Since we are restricting to orientation-preserving isometries, there is a unique morphism between any two atoms of the same type, so the tree of atoms is rigid.

The group $G$ has presentation

$$
\left\langle r, s \mid r^{5}, s^{2},(r s)^{4}\right\rangle,
$$

where

(1) $r$ is a counterclockwise rotation by $2 \pi / 5$ at the base vertex $x_{0}$, and

(2) $s$ is a rotation by $\pi$ at the point $p$ shown in Figure 16 .

The rational homeomorphism for $r$ is given by the formulas:

$$
\begin{array}{lllll}
r(0 \beta)=2 \beta, & r(1 \gamma)=3 \gamma, & r(2 \beta)=4 \beta, & r(3 \gamma)=5 \gamma, & r(4 \beta)=6 \beta, \\
r(5 \gamma)=7 \gamma, & r(6 \beta)=8 \beta, & r(7 \gamma)=9 \gamma, & r(8 \beta)=0 \beta, & r(9 \gamma)=1 \gamma,
\end{array}
$$

where $\beta$ can be any infinite path in the type graph starting at $\mathrm{B}$, and $\gamma$ can be any valid infinite path in the type graph starting at $\mathrm{C}$. 


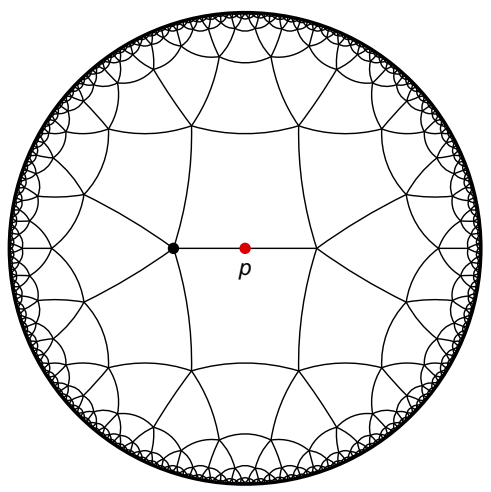

Figure 16. The group $G$ is generated by the stabilizer of $x_{0}$ and a $180^{\circ}$ rotation at the point $p$.

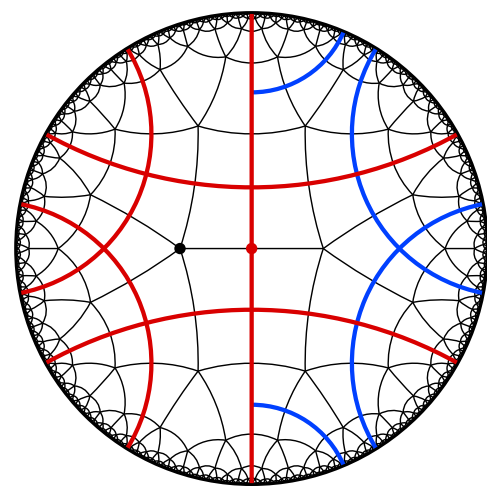

(a)

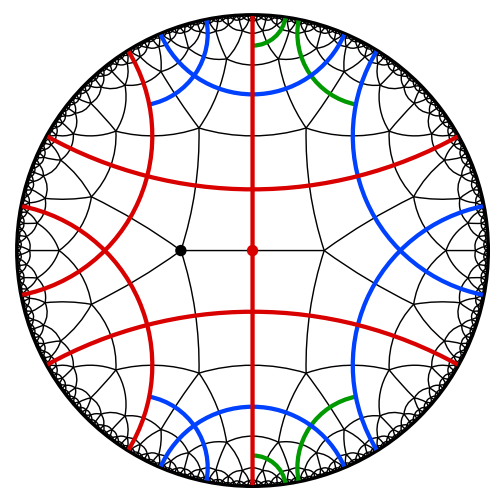

(b)

Figure 17. Two subdivisions of $\partial_{h} \Gamma$ into atoms.

The formula for $s$ is more complicated. Figure 17(a) shows a subdivision of $\Gamma$ into certain atoms of $Q\left(B_{1}\right)$ and $Q\left(B_{2}\right)$. From this subdivision, we can see that:

$$
\begin{array}{llll}
s(0 \beta)=s_{0}(\beta), & s(1 \gamma)=s_{1}(\gamma), & s(2 \beta)=9 f(\beta), & s(3 \gamma)=92 \gamma, \\
s(4 \beta)=00 \beta, & s(5 \gamma)=01 \gamma, & s(6 \beta)=02 \beta, & s(7 \gamma)=10 \gamma, \\
s(8 \beta)=1 \bar{f}(\beta), & s(9 \gamma)=s_{9}(\gamma), &
\end{array}
$$

where $\beta, \gamma$, and $\delta$ represent any infinite paths in the type graph starting at $\mathrm{B}, \mathrm{C}$, and $\mathrm{D}$, respectively, and

$$
\begin{array}{lll}
s_{0}(0 \beta)=4 \beta, & s_{0}(1 \gamma)=5 \gamma, & s_{0}(2 \beta)=6 \beta, \\
s_{1}(0 \gamma)=7 \gamma, & s_{1}(1 \delta)=8 h(\delta), & s_{1}(2 \gamma)=8 g(\gamma), \\
s_{9}(0 \gamma)=2 \bar{g}(\gamma), & s_{9}(1 \delta)=2 \bar{h}(\delta), & s_{9}(2 \gamma)=3 \gamma .
\end{array}
$$


Figure 17(b) shows a refinement of this subdivision that can be used to determine $f, \bar{f}, g, \bar{g}, h$, and $\bar{h}$ :

$$
\begin{aligned}
& f(0 \beta)=0 f(\beta), \quad f(1 \gamma)=02 \gamma, \quad f(2 \beta)=10 \beta, \\
& \bar{f}(0 \beta)=10 \beta, \quad \bar{f}(1 \gamma)=20 \gamma, \quad \bar{f}(2 \beta)=2 \bar{f}(\beta), \\
& g(0 \gamma)=1 \gamma, \quad g(1 \delta)=2 h(\delta), \quad g(2 \gamma)=2 g(\gamma), \\
& \bar{g}(0 \gamma)=0 \bar{g}(\gamma), \quad \bar{g}(1 \delta)=0 \bar{h}(\delta), \quad \bar{g}(2 \gamma)=1 \gamma, \\
& h(0 \beta)=0 \beta, \quad \bar{h}(0 \beta)=2 \beta \text {. }
\end{aligned}
$$

Each of the letters $s, s_{0}, s_{1}, s_{9}, f, \bar{f}, g, \bar{g}, h$, and $\bar{h}$ represents an equivalence class of atoms on which $s$ has equivalent restrictions, and there are also classes for the identity functions $\mathrm{id}_{B}, \mathrm{id}_{C}$, and $\mathrm{id}_{D}$ corresponding to types $\mathrm{B}, \mathrm{C}$, and $\mathrm{D}$, respectively. The atoms corresponding to each restriction type are as follows:

- $s$ : the root atom only,

- $s_{0}$ : atom 0 only,

- $s_{1}$ : atom 1 only,

- $s_{9}$ : atom 9 only,

- $f: 20^{n}$ for any $n \geq 0$,

- $\bar{f}: 12^{n}$ for any $n \geq 0$,

- $g: 12^{n}$ for any $n \geq 0$,
- $\bar{g}: 90^{n}$ for any $n \geq 0$,

- $h: 12^{n} 1$ for any $n \geq 0$,

- $\bar{h}: 90^{n} 1$ for any $n \geq 0$,

- $\operatorname{id}_{B}$ : all other type B atoms,

- $\operatorname{id}_{C}$ : all other type $\mathrm{C}$ atoms,

- $\operatorname{id}_{D}$ : all other type D atoms.

\section{References}

[1] https://mathoverflow.net/questions/328741

[2] D. Alessandrini, L. Liu, A. Papadopoulos, and W. Su, The horofunction compactification of Teichmüller spaces of surfaces with boundary, Topology Appl., 208 (2016), 160-191. Zbl 1343.32008 MR 3506976

[3] W. Ballmann, M. Gromov, and V. Schroeder, Manifolds of nonpositive curvature, Progress in Mathematics, 61, Birkhäuser Boston, Inc., Boston, MA, 1985. Zbl 0591.53001 MR 823981

[4] L. Bartholdi, V. A. Kaimanovich, and V. Nekrashevych, On amenability of automata groups, Duke Math. J., 154 (2010), no. 3, 575-598. Zbl 1268.20026 MR 2730578

[5] L. Bartholdi and Z. Šuník, Some solvable automaton groups, Topological and asymptotic aspects of group theory, 11-29, Contemp. Math., 394, Amer. Math. Soc., Providence, RI, 2006. Zbl 1106.20021 MR 2216703

[6] J. Belk and C. Bleak, Some undecidability results for asynchronous transducers and the Brin-Thompson group 2V, Trans. Amer. Math. Soc., 369 (2017), no. 5, 3157-3172. Zbl 1364.20015 MR 3605967 
[7] J. Belk, J. Hyde, and F. Matucci, On the asynchronous rational group, Groups Geom. Dyn., 13 (2019), no. 4, 1271-1284. Zbl 07154976 MR 4033505

[8] C. Bleak, P. Cameron, Y. Maissel, A. Navas, and F. Olukoya, The further chameleon groups of Richard Thompson and Graham Higman: Automorphisms via dynamics for the Higman groups $G_{n, r}$, submitted. arXiv:1605.09302v2

[9] C. Bleak, P. Cameron, and F. Olukoya, Automorphisms of shift spaces and the HigmanThompson groups: the two-sided case, 2020. arXiv:2006.01466

[10] M. R. Bridson and A. Haefliger, Metric spaces of non-positive curvature, Grundlehren der Mathematischen Wissenschaften, 319, Springer-Verlag, Berlin, 1999. Zbl 0988.53001 MR 1744486

[11] A. M. Brunner and S. Sidki, The generation of $\operatorname{GL}(n, \mathbf{Z})$ by finite state automata, Internat. J. Algebra Comput., 8 (1998), no. 1, 127-139. Zbl 0923.20023 MR 1492064

[12] J. W. Cannon, The combinatorial structure of cocompact discrete hyperbolic groups, Geom. Dedicata, 16 (1984), no. 2, 123-148. Zbl 0606.57003 MR 758901

[13] C. Champetier, Propriétés statistiques des groupes de présentation finie (French), Adv. Math., 116 (1995), no. 2, 197-262. Zbl 0847.20030 MR 1363765

[14] M. Coornaert and A. Papadopoulos, Symbolic dynamics and hyperbolic groups, Lecture Notes in Mathematics, 1539, Springer-Verlag, Berlin, 1993. Zbl 0783.58017 MR 1222644

[15] M. Coornaert and A. Papadopoulos, Horofunctions and symbolic dynamics on Gromov hyperbolic groups, Glasg. Math. J., 43 (2001), no. 3, 425-456. Zbl 1044.20027 MR 1878587

[16] M. Coornaert and A. Papadopoulos, Symbolic coding for the geodesic flow associated to a word hyperbolic group, Manuscripta Math., 109 (2002), no. 4, 465-492. Zbl 1045.20036 MR 1946714

[17] F. Dal'bo, M. Peigné, and A. Sambusetti, On the horoboundary and the geometry of rays of negatively curved manifolds, Pacific J. Math., 259 (2012), no. 1, 55-100. Zbl 1260.53068 MR 2988483

[18] M. Develin, Cayley compactifications of abelian groups, Ann. Comb., 6 (2002), no. 3-4, 295-312. Zbl 1021.05045 MR 1980341

[19] S. M. Gersten, Problems on automatic groups, in Algorithms and classification in combinatorial group theory (Berkeley, CA, 1989), 225-232, Math. Sci. Res. Inst. Publ., 23, Springer, New York, 1992. Zbl 0781.20022 MR 1230636

[20] E. Ghys and P. de la Harpe (eds.), Sur les groupes hyperboliques d'après Mikhael Gromov (Bern, 1988), Progr. Math., 83, Birkhäuser Boston, Boston, MA, 1990. Zbl 0731.20025 MR 1086657

[21] R. I. Grigorčuk, On Burnside's problem on periodic groups (Russian), Funktsional. Anal. i Prilozhen., 14 (1980), no. 1, 53-54. Zbl 0595.20029 MR 565099

[22] R. I. Grigorchuk, Solved and unsolved problems around one group, in Infinite groups: geometric, combinatorial and dynamical aspects, 117-218, Progr. Math., 248, Birkhäuser, Basel, 2005. Zbl 1165.20021 MR 2195454

[23] R. I. Grigorchuk, P. Linnell, T. Schick, and A. Żuk, On a question of Atiyah, C. R. Acad. Sci. Paris Sér. I Math., 331 (2000), no. 9, 663-668. Zbl 0969.57022 MR 1797748 
[24] R. I. Grigorchuk, V. Nekrashevych, and V. Sushchanskiĭ, Automata, dynamical systems, and groups, Proc. Steklov Inst. Math., (2000), no. 4(231), 128-203. Zbl 1155.37311 MR 1841755

[25] R. I. Grigorchuk and A. Żuk, The lamplighter group as a group generated by a 2state automaton, and its spectrum, Geom. Dedicata, 87 (2001), no. 1-3, 209-244. Zbl 0990.60049 MR 1866850

[26] M. Gromov, Hyperbolic groups, in Essays in group theory, 75-263, Math. Sci. Res. Inst. Publ., 8, Springer, New York, 1987. Zbl 0634.20015 MR 919829

[27] N. Gupta and S. Sidki, On the Burnside problem for periodic groups, Math. Z., 182 (1983), no. 3, 385-388. Zbl 0513.20024 MR 696534

[28] D. F. Holt, S. Rees, C. E. Röver, and R. M. Thomas, Groups with context-free coword problem, J. London Math. Soc. (2), 71 (2005), no. 3, 643-657. Zbl 1104.20033 MR 2132375

[29] L. Ji and A.-S. Schilling, Toric varieties vs. horofunction compactifications of polyhedral norms, Enseign. Math., 63 (2017), no. 3-4, 375-401. Zbl 1402.14065 MR 3852176

[30] I. Kapovich and N. Benakli, Boundaries of hyperbolic groups, in Combinatorial and geometric group theory (New York, 2000/Hoboken, NJ, 2001), 39-93, Contemp. Math., 296, Amer. Math. Soc., Providence, RI, 2002. Zbl 1044.20028 MR 1921706

[31] I. Kapovich and D. T. Wise, The equivalence of some residual properties of wordhyperbolic groups, J. Algebra, 223 (2000), no. 2, 562-583. Zbl 0951.20029 MR 1735163

[32] B. P. Kitchens, Symbolic dynamics. One-sided, two-sided and countable state Markov shifts, Universitext, Springer-Verlag, Berlin, 1998. Zbl 0892.58020 MR 1484730

[33] T. Klein and A. Nicas, The horofunction boundary of the Heisenberg group, Pacific J. Math., 242 (2009), no. 2, 299-310. Zbl 1189.53040 MR 2546714

[34] M. Laca, I. Raeburn, J. Ramagge, and M. F. Whittaker, Equilibrium states on operator algebras associated to self-similar actions of groupoids on graphs, Adv. Math., 331 (2018), 268-325. Zbl 1392.37007 MR 3804678

[35] J. Lehnert and P. Schweitzer, The co-word problem for the Higman-Thompson group is context-free, Bull. Lond. Math. Soc., 39 (2007), no. 2, 235-241. Zbl 1166.20025 MR 2323454

[36] I. G. Lysionok, Problem 22 of "Problem Session", in Geometric and Combinatorial Group Theory (Edinburgh, 1993), 322-325, LMS Lecture Notes Series, 204, Cambridge University Press, Cambridge, 1995.

[37] H. Miyachi, Extremal length boundary of the Teichmüller space contains non-Busemann points, Trans. Amer. Math. Soc., 366 (2014), no. 10, 5409-5430. Zbl 1318.32020 MR 3240928

[38] V. Nekrashevych, Self-similar groups, Mathematical Surveys and Monographs, 117, American Mathematical Society, Providence, RI, 2005. Zbl 1087.20032 MR 2162164

[39] V. Nekrashevych, Iterated monodromy groups, in Groups St Andrews 2009 in Bath. Volume 1, 41-93, London Math. Soc. Lecture Note Ser., 387, Cambridge Univ. Press, Cambridge, 2011. Zbl 1235.37016 MR 2858850 
[40] V. Nekrashevych and S. Sidki, Automorphisms of the binary tree: state-closed subgroups and dynamics of 1/2-endomorphisms, in Groups: topological, combinatorial and arithmetic aspects, 375-404, London Math. Soc. Lecture Note Ser., 311, Cambridge Univ. Press, Cambridge, 2004. Zbl 1144.20305 MR 2073355

[41] G. Niblo (ed.), Geometric group theory 1991 problem list, in Geometric group theory, Vol. 1 (Sussex, 1991), 208-212, London Math. Soc. Lecture Note Ser., 181, Cambridge Univ. Press, Cambridge, 1993. MR 1238528

[42] A. Yu. Ol'shanskiǔ, Almost every group is hyperbolic, Internat. J. Algebra Comput., 2 (1992), no. 1, 1-17. Zbl 0780.17025 MR 1167524

[43] C. Röver, Constructing finitely presented simple groups that contain Grigorchuk groups, J. Algebra, 220 (1999), no. 1, 284-313. Zbl 0940.20034 MR 1714140

[44] P. V. Silva and B. Steinberg, On a class of automata groups generalizing lamplighter groups, Internat. J. Algebra Comput., 15 (2005), no. 5-6, 1213-1234. Zbl 1106.20028 MR 2197829

[45] M. Vorobets and Y. Vorobets, On a free group of transformations defined by an automaton, Geom. Dedicata, 124 (2007), 237-249. Zbl 1183.20024 MR 2318547

[46] C. Walsh, The horofunction boundary of the Hilbert geometry, Adv. Geom., 8 (2008), no. 4, 503-529. Zbl 1155.53335 MR 2456635

[47] C. Walsh, The action of a nilpotent group on its horofunction boundary has finite orbits, Groups Geom. Dyn., 5 (2011), no. 1, 189-206. Zbl 1262.20045 MR 2763785

[48] C. Webster and A. Winchester, Boundaries of hyperbolic metric spaces, Pacific J. Math., 221 (2005), no. 1, 147-158. Zbl 1177.53042 MR 2194149

Received 14 October, 2019

J. Belk, School of Mathematics and Statistics, University of St Andrews,

St Andrews KY16 9SS, UK

E-mail: jmb42@st-andrews.ac.uk

C. Bleak, School of Mathematics and Statistics, University of St Andrews,

St Andrews KY16 9SS, UK

E-mail: collin.bleak@st-andrews.ac.uk

F. Matucci, Dipartimento di Matematica e Applicazioni,

Université degli Studi di Milano-Bicocca, Milan 20125, Italy

E-mail: francesco.matucci@unimib.it 